\title{
Wall-crossing formulas in Hamiltonian geometry
}

\author{
Paul-Emile PARADAN \\ Institut de Mathématiques et de Modèlisation de Montpellier (I3M) \\ CNRS : UMR5149, Université Montpellier II, France \\ paradan@math.univ-montp2.fr
}

Summary. In this article, we study the local invariants associated to the Hamiltonian action of a compact torus. Our main results are wall-crossing formulas between invariants attached to adjacent connected components of regular values of the moment map.

Key words: moment map, equivariant cohomology, geometric quantization, transversally elliptic.

\subsection{Introduction}

Let $(M, \Omega)$ be a compact symplectic manifold with the Hamiltonian action of a compact torus $T$, and moment map $\Phi: M \rightarrow \mathfrak{t}^{*}$. Let us assume that the action is effective. We are interested here in two global invariants:

1. the Duistermaat-Heckman measure $\mathrm{DH}(M)$ which is the pushforward by $\Phi$ of the Liouville volume form,

2. the Riemann-Roch characters $R R\left(M, L^{\otimes k}\right), k \geq 1$, which are virtual representations of $T$. Here the data $(M, \Omega, \Phi)$ is prequantized by a KostantSouriau line bundle $L$.

Let $\Lambda^{*} \subset \mathfrak{t}^{*}$ be the weight lattice of $T$. For every couple $(\mu, k) \in \Lambda^{*} \times \mathbb{Z}^{>0}$, we denote by $\mathrm{m}(\mu, k) \in \mathbb{Z}$ the multiplicity of the weight $\mu$ in $R R\left(M, L^{\otimes k}\right)$.

One stricking property of the moment map is that its image $\Phi(M)$ is a convex polytope in $\mathfrak{t}^{*}$. In fact, as noted for example in [17] or [20], each component of the set of regular values of $\Phi$ is either an open convex polytope contained in $\Phi(M)$, or the open subset $\mathfrak{c}_{\mathrm{ext}}=\mathfrak{t}^{*} \backslash \Phi(M)$.

Let us fix a connected component $\mathfrak{c}$ of regular values of $\Phi$. A celebrated Theorem of Duistermaat and Heckman [15] tells us that the measure $\mathrm{DH}(M)$ is equal to a polynomial $\mathrm{DH}_{\mathfrak{c}}$ times a Lebesgue measure on the open subset $\mathfrak{c}$. Note that $\mathrm{DH}_{\mathfrak{c}_{\text {ext }}}$ is the zero polynomial. 
The "quantization commutes with reduction" Theorem $[28,29]$ shows that there exists a periodic polynomial $\mathrm{m}_{\mathfrak{c}}: \Lambda^{*} \times \mathbb{Z} \rightarrow \mathbb{Z}$ which coincides with the multiplicity map $\mathrm{m}: \Lambda^{*} \times \mathbb{Z}^{>0} \rightarrow \mathbb{Z}$ on the cone of $\mathfrak{t}^{*} \times \mathbb{R}$ generated by $\mathfrak{c} \times\{1\}$. The periodic polynomial $\mathrm{m}_{\mathfrak{c}}$ is defined by a Kawasaki-Riemann-Roch formula on a symplectic quotient $\mathcal{M}_{a}=\Phi^{-1}(a) / T$ where $a \in \mathfrak{c}$. As a corollary, we get that $\mathrm{DH}_{\mathfrak{c}}$ is the semi-classical limit of $\mathrm{m}_{\mathfrak{c}}$ : one has

$$
\lim _{k \rightarrow \infty} \frac{\mathrm{m}_{\mathfrak{c}}(k \mu, k)}{k^{d}}=\frac{1}{(2 \pi)^{d}} \mathrm{DH}_{\mathfrak{c}}(\mu)
$$

for every $\mu \in \Lambda^{*}$. Here $d=\frac{1}{2} \operatorname{dim} \mathcal{M}_{a}$.

We have seen that the global invariants $\mathrm{DH}(M), R R\left(M, L^{\otimes k}\right), k \geq 1$ gives rises to a family of local invariants $\mathrm{DH}_{\mathfrak{c}}, \mathrm{m}_{\mathfrak{c}}$, where $\mathfrak{c}$ runs over the connected component of regular values of $\Phi$.

This paper is concerned by the differences $\mathrm{DH}_{\mathfrak{c}_{+}}-\mathrm{DH}_{\mathfrak{c}_{-}}$and $\mathrm{m}_{\mathfrak{c}_{+}}-\mathrm{m}_{\mathfrak{c}_{-}}$ when $\mathfrak{c}_{ \pm}$are two adjacent connected components of regular values of $\Phi$. Let $\Delta \subset \mathfrak{t}^{*}$ be the hyperplane that separates $\mathfrak{c}_{ \pm}$. Some continuity properties are known:

1. the polynomial $\mathrm{DH}_{\mathfrak{c}_{+}}-\mathrm{DH}_{\mathfrak{c}_{-}}$is divisible by a certain power of the equation the hyperplane $\Delta$ (see [17] and [12]),

2. the periodic polynomial $\mathrm{m}_{\mathfrak{c}_{+}}-\mathrm{m}_{\mathfrak{c}_{-}}$vanishes on

$$
\left\{(\mu, k) \in \Lambda^{*} \times \mathbb{Z} \mid \mu \in k \Delta\right\} .
$$

See [29].

In this paper, we compute explicitely the difference $\mathrm{DH}_{\mathfrak{c}_{+}}-\mathrm{DH}_{\mathfrak{c}_{-}}$, and we show that $\mathrm{m}_{\mathfrak{c}_{+}}-\mathrm{m}_{\mathfrak{c}_{-}}$vanishes also on some translates of (1.2).

Let us introduce some notations. We denote by $T_{\Delta} \subset T$ the subtorus of dimension 1 that has for Lie algebra the one dimensional subspace $\mathfrak{t}_{\Delta}$ which is orthogonal to the direction of $\Delta$. Let $\beta \in \mathfrak{t}_{\Delta}$ be the primitive element of the lattice $\operatorname{ker}(\exp : \mathfrak{t} \rightarrow T)$ which is pointing out of $\mathfrak{c}_{-}$.

We make the choice of a decomposition $T=T / T_{\Delta} \times T_{\Delta}$, where $T / T_{\Delta}$ denotes a subtorus de $T$. At the level of Lie algebras, we have then $\mathfrak{t}=$ $\left(\mathfrak{t} / \mathfrak{t}_{\Delta}\right) \oplus \mathfrak{t}_{\Delta}$ and $\mathfrak{t}^{*}=\left(\mathfrak{t} / \mathfrak{t}_{\Delta}\right)^{*} \oplus \mathfrak{t}_{\Delta}^{*}$ : hence $\xi+\left(\mathfrak{t} / \mathfrak{t}_{\Delta}\right)^{*}=\Delta$ for any $\xi \in \Delta$. We denote $\mathcal{S}(\mathfrak{t})$ the algebra of polynomials on the vector space $\mathfrak{t}^{*}$. We will consider the polynomial $\mathrm{DH}_{\mathfrak{c}_{+}}-\mathrm{DH}_{\mathfrak{c}_{-}} \in \mathcal{S}(\mathfrak{t})$ relatively to the decomposition

$$
\mathcal{S}(\mathfrak{t})=\bigoplus_{j \in \mathbb{N}} \mathcal{S}\left(\mathfrak{t} / \mathfrak{t}_{\Delta}\right) \beta^{j}
$$

Let us choose $\xi \in \Delta$ in the relative interior of $\overline{\mathfrak{c}_{+}} \cap \overline{\overline{c_{-}}}$in $\Delta$. We consider the family $\mathcal{F}$ of connected components $Z \subset M^{T_{\Delta}}$ such that $\xi \in \Phi(Z) \subset \Delta$. It is easy to see that $\mathcal{F}$ does not depend of the choice of $\xi$ : we have $\overline{\mathfrak{c}_{+}} \cap \overline{\mathfrak{c}_{-}} \subset \Phi(Z)$ for all $Z \in \mathcal{F}$. For each $Z \in \mathcal{F}$, we denote 


$$
\Phi_{Z}: Z \rightarrow\left(\mathfrak{t} / \mathfrak{t}_{\Delta}\right)^{*}
$$

the restriction of the map $\Phi-\xi$ to the symplectic sub-manifold $Z$. The map $\Phi_{Z}$ is a moment map relative to the Hamiltonian action of $T / T_{\Delta}$ on $Z$. Let $\mathrm{DH}(Z)$ be Duistermaat-Heckman measure on $\left(\mathfrak{t} / \mathfrak{t}_{\Delta}\right)^{*}$ associated to the moment map $\Phi_{Z}$. Since 0 is a regular value of $\Phi_{Z}$, we may consider the Duistermaat-Heckman polynomial

$$
\mathrm{DH}_{0}(Z) \in \mathcal{S}\left(\mathfrak{t} / \mathfrak{t}_{\Delta}\right)
$$

such that $\mathrm{DH}(Z)\left(a^{\prime}\right)=\mathrm{DH}_{0}(Z)\left(a^{\prime}\right) d a^{\prime}$ for $a^{\prime}$ in a neighborhood of 0 in $\left(\mathfrak{t} / \mathfrak{t}_{\Delta}\right)^{*}$.

For $Z \in \mathcal{F}$, we consider the symplectic reduction

$$
\mathcal{Z}_{\xi}=\Phi_{Z}^{-1}(0) /\left(T / T_{\Delta}\right),
$$

and the normal bundle $N_{Z}$ of $Z$ in $M$. Let $2 d_{Z}$ be the dimension of $\mathcal{Z}_{\xi}$ and $2 r_{Z}$ be the (real) rank of $N_{Z}$. We prove in Section 1.2 the following.

Theorem A. We have

$$
\left(\mathrm{DH}_{\mathfrak{c}_{+}}-\mathrm{DH}_{\mathfrak{c}_{-}}\right)(a)=\sum_{Z \in \mathcal{F}} \mathbf{D}_{Z}(a-\xi), \quad a \in \mathfrak{t}^{*}
$$

where each polynomial $\mathbf{D}_{Z} \in \mathcal{S}(\mathfrak{t})$ admits the following decomposition

$$
\mathbf{D}_{Z}=\frac{\beta^{r_{Z}-1}}{\operatorname{det}_{Z}^{1 / 2}\left(\frac{-\mathcal{L}_{\beta}}{2 \pi}\right)}\left(\frac{\mathrm{DH}_{0}(Z)}{\left(r_{Z}-1\right) !}+\sum_{k=1}^{d_{Z}} \beta^{k} \mathrm{Q}_{Z, k}\right) .
$$

Each polynomial $\mathrm{Q}_{Z, k}$ belongs to $\mathcal{S}\left(\mathfrak{t} / \mathfrak{t}_{\Delta}\right)$ and is of degree less than $d_{Z}-k$. The term $\operatorname{det}_{Z}^{1 / 2}\left(\frac{-\mathcal{L}_{\beta}}{2 \pi}\right) \in \mathbb{Z}$ is the Pfaffian of the infinitesimal action of $\frac{-\beta}{2 \pi}$ on the fibers of the normal bundle $N_{Z}$.

Theorem A generalizes previous results of Guillemin-Lerman-Sternberg [17] and Brion-Procesi [12]. In Section 1.2.4 we give the precise definition of the polynomials $\mathrm{Q}_{Z, k}$.

Suppose now that $M$ is prequantized by a Kostant-Souriau line bundle $L$. The hyperplane $\Delta$ is defined by the equation

$$
\frac{\langle a, \beta\rangle}{2 \pi}-r_{\Delta}=0, \quad a \in \mathfrak{t}^{*},
$$

for some $r_{\Delta} \in \mathbb{Z}$. The bundle $N_{Z}$ decomposes as the sum of two polarized sub-bundles $N_{Z}^{ \pm, \beta}$. Let $s_{Z}^{ \pm} \in \mathbb{N}$ be the absolute value of the trace of $\frac{1}{2 \pi} \mathcal{L}_{\beta}$ on $N_{Z}^{ \pm, \beta}$. Note that the integer $s_{Z}^{+}+s_{Z}^{-}$is larger than half of the codimension of $Z$ in $M$.

We prove in Section 1.3.5 the following 
Theorem B Let $s^{ \pm}:=\inf _{Z \in \mathcal{F}} s_{Z}^{ \pm}$. We have $\mathrm{m}_{\mathfrak{c}_{+}}(\mu, k)=\mathrm{m}_{\mathfrak{c}_{-}}(\mu, k)$ when

$$
-s^{-}<\frac{\langle\mu, \beta\rangle}{2 \pi}-k r_{\Delta}<s^{+} \text {. }
$$

Note that the symplectic orbifolds $\mathcal{Z}_{\xi}, Z \in \mathcal{F}$ are the connected component of the symplectic reduction

$$
\mathcal{M}_{\xi}^{\Delta}:=\left(\Phi^{-1}(\xi) \cap M^{T_{\Delta}}\right) /\left(T / T_{\Delta}\right) .
$$

We have the following refinement au Theorem B.

Theorem $\mathbf{C}$ If $\mathcal{M}_{\xi}^{\Delta}$ is connected, the inequalities (1.4) are optimal, i.e. there exists $(\mu, k)$ such that $\frac{\langle\mu, \beta\rangle}{2 \pi}-k r_{\Delta}= \pm s^{ \pm}$and $\mathrm{m}_{\mathfrak{c}_{+}}(\mu, k) \neq \mathrm{m}_{\mathfrak{c}_{-}}(\mu, k)$.

In Section 1.4 we apply Theorem $\mathbf{B}$ to the particular cases where $M$ is a integral coadjoint orbit of a compact Lie group $G$. In Section 1.4.4, we study more precisely the case $G=\mathrm{SU}(n)$ : here our result precises some of the results of Billey-Guillemin-Rassart [10].

In Section 1.5, we obtain a strong version of Theorem $\mathbf{B}$ in the case of an action of a torus $T$ on a complex vector spaces $\mathbb{C}^{d}$. The quantization of this action is in some sense the vector space $\operatorname{Pol}\left(\mathbb{C}^{d}\right)$ of complex polynomials on $\mathbb{C}^{d}$. The $T$-multiplicities of $\operatorname{Pol}\left(\mathbb{C}^{d}\right)$ are given by a partition function $N_{R}: \Lambda^{*} \rightarrow \mathbb{N}$. It was observed in $[13,35]$ that there exists a finite decomposition of the vector space $\mathfrak{t}^{*}$ in conic chambers such that $N_{R}$ is periodic polynomial on each piece.

Let $\mathfrak{c}_{ \pm}$be two adjacents chambers, and let $P_{\mathfrak{c} \pm}$ be the corresponding periodic polynomials computing $N_{R}$ on each chambers. The main result of Section 1.5 is the formula (1.109) which depicts the periodic polynomial $P_{\mathbf{c}_{+}}-P_{\mathfrak{c}_{-}}$as a convolution of distributions. Recently ${ }^{1}$, Boyal-Vergne [11] and De ConciniProcesi-Vergne [14] proposed differents proofs of this formula.

Acknowledgments. I am grateful to Michèle Vergne for bringing me the reference [10] to my attention, and for explaining me her work with András Szenes [36].

\section{Notations}

Throughout the paper $T$ will denote a compact, connected abelian Lie group, and $\mathfrak{t}$ its Lie algebra. The integral lattice $\Lambda \subset \mathfrak{t}$ is defined as the kernel of exp : $\mathfrak{t} \rightarrow T$, and the real weight lattice $\Lambda^{*} \subset \mathfrak{t}^{*}$ is defined by : $\Lambda^{*}:=\operatorname{hom}(\Lambda, 2 \pi \mathbb{Z})$. Every $\mu \in \Lambda^{*}$ defines a 1-dimensional $T$-representation, denoted by $\mathbb{C}_{\mu}$, where $t=\exp X$ acts by $t^{\mu}:=e^{i\langle\mu, X\rangle}$. We denote by $R(T)$ the ring of characters of finite-dimensional $T$-representations. We denote by $R^{-\infty}(T)$ the set of generalized characters of $T$. An element $\chi \in R^{-\infty}(T)$ is of

\footnotetext{
${ }^{1}$ Our present paper is a revised version of the preprint math.SG/0411306
} 
the form $\chi=\sum_{\mu \in \Lambda^{*}} a_{\mu} \mathbb{C}_{\mu}$, where $\mu \mapsto a_{\mu}, \Lambda^{*} \rightarrow \mathbb{Z}$ has at most polynomial growth.

The symplectic manifolds are oriented by their Liouville volume forms. If $\left(Z, o_{Z}\right)$ is an oriented submanifold of an oriented manifold $\left(M, o_{M}\right)$, we take on the fibers of the normal bundle $N$ of $Z$ in $M$, the orientation $o_{N}$ satisfying $o_{M}=o_{Z} \cdot o_{N}$.

\subsection{Duistermaat-Heckman measures}

Let $(M, \Omega)$ be a symplectic manifold of dimension $2 n$ equipped with an Hamiltonian action of a torus $T$, with Lie algebra $\mathfrak{t}$. The moment map $\Phi: M \rightarrow \mathfrak{t}^{*}$ satisfies the relations $\Omega\left(X_{M},-\right)+d\langle\Phi, X\rangle=0, X \in \mathfrak{t}$. We assume in this section that $\Phi$ is proper, and that the generic stabiliser $\Gamma_{M}$ of $T$ on $M$ is finite.

The Duistermaat-Heckman measure $\mathrm{DH}(M)$ is defined as the pushforward by $\Phi$ of the Liouville volume form $\frac{\Omega^{n}}{n !}$ on $M$. For every $f \in \mathcal{C}^{\infty}\left(\mathfrak{t}^{*}\right)$ with compact support one has $\int_{\mathfrak{t}^{*}} \operatorname{DH}(M)(a) f(a)=\int_{M} f(\Phi) \frac{\Omega^{n}}{n !}$. In other terms

$$
\operatorname{DH}(M)(a)=\int_{M} \delta(a-\Phi) \frac{\Omega^{n}}{n !}, \quad a \in \mathfrak{t}^{*} .
$$

We can define $\mathrm{DH}(M)$ in terms of equivariant forms as follows. Let $\mathcal{A}(M)$ be the space of differential forms on $M$ with complex coefficients. We denote by $\mathcal{A}_{\text {temp }}^{-\infty}(\mathfrak{t}, M)$ the space of tempered generalized functions over $\mathfrak{t}$ with values in $\mathcal{A}(M)$, and by $\mathcal{M}_{\text {temp }}^{-\infty}\left(\mathfrak{t}^{*}, M\right)$ the space of tempered distributions over $\mathfrak{t}^{*}$ with values in $\mathcal{A}(M)$. Let $\mathcal{F}: \mathcal{A}_{\text {temp }}^{-\infty}(\mathfrak{t}, M) \rightarrow \mathcal{M}_{\text {temp }}^{-\infty}\left(\mathfrak{t}^{*}, M\right)$ be the Fourier transform normalized by the condition that $\mathcal{F}\left(X \mapsto e^{i\langle\xi, X\rangle}\right)$ is equal to the Dirac distribution $a \mapsto \delta(a-\xi)$.

Let $\Omega_{\mathfrak{t}}(X)=\Omega-\langle\Phi, X\rangle$ be the equivariant symplectic form. We have then $\mathcal{F}\left(e^{-i \Omega_{\mathrm{t}}}\right)=e^{-i \Omega} \delta(a-\Phi)$ and so

$$
\mathrm{DH}(M)=(i)^{n} \int_{M} \mathcal{F}\left(e^{-i \Omega_{\mathrm{t}}}\right) .
$$

\subsubsection{Equivariant cohomology and localization}

We first recall the Cartan model of equivariant cohomology with polynomial coefficients and the extension to generalized coefficients defined by Kumar and Vergne [26]. We give after a brief account to the method of localization developped in [30, 31],

Let $M$ be a manifold provided with an action of a compact connected Lie group $K$ with Lie algebra $\mathfrak{k}$. Let $d: \mathcal{A}(M) \rightarrow \mathcal{A}(M)$ be the exterior differentiation. Let $\mathcal{A}_{c}(M)$ be the sub-algebra of compactly supported differential forms. If $V$ is a vector field on $M$ we denote by $c(V): \mathcal{A}(M) \rightarrow \mathcal{A}(M)$ the 
contraction by $V$. The action of $K$ on $M$ gives a morphism $X \rightarrow X_{M}$ from $\mathfrak{k}$ to the Lie algebra of vector fields on $M$.

We consider the space of $K$-equivariant maps $\mathfrak{k} \rightarrow \mathcal{A}(M), X \mapsto \eta(X)$, equipped with the derivation $(D \eta)(X):=\left(d-c\left(X_{M}\right)\right)(\eta(X)), X \in \mathfrak{k}$. Since $D^{2}=0$, one can define the cohomology space $\operatorname{ker} D / \operatorname{Im} D$. The Cartan model $[7,21]$ considers polynomial maps and the associated cohomology is denoted $\mathcal{H}_{K}^{*}(M)$. Kumar and Vergne [26] studied the cohomology spaces $\mathcal{H}_{K}^{ \pm \infty}(M)$ obtained by taking $\mathcal{C}^{ \pm \infty}$ maps. Recall the construction $\mathcal{H}_{K}^{-\infty}(M)$.

The space $\mathcal{C}^{-\infty}(\mathfrak{k}, \mathcal{A}(M))$ of generalized functions on $\mathfrak{k}$ with values in the space $\mathcal{A}(M)$ is, by definition, the space $\operatorname{Hom}\left(m_{c}(\mathfrak{k}), \mathcal{A}(M)\right)$ of continuous $\mathbb{C}$-linear maps from the space $m_{c}(\mathfrak{k})$ of smooth compactly supported densities on $\mathfrak{k}$ to the space $\mathcal{A}(M)$, both endowed with the $\mathcal{C}^{\infty}$-topologies. We define $\mathcal{A}_{K}^{-\infty}(M):=\mathcal{C}^{-\infty}(\mathfrak{k}, \mathcal{A}(M))^{K}$ as the space of $K$-equivariant $\mathcal{C}^{-\infty}$ maps from $\mathfrak{k}$ to $\mathcal{A}(M)$. The differential $D$ defined on $\mathcal{C}^{\infty}(\mathfrak{k}, \mathcal{A}(M))$ admits a natural extension to $\mathcal{C}^{-\infty}(\mathfrak{k}, \mathcal{A}(M))$ and $D^{2}=0$ on $\mathcal{A}_{K}^{-\infty}(M)$ [26]. The cohomology associated to $\left(\mathcal{A}_{K}^{-\infty}(M), D\right)$ is called the $K$-equivariant cohomology with generalized coefficients and is denoted by $\mathcal{H}_{K}^{-\infty}(M)$. The subspace $\mathcal{A}_{K, c}^{-\infty}(M):=\mathcal{C}^{-\infty}\left(\mathfrak{k}, \mathcal{A}_{c}(M)\right)^{K}$ is stable under the differential $D$, and we denote by $\mathcal{H}_{K, c}^{-\infty}(M)$ the associated cohomology. When $M$ is oriented, the integration over $M$ gives rise to a map $\int_{M}: \mathcal{H}_{K, c}^{-\infty}(M) \rightarrow \mathcal{C}^{-\infty}(\mathfrak{k})^{K}$.

Localization procedure. Let $\lambda$ be a $K$-invariant 1 -form on $M$ and let

$$
\Phi_{\lambda}: M \rightarrow \mathfrak{k}^{*}
$$

be the $K$-equivariant map defined by $\left\langle\Phi_{\lambda}(m), X\right\rangle=\lambda\left(X_{M}\right)_{m}$ : then $D \lambda(X)=$ $d \lambda-\left\langle\Phi_{\lambda}, X\right\rangle$. The localization procedure developped in [30,31] is based on the existence of an inverse $[D \lambda]^{-1}$ of the $K$-equivariant form $D \lambda$. It is an equivariantly closed element of $\mathcal{A}_{K}^{-\infty}\left(M-\Phi_{\lambda}^{-1}(0)\right)$ defined by the integral

$$
[D \lambda]^{-1}(X)=i \int_{0}^{\infty} e^{-i t D \lambda(X)} d t .
$$

An open subset $\mathcal{U} \subset M$ is called adapted to $\lambda$ if $\mathcal{U}$ is $K$-invariant and if $(\partial \mathcal{U}) \cap \Phi_{\lambda}^{-1}(0)=\emptyset$. In [31], we associate to an open subset $\mathcal{U}$ adapted to $\lambda$, the following equivariantly closed form with generalized coefficients

$$
\mathrm{P}_{\lambda}^{\mathcal{U}}=\chi^{\mathcal{U}}+d \chi^{\mathcal{U}}[D \lambda]^{-1} \lambda
$$

Here $\chi^{\mathcal{U}} \in \mathcal{C}^{\infty}(M)$ is a $K$-invariant function supported in $\mathcal{U}$ which is equal to 1 in a neighborhood of $\mathcal{U} \cap \Phi_{\lambda}^{-1}(0)$. The cohomology class defined by $\mathrm{P}_{\lambda}^{\mathcal{U}}$ in $\mathcal{H}_{K}^{-\infty}(M)$ does not depend of $\chi^{\mathcal{U}}$. In particular $\mathrm{P}_{\lambda}^{\mathcal{U}}=0$ in $\mathcal{H}_{K}^{-\infty}(M)$ if $\mathcal{U} \cap \Phi_{\lambda}^{-1}(0)=\emptyset$. If $\mathcal{U} \cap \Phi_{\lambda}^{-1}(0)$ is compact, we take $\chi^{\mathcal{U}}$ with compact support, then $\mathrm{P}_{\lambda}^{\mathcal{U}}$ defines a cohomology class in $\mathcal{H}_{K, c}^{-\infty}(M)$. 


\subsubsection{Localization of $\mathrm{DH}(M)$}

We come back to the situation of a Hamiltonian action of a torus $T$ on a symplectic manifold $(M, \omega)$. We need two auxilliary data : a $T$-invariant Riemannian metric on $M$ denoted $(\cdot, \cdot)_{M}$, and a scalar product $(\cdot, \cdot)$ on $\mathfrak{t}^{*}$ which induces an identification $\mathfrak{t}^{*} \simeq \mathfrak{t}$.

Let $\mathcal{H}$ be the Hamiltonian vector field of the function $\frac{-1}{2}\|\Phi\|^{2}: M \rightarrow \mathbb{R}:$ for $m \in M$ we have $\mathcal{H}_{m}=\left.(\Phi(m))_{M}\right|_{m}$. Then for every $\xi \in \mathfrak{t}^{*}$, the Hamiltonian vector field of $\frac{-1}{2}\|\Phi-\xi\|^{2}$ is $\mathcal{H}-\xi_{M}$, and we consider the following $T$-invariant 1 -form

$$
\lambda_{\xi}=\left(\mathcal{H}-\xi_{M}, \cdot\right)_{M}
$$

with corresponding map $\Phi_{\lambda_{\xi}}: M \rightarrow \mathfrak{t}^{*}$ (see (1.6)). Here $\Phi_{\lambda_{\xi}}^{-1}(0)$ coincides with the subset $\operatorname{Cr}\left(\|\Phi-\xi\|^{2}\right) \subset M$ of critical points of the function $\|\Phi-\xi\|^{2}$, and $m \in \operatorname{Cr}\left(\|\Phi-\xi\|^{2}\right)$ if and only if $(\Phi(m)-\xi)_{M}$ vanishes at $m[30,31]$.

Definition 1.2.1 Let $\mathrm{P}_{\xi} \in \mathcal{H}_{T, c}^{-\infty}(M)$ be the cohomology class defined by $\mathrm{P}_{\lambda_{\xi}}^{\mathcal{U}}$, where $\mathcal{U}$ is a $T$-invariant relatively compact neighborhood of $\Phi^{-1}(\xi)$ such that $\overline{\mathcal{U}} \cap \operatorname{Cr}\left(\|\Phi-\xi\|^{2}\right)=\Phi^{-1}(\xi)$.

The cohomology class $\mathrm{P}_{\xi}$ will be used to localized the DuitermaatHeckman measure. For every $\xi \in \mathfrak{t}^{*}$, we define the distribution $\mathrm{DH}_{\xi}(M)$ by

$$
\mathrm{DH}_{\xi}(M)=(i)^{n} \mathcal{F}\left(\int_{M} \mathrm{P}_{\xi} e^{-i \Omega_{\mathrm{t}}}\right) .
$$

Here we can put the Fourier transform outside the integral because $\mathrm{P}_{\xi}$ is compactly supported on $M$. For any $\xi \in \mathfrak{t}^{*}$, let $r_{\xi}>0$ be the smallest nonzero critical value of the function $\|\Phi-\xi\|^{2}$. As a particular case of Proposition 3.8 in [31], we have

Proposition 1.2.2 Let $\xi$ be any point in $\mathfrak{t}^{*}$. The following equality of distributions on $\mathfrak{t}^{*}$

$$
\mathrm{DH}(M)=\mathrm{DH}_{\xi}(M)
$$

holds in the open ball $B\left(\xi, r_{\xi}\right) \subset \mathfrak{t}^{*}$.

We will now use the last Proposition, first to recover the classical result of Duistermaat and Heckman [15] concerning the polynomial behaviour of $\mathrm{DH}(M)$ on the open subset of regular values of $\Phi$. After we determine the difference taken by $\mathrm{DH}(M)$ between two adjacent regions of regular values.

\subsubsection{Polynomial behaviour}

We recall now the computation of the cohomology class $\mathrm{P}_{\xi}$ when $\xi$ is a regular value of $\Phi$, that is given in [30] [Section 6] for the torus case (see [31] [Section 3.1] for the case of Hamiltonian action of a compact Lie group). First recall the following basic result which shows that $\xi \mapsto \mathrm{DH}_{\xi}(M)$ is locally constant on the open subset of regular values of $\Phi$. 
Lemma 1.2.3 ([33]) If $\xi$ and $\xi^{\prime}$ belong to the same connected component of regular values of $\Phi$, we have $\mathrm{P}_{\xi}=\mathrm{P}_{\xi^{\prime}}$ in $\mathcal{H}_{T, c}^{-\infty}(M)$.

If we combine Lemma 1.2.3 with Proposition 1.2.2, we see that for any connected component $\mathfrak{c}$ of regular values of $\Phi$, we have

$$
\operatorname{DH}(M)(a)=\operatorname{DH}_{\xi}(M)(a), \quad a \in \mathfrak{c},
$$

for any $\xi \in \mathfrak{c}$. We have to compute $\operatorname{DH}_{\xi}(M)$ when $\xi$ a regular value of $\Phi$.

We consider the $T$-principal bundle $\Phi^{-1}(\xi) \rightarrow \mathcal{M}_{\xi}:=\Phi^{-1}(\xi) / T$ with curvature form $\omega_{\xi} \in \mathcal{H}^{2}\left(\mathcal{M}_{\xi}\right) \otimes \mathfrak{t}$. The orbifold $\mathcal{M}_{\xi}$ carries a canonical symplectic 2 -form $\Omega_{\xi}$. We denote

$$
\operatorname{Kir}_{\xi}: \mathcal{H}_{T}^{\infty}(M) \rightarrow \mathcal{H}^{*}\left(\mathcal{M}_{\xi}\right)
$$

the Kirwan morphism. For any $\psi \in \mathcal{C}^{\infty}(\mathfrak{t})$ and $\eta \in \mathcal{H}_{T}^{\infty}(M)$ we have $\mathbf{K i r}_{\xi}(\eta \psi)=\mathbf{K i r}_{\xi}(\eta) \psi\left(\omega_{\xi}\right)$, where the characteristic class $\psi\left(\omega_{\xi}\right)$ is the value of the differential operator $e^{\omega_{\xi}\left(\left.\frac{\partial}{\partial X}\right|_{0}\right)}$ against $\psi$. After [31][Prop. 3.11], we know that the integral

$$
\int_{\mathfrak{t}} \int_{M} \mathrm{P}_{\xi}(X) \eta(X) \psi(X) d X
$$

is equal to

$$
\frac{(-2 i \pi)^{\operatorname{dim} T} \operatorname{vol}(T, d X)}{\left|\Gamma_{M}\right|} \int_{\mathcal{M}_{\xi}} \operatorname{Kir}_{\xi}(\eta) \psi\left(\omega_{\xi}\right)
$$

for every equivariant class $\eta \in \mathcal{H}_{T}^{\infty}(M)$. Here $\operatorname{vol}(T, d X)$ is the volume of $T$ for the Haar mesure compatible with $d X$, and $\left|\Gamma_{M}\right|$ is the cardinal of $\Gamma_{M}$ (Note that the generic stabilizer of $T$ on $\Phi^{-1}(\xi)$ is $\Gamma_{M}$ ). In other words, for every $\eta \in \mathcal{H}_{T}^{\infty}(M)$ we have the following equality of generalized functions on $\mathfrak{t}^{*}$ supported at 0

$$
\int_{M} \mathrm{P}_{\xi}(X) \eta(X)=\frac{(-2 i \pi)^{\operatorname{dim} T}}{\left|\Gamma_{M}\right|} \int_{\mathcal{M}_{\xi}} \operatorname{Kir}_{\xi}(\eta) e^{\omega_{\xi}\left(\frac{\partial}{\partial X} \mid 0\right)} \operatorname{vol}(T,-) .
$$

For $\eta=e^{-i \Omega_{\mathrm{t}}}$ we have $\operatorname{Kir}_{\xi}(\eta)=e^{-i\left(\Omega_{\xi}-\left\langle\xi, \omega_{\xi}\right\rangle\right)}$, and a small computation shows that

$$
\mathcal{F}\left(e^{\omega_{\xi}\left(\left.\frac{\partial}{\partial X}\right|_{0}\right)} \operatorname{vol}(T,-)\right)(a)=e^{-i\left\langle a, \omega_{\xi}\right\rangle} \frac{d a}{(2 \pi)^{\operatorname{dim} T}}, \quad a \in \mathfrak{t}^{*} .
$$

where $d a$ is the Lebesgue measure on $\mathfrak{t}^{*}$ normalized by the condition: $\operatorname{vol}(T, d X)=$ 1 for the Lebesgue measure $d X$ on $\mathfrak{t}$ which is dual to $d a$.

Finally (1.10), (1.12) and (1.13) give

$$
\begin{aligned}
\operatorname{DH}_{\xi}(M)(a) & =\frac{(i)^{d}}{\left|\Gamma_{M}\right|} \int_{\mathcal{M}_{\xi}} e^{-i\left(\Omega_{\xi}+\left\langle a-\xi, \omega_{\xi}\right\rangle\right)} d a \\
& =\frac{1}{\left|\Gamma_{M}\right|} \int_{\mathcal{M}_{\xi}} \frac{\left(\Omega_{\xi}+\left\langle a-\xi, \omega_{\xi}\right\rangle\right)^{d}}{d !} d a,
\end{aligned}
$$

where $2 d=\operatorname{dim} \mathcal{M}_{\xi}$. 
Definition 1.2.4 For any connected component $\mathfrak{c}$ of regular values of $\Phi$ we denote $\mathrm{DH}_{\mathfrak{c}}$ the polynomial function $a \mapsto \frac{1}{\left|\Gamma_{M}\right|} \int_{\mathcal{M}_{\xi}} \frac{\left(\Omega_{\xi}+\left\langle a-\xi, \omega_{\xi}\right\rangle\right)^{d}}{d !}$, where $\xi$ is any point of $\mathfrak{c}$.

With the help of Proposition 1.2.2 we recover the classical result of Duistermaat and Heckman [15] that says that the measure $\mathrm{DH}(M)$ is locally polynomial $^{2}$ on the open subset of regular values of $\Phi$, and it's value at a regular element $\xi$ is equal to the symplectic volume of the reduce space $\mathcal{M}_{\xi}$ (times $\left|\Gamma_{M}\right|^{-1}$ ) . More precisely we have shown that for a connected component $\mathfrak{c}$ of regular values of $\Phi$ we have

$$
\operatorname{DH}(M)(a)=\operatorname{DH}_{\mathfrak{c}}(a) d a, \quad a \in \mathfrak{c} .
$$

\subsubsection{Wall-crossing formulas}

Consider now two connected regions $\mathfrak{c}_{ \pm}$of regular values of $\Phi$ separated by an hyperplane $\Delta \subset \mathfrak{t}^{*}$. In this section we compute the polynomial $\mathrm{DH}_{\mathfrak{c}_{+}}-\mathrm{DH}_{\mathfrak{c}_{-}}$. It generalizes previous results of Guillemin-Lerman-Sternberg [17] and BrionProcesi [12].

Let $\xi_{+}, \xi_{-}$be respectively two elements of $\mathfrak{c}_{+}$and $\mathfrak{c}_{-}$. We know from (1.2.2), (1.14) and Definition (1.2.4) that

$$
\left(\mathrm{DH}_{\mathfrak{c}_{+}}-\mathrm{DH}_{\mathfrak{c}_{-}}\right)(a) d a=(i)^{n} \mathcal{F}\left(\int_{M}\left(\mathrm{P}_{\xi_{+}}-\mathrm{P}_{\xi_{-}}\right) e^{-i \Omega_{\mathfrak{t}}}\right)(a), \quad a \in \mathfrak{t}^{*} .
$$

We recall now the computation of the cohomology class $\mathrm{P}_{\xi_{+}}-\mathrm{P}_{\xi_{-}} \in$ $\mathcal{H}_{T, c}^{-\infty}(M)$ done in [33]. We use the notation defined in the introduction.

Definition 1.2.5 We denote $M^{\Delta}$ the union of the connected component $Z$ of the fixed point set $M^{T \Delta}$ for which we have $\Phi(Z) \subset \Delta$. Let $M_{o}^{\Delta}$ be the $T$-invariant open subset of $M^{\Delta}$ where $T / T_{\Delta}$ acts locally freely.

For a connected component $Z \subset M^{\Delta}$, one has either $\overline{\mathfrak{c}_{+}} \cap \overline{\mathfrak{c}_{-}} \subset \Phi(Z)$ or $\overline{\mathfrak{c}_{+}} \cap \overline{\mathfrak{c}_{-}} \cap \Phi(Z)=\emptyset$. It is due to the fact that for any $\xi$ in relative interior of $\overline{\mathfrak{c}_{+}} \cap \overline{\mathfrak{c}_{-}}$in $\Delta$, and any $m \in \Phi^{-1}(\xi)$ the stabilizer $\mathfrak{t}_{m} \subset \mathfrak{t}$ is either equal to $\mathfrak{t}_{\Delta}$ or reduced to $\{0\}$.

The symplectic manifold $M^{\Delta}$ carries a Hamiltonian action of $T / T_{\Delta}$ with moment map $\left.\Phi\right|_{M^{\Delta}}: M^{\Delta} \rightarrow \Delta$ equal to the restriction of $\Phi$ on $M^{\Delta}$.

Let $\xi$ be a point in the relative interior of $\overline{\mathfrak{c}_{+}} \cap \overline{\mathfrak{c}_{-}}$in $\Delta$. From the previous discussion, we knows that $\xi$ is a regular value of $\left.\Phi\right|_{M \Delta}$, i.e. $\Phi^{-1}(\xi) \cap M^{T \Delta}$ is a submanifold of $M_{o}^{\Delta}$. Following Definition 1.2.1 we associate to $\xi$ the cohomology class

$$
\mathrm{P}_{\xi}^{\Delta} \in \mathcal{H}_{T / T_{\Delta}, c}^{-\infty}\left(M_{o}^{\Delta}\right) .
$$

${ }^{2}$ It is a polynomial times a Lebesgue measure on $\mathfrak{t}^{*}$. 
Let $\mathcal{H}^{*}\left(M_{o}^{\Delta}\right)^{\text {bas }}$ be the sub-algebra of $\mathcal{H}^{*}\left(M_{o}^{\Delta}\right)$ formed by the $T$-basic elements. Since the $T_{\Delta}$-action on $M_{o}^{\Delta}$ is trivial we have a canonical product operation

$$
\mathcal{H}_{T / T_{\Delta}, c}^{-\infty}\left(M_{o}^{\Delta}\right) \times \mathcal{C}^{-\infty}\left(\mathfrak{t}_{\Delta}, \mathcal{H}^{*}\left(M_{o}^{\Delta}\right)^{\text {bas }}\right) \stackrel{\wedge}{\longrightarrow} \mathcal{H}_{T, c}^{-\infty}\left(M_{o}^{\Delta}\right) .
$$

Proposition 1.2.6 ([33]) There exists a generalized function supported at 0 , $\delta^{\Delta} \in \mathcal{C}^{-\infty}\left(\mathfrak{t}_{\Delta}, \mathcal{H}^{*}\left(M_{o}^{\Delta}\right)^{\text {bas }}\right)$, such that

$$
\mathrm{P}_{\xi^{+}}-\mathrm{P}_{\xi^{-}}=\left(i_{\Delta}\right)_{*}\left(\mathrm{P}_{\xi}^{\Delta} \wedge \delta^{\Delta}\right) \quad \text { in } \quad \mathcal{H}_{T, c}^{-\infty}(M) .
$$

Here $\left(i_{\Delta}\right)_{*}: \mathcal{H}_{T, c}^{-\infty}\left(M_{o}^{\Delta}\right) \rightarrow \mathcal{H}_{T, c}^{-\infty}(M)$ is the direct image map relative to the inclusion $i_{\Delta}: M_{o}^{\Delta} \hookrightarrow M$.

We will now give the precise definition of $\delta^{\Delta}$. The decomposition $T=T_{\Delta} \times$ $T / T_{\Delta}$ and the trivial action of $T_{\Delta}$ on $M_{o}^{\Delta}$ determine a canonical isomorphism

$$
j_{\Delta}: \mathcal{H}_{T}^{*}\left(M_{o}^{\Delta}\right) \stackrel{\sim}{\longrightarrow} \mathcal{S}\left(\mathfrak{t}_{\Delta}^{*}\right) \otimes \mathcal{H}_{T / T_{\Delta}}^{*}\left(M_{o}^{\Delta}\right),
$$

where $\mathcal{S}\left(\mathfrak{t}_{\Delta}^{*}\right)$ is the algebra of complex polynomial functions on $\mathfrak{t}_{\Delta}$. Since the $T / T_{\Delta}$-action on $M_{o}^{\Delta}$ is locally free, we have the Chern-Weil isomorphism

$$
\operatorname{cv}_{\Delta}: \mathcal{H}_{T / T_{\Delta}}^{*}\left(M_{o}^{\Delta}\right) \stackrel{\sim}{\longrightarrow} \mathcal{H}^{*}\left(M_{o}^{\Delta}\right)^{\text {bas }} .
$$

Let $N_{\Delta}$ be the $T$-equivariant normal bundle of $M^{\Delta}$ in $M$, and let

$$
\operatorname{Eul}\left(N_{\Delta}\right) \in \mathcal{H}_{T}^{*}\left(M^{\Delta}\right)
$$

be the $T$-equivariant Euler class of $N_{\Delta}$. Now we consider the restriction of $\operatorname{Eul}\left(N_{\Delta}\right)$ on the open subset $M_{o}^{\Delta} \subset M^{\Delta}$, that we look through the isomorphism $\mathbf{c v}_{\Delta} \circ j_{\Delta}$ as an element of $\mathcal{S}\left(\mathfrak{t}_{\Delta}^{*}\right) \otimes \mathcal{H}^{*}\left(M_{o}^{\Delta}\right)^{\text {bas }}$ (for simplicity we keep the same notations $\operatorname{Eul}\left(N_{\Delta}\right)$ for this element). Following [30], we define inverses $\operatorname{Eul}_{ \pm \beta}^{-1}\left(N_{\Delta}\right) \in \mathcal{C}^{-\infty}\left(\mathfrak{t}_{\Delta}, \mathcal{H}^{*}\left(M_{o}^{\Delta}\right)^{\text {bas }}\right)$ by

$$
\operatorname{Eul}_{ \pm \beta}^{-1}\left(N_{\Delta}\right)(X)=\lim _{s \rightarrow+\infty} \frac{1}{\operatorname{Eul}\left(N_{\Delta}\right)(X \pm i s \beta)} .
$$

Here $\beta \in \mathfrak{t}_{\Delta}$ is chosen so that $\left\langle\xi^{+}-\xi^{-}, \beta\right\rangle>0$.

Definition 1.2.7 The generalized function $\delta^{\Delta} \in \mathcal{C}^{-\infty}\left(\mathfrak{t}_{\Delta}, \mathcal{H}^{*}\left(M_{o}^{\Delta}\right)^{\text {bas }}\right)$ is defined by

$$
\delta^{\Delta}:=\operatorname{Eul}_{\beta}^{-1}\left(N_{\Delta}\right)-\operatorname{Eul}_{-\beta}^{-1}\left(N_{\Delta}\right) .
$$

Since the polynomial $\operatorname{Eul}\left(N_{\Delta}\right)$ is invertible in a smooth manner on $\mathfrak{t}_{\Delta} \backslash\{0\}$ the generalized function $\delta^{\Delta}$ is supported at 0 .

Let $\xi$ be a point in the relative interior of $\overline{\mathfrak{c}_{+}} \cap \overline{\mathfrak{c}_{-}}$in $\Delta$. We consider the symplectic reduction 


$$
\mathcal{M}_{\xi}^{\Delta}:=\left(M^{\Delta} \cap \Phi^{-1}(\xi)\right) /\left(T / T_{\Delta}\right)
$$

If we restrict $\delta^{\Delta}$ to the submanifold $M^{\Delta} \cap \Phi^{-1}(\xi)$ we get the generalized function

$$
\delta_{\xi}^{\Delta} \in \mathcal{C}^{-\infty}\left(\mathfrak{t}_{\Delta}, \mathcal{H}^{*}\left(\mathcal{M}_{\xi}^{\Delta}\right)\right)
$$

Now we are able to compute the right hand side of (1.16). Let $\omega_{\xi}^{\Delta} \in$ $\mathcal{H}^{2}\left(\mathcal{M}_{\xi}^{\Delta}\right) \otimes \mathfrak{t} / \mathfrak{t}_{\Delta}$ be the curvature of the $T / T_{\Delta}$-principal bundle $M^{\Delta} \cap \Phi^{-1}(\xi) \rightarrow$ $\mathcal{M}_{\xi}^{\Delta}$. Let $\left|S_{\xi}^{\Delta}\right|$ be locally constant function on $M^{\Delta} \cap \Phi^{-1}(\xi)$ which is equal to the cardinal of the generic stabilizer of $T / T_{\Delta}$. From (1.12) and Proposition 1.2.6 we have

$$
\begin{aligned}
& \int_{M}\left(\mathrm{P}_{\xi_{+}}-\mathrm{P}_{\xi_{-}}\right)(X) e^{-i \Omega_{\mathrm{t}}(X)} \\
& =\int_{M_{o}^{\Delta}} \mathrm{P}_{\xi}^{\Delta}\left(X^{\prime}\right) \delta^{\Delta}\left(X^{\prime \prime}\right) e^{-i \Omega_{\mathfrak{t}}\left(X^{\prime}+X^{\prime \prime}\right)} \\
& =\frac{(-2 i \pi)^{\operatorname{dim} T-1}}{\left|S_{\xi}^{\Delta}\right|} \int_{\mathcal{M}_{\xi}^{\Delta}} e^{\omega_{\xi}^{\Delta}\left(\left.\frac{\partial}{\partial X^{\prime}}\right|_{0}\right)} \operatorname{vol}\left(T / T_{\Delta},-\right) \mathbf{K i r}_{\xi}^{\Delta}\left(e^{-i \Omega_{\mathfrak{t}}}\right)\left(X^{\prime \prime}\right) \delta_{\xi}^{\Delta}\left(X^{\prime \prime} \cdot 20\right)
\end{aligned}
$$

In the last equation the notations are the following :

1. $X=X^{\prime}+X^{\prime \prime}$ with $X^{\prime} \in \mathfrak{t} / \mathfrak{t}_{\Delta}$ and $X^{\prime \prime} \in \mathfrak{t}_{\Delta}$,

2. the Kirwan map $\operatorname{Kir}_{\xi}^{\Delta}: \mathcal{H}_{T}^{\infty}(M) \rightarrow \mathcal{C}^{\infty}\left(\mathfrak{t}_{\Delta}, \mathcal{H}^{*}\left(\mathcal{M}_{\xi}^{\Delta}\right)\right)$ is the composition of the restriction $\mathcal{H}_{T}^{\infty}(M) \rightarrow \mathcal{H}_{T}^{\infty}\left(M^{\Delta} \cap \Phi^{-1}(\xi)\right)$ with the Chern-Weil isomorphism $\mathcal{H}_{T}^{\infty}\left(M^{\Delta} \cap \Phi^{-1}(\xi)\right) \stackrel{\sim}{\longrightarrow} \mathcal{C}^{\infty}\left(\mathfrak{t}_{\Delta}, \mathcal{H}^{*}\left(\mathcal{M}_{\xi}^{\Delta}\right)\right)$.

A direct computation gives that $\operatorname{Kir}_{\xi}^{\Delta}\left(\Omega_{\mathfrak{t}}\right)\left(X^{\prime \prime}\right)=\Omega_{\xi}^{\Delta}-\left\langle\xi, \omega_{\xi}^{\Delta}+X^{\prime \prime}\right\rangle$ where $\Omega_{\xi}^{\Delta}$ is the induced symplectic form on the reduced space $\mathcal{M}_{\xi}^{\Delta}$. If we take the Fourier transform in (1.20) we get

$$
\begin{aligned}
& \left(\mathrm{DH}_{\mathfrak{c}_{+}}-\mathrm{DH}_{\mathfrak{c}_{-}}\right)(a) d a \\
& =\frac{(i)^{n+1-\operatorname{dim} T}}{\left|S_{\xi}^{\Delta}\right|}\left(\int_{\mathcal{M}_{\xi}^{\Delta}} e^{-i\left(\Omega_{\xi}^{\Delta}+\left\langle a^{\prime}, \omega_{\xi}^{\Delta}\right\rangle\right)} d a^{\prime} \mathcal{F}_{\mathfrak{t}_{\Delta}}\left(\delta_{\xi}^{\Delta}\right)\left(a^{\prime \prime}\right)\right)(a-\xi), \\
& =\sum_{Z \in \mathcal{F}} \frac{(i)^{n+1-\operatorname{dim} T}}{\left|S_{\xi}^{Z}\right|}\left(\int_{\mathcal{Z}_{\xi}} e^{-i\left(\Omega_{\xi}^{Z}+\left\langle a^{\prime}, \omega_{\xi}^{Z}\right\rangle\right)} d a^{\prime} \mathcal{F}_{\mathfrak{t}_{\Delta}}\left(\delta_{\xi}^{Z}\right)\left(a^{\prime \prime}\right)\right)(a-\xi \gamma 1.21)
\end{aligned}
$$

where $a=a^{\prime}+a^{\prime \prime}$ with $a^{\prime} \in\left(\mathfrak{t} / \mathfrak{t}_{\Delta}\right)^{*}$ and $a^{\prime \prime} \in\left(\mathfrak{t}_{\Delta}\right)^{*}$. In $(1.21)$, we write $\int_{\mathcal{M}_{\xi}^{\Delta}}=$ $\sum_{Z \in \mathcal{F}} \int_{\mathcal{Z}_{\xi}}$ where the sum is taken over the set $\mathcal{F}$ of connected components $Z$ of $M^{\Delta}$ that intersects $\Phi^{-1}(\xi)$ : we take then

$$
\mathcal{Z}_{\xi}=\left(Z \cap \Phi^{-1}(\xi)\right) /\left(T / T_{\Delta}\right)
$$

The 2-forms $\Omega_{\xi}^{\Delta}, \omega_{\xi}^{\Delta}$, the generic stabiliser $S_{\xi}^{\Delta}$, the vector bundle $N_{\Delta}$, the generalized function $\delta_{\xi}^{\Delta}$ restrict to each component $Z$ : we denote them respectively $\Omega_{\xi}^{Z}, \omega_{\xi}^{Z}, S_{\xi}^{Z}, N_{Z}, \delta_{\xi}^{Z}$. 
We recall now the computation of the Fourier tranform of the inverses $\operatorname{Eul}_{ \pm \beta}^{-1}\left(N_{Z}\right):=\left.\operatorname{Eul}_{ \pm \beta}^{-1}\left(N_{\Delta}\right)\right|_{Z}$ that is given in [30][Proposition 4.8.]. We consider a $T$-invariant scalar product on the fibers of the bundle $N_{\Delta}$. Let $R \in$ $\mathcal{A}^{2}\left(M_{o}^{\Delta}, \operatorname{so}\left(N_{\Delta}\right)\right)^{\text {bas }}$ be the curvature of a $T$-invariant and $T / T_{\Delta}$-horizontal Euclidean connexion on $N_{\Delta}$ : we denote by $R^{Z} \in \mathcal{A}^{2}\left(Z, \operatorname{so}\left(N_{Z}\right)\right)^{\text {bas }}$ the restriction of $R$ to a component $Z \in \mathcal{F}$. The curvature commutes with the infinitesimal action $\mathcal{L}_{X}$ of $X \in \mathfrak{t}_{\Delta}$, and with the complex structure $J_{\beta}=\mathcal{L}_{\beta}\left(-\mathcal{L}_{\beta}^{2}\right)^{1 / 2}$ on $N_{\Delta}$ defined by $\beta \in \mathfrak{t}_{\Delta}$.

We denote by $S^{\bullet}$ the symmetric algebra of the complex vector bundle $\left(N_{\Delta}, J_{\beta}\right)$. We keep the same notation for the restriction of $S^{\bullet}$ on the submanifolds $Z, \Phi^{-1}(\xi) \cap M^{\Delta}$, and for the induced orbifold vector bundle on the reduced spaces $\mathcal{Z}_{\xi}$ and $\mathcal{M}_{\xi}^{\Delta}$. For each $k \in \mathbb{N}$, we denote by $\operatorname{Tr}_{S^{k}}$ the trace operator defined on the complex endomorphisms of $S^{k}$. For a complex endomorphism $A$ of $N_{\Delta}$, we denote by $A^{\otimes k}$ the induced endomorphism on $S^{k}$. For any $X \in \mathfrak{t}_{\Delta}$, the complex endomorphism $\mathcal{L}_{X}^{-1} R^{Z}$ is symmetric. Hence the trace $\operatorname{Tr}_{S^{k}}\left(\left(\mathcal{L}_{X}^{-1} R^{Z}\right)^{\otimes k}\right)$ is a basic real differential form of degree $2 k$ on $Z$ which does not depend of the choice of complex structures $\left(J_{\beta}\right.$ or $\left.J_{-\beta}\right)$.

Let $\beta^{*} \in \mathfrak{t}_{\Delta}^{*}$ the dual of $\beta \in \mathfrak{t}_{\Delta}$.

Proposition 1.2.8 ([30]) For a smooth function $f$ on $\mathfrak{t}_{\Delta}^{*}$ with compact support we have $\int_{\mathfrak{t}^{*}} \mathcal{F}_{\mathfrak{t}_{\Delta}}\left(\operatorname{Eul}_{\beta}^{-1}\left(N_{Z}\right)\right)\left(a^{\prime \prime}\right) f\left(a^{\prime \prime}\right)=\int_{0}^{\infty} P_{Z}(t) f\left(t \beta^{*}\right) d t$ where $P_{Z}$ is the polynomial on $\mathbb{R}$ defined by:

$P_{Z}(t)=\frac{(2 \pi i)^{r_{Z}}}{\operatorname{det}_{Z}^{1 / 2}\left(\mathcal{L}_{\beta}\right)}\left(\frac{t^{r_{Z}-1}}{\left(r_{Z}-1\right) !}+\sum_{k=1}^{\operatorname{dim}(Z) / 2}(i)^{k} \operatorname{Tr}_{S^{k}}\left(\left(\mathcal{L}_{\beta}^{-1} R^{Z}\right)^{\otimes k}\right) \frac{t^{r_{Z}-1+k}}{\left(r_{Z}-1+k\right) !}\right)$.

Here $\operatorname{det}_{Z}^{1 / 2}\left(\mathcal{L}_{\beta}\right)$ is the Pfaffian of $\mathcal{L}_{\beta}$ on $N_{Z}$, and $r_{Z}=\operatorname{rk}_{\mathbb{C}}\left(N_{Z}\right)$.

One checks then that

$$
\begin{aligned}
\int_{\mathfrak{t}_{\Delta}^{*}} \mathcal{F}_{\mathfrak{t}_{\Delta}}\left(\operatorname{Eul}_{-\beta}^{-1}\left(N_{Z}\right)\right)\left(a^{\prime \prime}\right) f\left(a^{\prime \prime}\right) & =\int_{0}^{\infty}-P_{Z}(-t) f\left(-t \beta^{*}\right) d t \\
& =-\int_{-\infty}^{0} P_{Z}(t) f\left(t \beta^{*}\right) d t
\end{aligned}
$$

Hence the distribution $\mathcal{F}_{\mathfrak{t}_{\Delta}}\left(\delta^{Z}\right)$ is equal to $P_{Z}(\beta) d \beta$. From now one we fix $\beta$ as the primitive element of $\mathfrak{t}_{\Delta} \cap \Lambda$ which point out $\mathfrak{c}_{-}$. Then $d \beta$ and $d \beta^{*}$ are then (dual) Lebesgue measure on $\mathfrak{t}^{*}$ and $\mathfrak{t}$ : we have $\operatorname{vol}\left(T_{\Delta}, d \beta^{*}\right)=1$.

Let $R_{\xi}^{Z}$ be the restriction of the curvature $R^{Z}$ to the submanifold $Z \cap$ $\Phi^{-1}(\xi)$. Since $R^{Z}$ is $T / T_{\Delta}$-basic, $\operatorname{Tr}_{S^{k}}\left(\left(\mathcal{L}_{\beta}^{-1} R_{\xi}^{Z}\right)^{\otimes k}\right)$ can be seen as a real differential form of degree $2 k$ on the orbifold $\mathcal{Z}_{\xi}=\left(Z \cap \Phi^{-1}(\xi)\right) /\left(T / T_{\Delta}\right)$.

Each connected component $Z$ of $M^{\Delta}$ is a $T / T_{\Delta}$ Hamiltonian manifold: we take for moment map $\Phi_{Z}: Z \rightarrow\left(\mathfrak{t} / \mathfrak{t}_{\Delta}\right)^{*}$ the restriction of $\Phi-\xi$ to $Z$. Hence 0 is a regular value of $\Phi_{Z}$. Let $\mathrm{DH}_{0}(Z)$ be the polynomial function on 
$\left(\mathfrak{t} / \mathfrak{t}_{\Delta}\right)^{*}=\left\{a \in \mathfrak{t}^{*} \mid\langle\beta, a\rangle=0\right\}$ such that $\mathrm{DH}(Z)\left(a^{\prime}\right)=\operatorname{DH}_{0}(Z)\left(a^{\prime}\right) d a^{\prime}$ near 0. Finally (1.21) together with the proposition 1.2 .8 give the following

Theorem 1.2.9 We have $\left(\mathrm{DH}_{\mathfrak{c}_{+}}-\mathrm{DH}_{\mathfrak{c}_{-}}\right)(a)=\sum_{Z \in \mathcal{F}} \mathbf{D}_{Z}(a-\xi), a \in \mathfrak{t}^{*}$ where each polynomial $\mathbf{D}_{Z} \in \mathcal{S}(\mathfrak{t})$ admits the following decomposition

$$
\mathbf{D}_{Z}=\frac{\beta^{r_{Z}-1}}{\operatorname{det}_{Z}^{1 / 2}\left(\frac{-\mathcal{L}_{\beta}}{2 \pi}\right)}\left(\frac{\mathrm{DH}_{0}(Z)}{\left(r_{Z}-1\right) !}+\sum_{k=1}^{d_{Z}} \beta^{k} \mathrm{Q}_{Z, k}\right)
$$

The polynomials $\mathrm{Q}_{Z, k} \in \mathcal{S}\left(\mathfrak{t} / \mathfrak{t}_{\Delta}\right)$ are defined by

$$
\mathrm{Q}_{Z, k}\left(a^{\prime}\right)=\frac{(-1)^{k}}{\left(r_{Z}-1+k\right) !\left|S_{\xi}^{Z}\right|} \int_{\mathcal{Z}_{\xi}} \frac{\left(\Omega_{\xi}^{Z}+\left\langle a^{\prime}, \omega_{\xi}^{Z}\right\rangle\right)^{d_{Z}-k}}{\left(d_{Z}-k\right) !} \operatorname{Tr}_{S^{k}}\left(\left(\mathcal{L}_{\beta}^{-1} R_{\xi}^{Z}\right)^{\otimes k}\right) .
$$

Here $2 d_{Z}=\operatorname{dim} \mathcal{Z}_{\xi}$ and $2 r_{Z}=\operatorname{dim} M-\operatorname{dim} Z$.

Remark 1.2.10 - The polynomial $\mathrm{DH}_{\mathfrak{c}_{+}}-\mathrm{DH}_{\mathfrak{c}_{-}}$is divisible by the factor $a \mapsto\langle a-\xi, \beta\rangle^{r-1}$ with $r=\inf _{Z \in \mathcal{F}} r_{Z}$. If $\Delta \cap \Phi(M)$ is not a facet of the polytope $\Phi(M)$ we have $r_{Z} \geq 2$ for all connected component $Z \in \mathcal{F}$, hence $r-1 \geq 1$.

- Suppose now that $\mathfrak{c}_{-}$is a connected component of regular values of $\Phi$ bording a facet $\Phi(M) \cap \Delta$ of the polytope $\Phi(M)$. Here $Z=\Phi^{-1}(\Delta)$ is a connected component of the fixed point set $M^{T_{\Delta}}$. In this situation we have $\mathrm{DH}_{\mathfrak{c}_{-}}=-\mathbf{D}_{Z}$ where the polynomial $\mathbf{D}_{Z}$ is defined by (1.23).

\subsection{Quantum version of Duistermaat-Heckman measures}

We suppose here that the Hamiltonian $T$-manifold $(M, \omega, \Phi)$ is prequantized by a $T$-equivariant Hermitian line bundle $L$ over $M$, which is equipped with an Hermitian connection $\nabla$ satisfying the Kostant formula

$$
\mathcal{L}(X)-\nabla_{X_{M}}=i\langle\Phi, X\rangle, \quad X \in \mathfrak{t} .
$$

The former equation implies that the first Chern class of $L$ is equal to $\frac{\Omega}{2 \pi}$. In this section we suppose that $M$ is compact and we still assume that the generic stabiliser $\Gamma_{M}$ of $T$ on $M$ is finite. The quantization of $(M, \Omega)$ is defined by the Riemann-Roch character $R R(M, L) \in R(T)$ which is compute with a $T$ equivariant almost complex stucture on $M$ compatible with $\Omega$ [32]. For $k \geq 1$, we consider the tensor product $L^{\otimes k}$. Its Riemann-Roch character $R R\left(M, L^{\otimes k}\right)$ decomposes as

$$
R R\left(M, L^{\otimes k}\right)=\sum_{\mu \in \Lambda^{*}} \mathrm{~m}(\mu, k) \mathbb{C}_{\mu}
$$


Let us recall the well-known properties of the map $\mathrm{m}: \Lambda^{*} \times \mathbb{Z}^{>0} \rightarrow \mathbb{Z}$. When $\frac{\mu}{k}$ is a regular value of $\Phi$, the "Quantization commutes with Reduction Theorem" [28, 29] tell us that

$$
\mathrm{m}(\mu, k)=R R\left(\mathcal{M}_{\frac{\mu}{k}}, \mathcal{L}^{\mu, k}\right)
$$

where $\mathcal{L}^{\mu, k}=\left(\left.L^{\otimes k}\right|_{\Phi^{-1}\left(\frac{\mu}{k}\right)} \otimes \mathbb{C}_{-\mu}\right) / T$ is an orbifold line bundle over the symplectic orbifold $\mathcal{M}_{\frac{\mu}{k}}=\Phi^{-1}\left(\frac{\mu}{k}\right) / T$. In particular if $\frac{\mu}{k}$ does not belong to $\Phi(M)$ we have $\mathrm{m}(\mu, k)=0$. When $\frac{\mu}{k} \in \Phi(M)$ is not necessarilly a regular value of $\Phi$, one procceed by shift desingularization. If $\xi \in \Phi(M)$ is a regular value of $\Phi$ close enough to $\frac{\mu}{k}$ then (1.27) becomes

$$
\mathrm{m}(\mu, k)=R R\left(\mathcal{M}_{\xi}, \mathcal{L}_{\xi}^{\mu, k}\right)
$$

where $\mathcal{L}_{\xi}^{\mu, k}=\left(\left.L^{\otimes k}\right|_{\Phi^{-1}(\xi)} \otimes \mathbb{C}_{-\mu}\right) / T$ (for a proof see $\left.[29,32]\right)$.

Definition 1.3.1 A function $f: \Xi \rightarrow \mathbb{Z}$ defined over a lattice $\Xi \simeq \mathbb{Z}^{r}$ is called periodic polynomial if

$$
f(x)=\sum_{i=1}^{p} e^{i \frac{\left\langle\alpha_{j}, x\right\rangle}{N}} P_{j}(x), \quad x \in \Xi,
$$

where $\alpha_{1}, \cdots, \alpha_{p} \in \Xi^{*}, N \geq 1$, and the functions $P_{1}, \cdots, P_{p}$ are polynomials with complex coefficients.

Remark 1.3.2 Let $\mathcal{C}$ a cone with non-empty interior in the real vector space $\Xi \otimes_{\mathbb{Z}} \mathbb{R}$. Any periodic-polynomial function $f: \Xi \rightarrow \mathbb{Z}$ is completely determined by its restriction on $\mathcal{C} \cap \Xi$.

Let $\mathfrak{c} \subset \mathfrak{t}^{*}$ be a connected component of regular values of $\Phi$. In [29] Meinrenken an Sjamaar proved that there exits a periodic polynomial function $\mathrm{m}_{\mathfrak{c}}: \Lambda^{*} \times \mathbb{Z} \rightarrow \mathbb{Z}$ such that $\mathrm{m}_{\mathfrak{c}}(\mu, k)=\mathrm{m}(\mu, k)$ for every $(\mu, k)$ in the cone

$$
\operatorname{Cone}(\mathfrak{c})=\left\{(\xi, s) \in \mathfrak{t}^{*} \times \mathbb{R}^{>0} \mid \xi \in s \cdot \mathfrak{c}\right\}
$$

Consider now two adjacent connected regions $\mathfrak{c}_{ \pm}$of regular values of $\Phi$ separated by an hyperplane $\Delta \subset \mathfrak{t}^{*}$. When $\Delta$ does not contain a facet of the polytope $\Phi(M)$, Meinrenken an Sjamaar proved also that

$$
\mathrm{m}_{\mathfrak{c}_{+}}(\mu, k)=\mathrm{m}_{\mathfrak{c}_{-}}(\mu, k)=\mathrm{m}(\mu, k)
$$

for every $(\mu, k) \in \overline{\operatorname{Cone}\left(\mathfrak{c}_{+}\right)} \cap \overline{\operatorname{Cone}\left(\mathfrak{c}_{-}\right)}=\operatorname{Cone}\left(\overline{\mathfrak{c}_{+}} \cap \overline{\mathfrak{c}_{-}}\right) \subset \operatorname{Cone}(\Delta)$.

The main objective of this section is to prove that (1.30) extends to a "strip" containing Cone $(\Delta)$.

Let $\beta \in \Lambda$ be the primitive orthogonal vector to the hyperplane $\Delta \subset \mathfrak{t}^{*}$ which is pointing out of $\mathfrak{c}_{-}$. Then $\Delta=\left\{\xi \in \mathfrak{t}^{*} \mid \frac{\langle\xi, \beta\rangle}{2 \pi}=r_{\Delta}\right\}$ for some $r_{\Delta} \in \mathbb{Z}$, Cone $(\Delta)=\left\{(\xi, s) \in \mathfrak{t}^{*} \times \mathbb{R}^{\geq 0} \mid \frac{\langle\xi, \beta\rangle}{2 \pi}-s r_{\Delta}=0\right\}$ and $\mathfrak{c}_{-} \subset\left\{\xi \in \mathfrak{t}^{*} \mid \frac{\langle\xi, \beta\rangle}{2 \pi}<r_{\Delta}\right\}$. 
Let $T_{\Delta}$ be the subtorus of $T$ generated by $\beta$. Let $N_{\Delta}$ be the normal vector bundle of $M^{T \Delta}$ in $M$. The almost complex structure on $M$ induces a complex structure $J$ on the fibers of $N_{\Delta}$. We have a decomposition $N_{\Delta}=\sum_{s} N_{\Delta}^{s}$ where $N_{\Delta}^{s}=\left\{v \in N_{\Delta} \mid \mathcal{L}_{\beta} v=s J v\right\}$. We write $N_{\Delta}=N_{\Delta}^{+, \beta} \oplus N_{\Delta}^{-, \beta}$ where

$$
N_{\Delta}^{ \pm, \beta}=\sum_{ \pm s>0} N_{\Delta}^{s}
$$

Definition 1.3.3 For every connected component $Z \subset M^{T \Delta}$ we define $s_{Z}^{ \pm} \in$ $\mathbb{N}$ respectively as the absolute value of the trace of $\frac{1}{2 \pi} \mathcal{L}_{\beta}$ on $\left.N_{\Delta}^{ \pm, \beta}\right|_{Z}$.

Note that $s_{Z}^{+}+s_{Z}^{-}$is larger than half of the codimension of $Z$ in $M$. We prove in Section 1.3.5 the following

Theorem 1.3.4 We have $\mathrm{m}_{\mathfrak{c}_{+}}(\mu, k)=\mathrm{m}_{\mathfrak{c}_{-}}(\mu, k)$ for all $(\mu, k) \in \Lambda^{*} \times \mathbb{Z}$ such that

$$
-s^{-}<\frac{\langle\mu, \beta\rangle}{2 \pi}-k r_{\Delta}<s^{+} .
$$

The number $s^{-}, s^{+} \in \mathbb{N}$ are defined as follows. We take $s^{ \pm}=\inf _{Z} s_{Z}^{ \pm}$where the minimum is taken over the connected components $Z$ of $M^{T_{\Delta}}$ for which $\overline{\mathfrak{c}_{+}} \cap \overline{\mathfrak{c}_{-}} \subset \Phi(Z)$.

Similar results were obtained by Billey-Guillemin-Rassart [10] in the case where $M$ is a coadjoint orbit of $\mathrm{SU}(n)$, and by Szenes-Vergne [36] in the case where $M$ is a complex vector space. See Sections 1.4.4 and 1.5 where we study these two particular cases in details. In Proposition 1.3.25, we give also a criterium which says when the inequalities in (1.32) are optimal. This criterium is fullfilled when there is only one component $Z$ of $M^{T_{\Delta}}$ such that $\overline{\mathfrak{c}_{+}} \cap \overline{\mathfrak{c}_{-}} \subset \Phi(Z)$. Then (1.32) is optimal and $s^{+}+s^{-}$is larger than half of the codimension of $Z$ in $M$.

The following easy Lemma (see Lemma 7.3. of [32]) gives some basic informations about the integer $s_{Z}^{ \pm}$.

Lemma 1.3.5 Let $(M, \Omega, \Phi)$ be a compact Hamiltonian T-manifold equipped with a $T$-invariant almost complex structure compatible with $\Omega$. Consider a non-zero vector $\gamma \in \mathfrak{t}$ and let $Z$ be a connected component of the fixed point set $M^{\gamma}$. Let $N$ be the normal vector of $Z$ in $M$ and let $N^{-, \gamma}$ be the negative polarized normal bundle (see (1.31)). Then $N^{-, \gamma}=0$ if and only if the function $\langle\Phi, \gamma\rangle: M \rightarrow \mathbb{R}$ takes its maximal value on $Z$.

This Lemma insures that $s^{ \pm} \geq 1$ in Theorem 1.3.4 when $\Delta \cap \Phi(M)$ is not a facet of the polytope $\Phi(M)$.

Consider the situation where $\Delta \cap \Phi(M)$ is a facet of the polytope $\Phi(M)$ so that $\mathfrak{c}_{+} \cap \Phi(M)=\emptyset$ : hence $\mathrm{m}_{\mathfrak{c}_{+}}=0$. If we apply Lemma 1.3 .5 with $\gamma=\beta$, one gets $N^{-, \beta}=0$ and so $s^{-}=0$. In this situation we get 
Corollary 1.3.6 Let $\mathfrak{c}_{-}$be a connected component of regular values of $\Phi$ bording a facet $\Phi(M) \cap \Delta$ of the polytope $\Phi(M)$. Let $\beta \in \Lambda$ be the primitive orthogonal vector to the hyperplane $\Delta \subset \mathfrak{t}^{*}$ which is pointing out of $\mathfrak{c}_{-}$. Here $Z=\Phi^{-1}(\Delta)$ is a connected component of the fixed point set $M^{T_{\Delta}}$. We have $\mathrm{m}_{\mathfrak{c}_{-}}(\mu, k)=0$ for all $(\mu, k) \in \Lambda^{*} \times \mathbb{Z}$ such that

$$
0<\frac{\langle\mu, \beta\rangle}{2 \pi}-k r_{\Delta}<s_{Z}^{+}
$$

Here $s_{Z}^{+} \in \mathbb{N}$ is larger than half of the codimension of $Z$ in $M$, and the inequalities (1.33) are optimal.

The rest of this section is dedicated to the proof of Theorem 1.3.4. We start by reviewing some of the results of [32].

\subsubsection{Elliptic and transversally elliptic symbols}

We work in the setting of a compact manifold $M$ equipped with a smooth action of a torus $T$.

Let $p: \mathbf{T} M \rightarrow M$ be the projection, and let $(\cdot, \cdot)_{M}$ be a $T$-invariant Riemannian metric. If $E^{0}, E^{1}$ are $T$-equivariant vector bundles over $M$, a $T$ equivariant morphism $\sigma \in \Gamma\left(\mathbf{T} M, \operatorname{hom}\left(p^{*} E^{0}, p^{*} E^{1}\right)\right)$ is called a symbol. The subset of all $(m, v) \in \mathbf{T} M$ where $\sigma(m, v): E_{m}^{0} \rightarrow E_{m}^{1}$ is not invertible is called the characteristic set of $\sigma$, and is denoted by $\operatorname{Char}(\sigma)$.

Let $\mathbf{T}_{T} M$ be the following subset of $\mathbf{T} M$ :

$$
\mathbf{T}_{T} M=\left\{(m, v) \in \mathbf{T} M,\left(v, X_{M}(m)\right)_{M}=0 \quad \text { for all } X \in \mathfrak{k}\right\} .
$$

A symbol $\sigma$ is elliptic if $\sigma$ is invertible outside a compact subset of $\mathbf{T} M$ (Char $(\sigma)$ is compact), and is transversally elliptic if the restriction of $\sigma$ to $\mathbf{T}_{T} M$ is invertible outside a compact subset of $\mathbf{T}_{T} M\left(\operatorname{Char}(\sigma) \cap \mathbf{T}_{T} M\right.$ is compact). An elliptic symbol $\sigma$ defines an element in the equivariant $K$-theory of $\mathbf{T} M$ with compact support, which is denoted by $\mathbf{K}_{T}(\mathbf{T} M)$, and the index of $\sigma$ is a virtual finite dimensional representation of $T[3,4,5,6]$.

A transversally elliptic symbol $\sigma$ defines an element of $\mathbf{K}_{T}\left(\mathbf{T}_{T} M\right)$, and the index of $\sigma$ is defined as a trace class virtual representation of $T$ (see [1] for the analytic index and $[8,9]$ for the cohomological one). Remark that any elliptic symbol of $\mathbf{T} M$ is transversally elliptic, hence we have a restriction map $\mathbf{K}_{T}(\mathbf{T} M) \rightarrow \mathbf{K}_{T}\left(\mathbf{T}_{T} M\right)$, and a commutative diagram

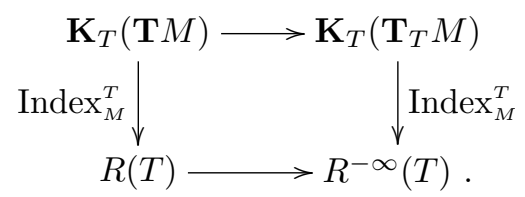

Using the excision property, one can easily show that the index map Index $\mathcal{U}_{\mathcal{U}}^{T}: \mathbf{K}_{T}\left(\mathbf{T}_{T} \mathcal{U}\right) \rightarrow R^{-\infty}(T)$ is still defined when $\mathcal{U}$ is a $T$-invariant relatively compact open subset of a $T$-manifold (see [32] [section 3.1]). 


\subsubsection{Localization of the Riemann-Roch character}

We suppose now that the compact $T$-manifold $M$ is equipped with a $T$ invariant almost complex structure $J$. Let us recall the definitions of the Thom symbol $\operatorname{Thom}(M, J)$ and of the Riemann-Roch character [32].

Consider a $T$-invariant Riemannian metric $q$ on $M$ such that $J$ is orthogonal relatively to $q$, and let $h$ be the Hermitian structure on $\mathbf{T} M$ defined by $: h(v, w)=q(v, w)-\imath q(J v, w)$ for $v, w \in \mathbf{T} M$. The symbol

$$
\operatorname{Thom}(M, J) \in \Gamma\left(M, \operatorname{hom}\left(p^{*}\left(\wedge_{\mathbb{C}}^{\text {even }} \mathbf{T} M\right), p^{*}\left(\wedge_{\mathbb{C}}^{\text {odd }} \mathbf{T} M\right)\right)\right)
$$

at $(m, v) \in \mathbf{T} M$ is equal to the Clifford map

$$
\mathrm{Cl}_{m}(v): \wedge_{\mathbb{C}}^{\text {even }} \mathbf{T}_{m} M \longrightarrow \wedge_{\mathbb{C}}^{\text {odd }} \mathbf{T}_{m} M
$$

where $\mathrm{Cl}_{m}(v) \cdot w=v \wedge w-c_{h}(v) \cdot w$ for $w \in \wedge_{\mathbb{C}}^{\bullet} \mathbf{T}_{x} M$. Here $c_{h}(v): \wedge_{\mathbb{C}}^{\bullet} \mathbf{T}_{m} M \rightarrow$ $\wedge^{\bullet-1} \mathbf{T}_{m} M$ denotes the contraction map relative to $h$. Since the map $\mathrm{Cl}_{m}(v)$ is invertible for all $v \neq 0$, the symbol $\operatorname{Thom}(M, J)$ is elliptic.

The Riemann-Roch character $R R(M,-): \mathbf{K}_{T}(M) \rightarrow R(T)$ is defined by the following relation

$$
R R(M, E)=\operatorname{Index}_{M}^{T}\left(\operatorname{Thom}(M, J) \otimes p^{*} E\right) .
$$

The important point is that for any $T$-vector bundle $E$, Thom $(M, J) \otimes p^{*} E$ corresponds to the principal symbol of the twisted $\operatorname{Spin}^{c}$ Dirac operator $\mathcal{D}_{E}$ [16], hence $R R(M, E) \in R(T)$ is also defined as the (analytical) index of the elliptic operator $\mathcal{D}_{E}$.

Consider now the case of a compact Hamiltonian $T$-manifold $(M, \omega, \Phi)$. Here $J$ is a $T$-invariant almost comlex structure compatible with $\Omega:(v, w) \mapsto$ $\Omega(v, J w)$ defines a Riemannian metric on $M$. Like in Section 1.2.2, we make the choice of a scalar product $(\cdot, \cdot)$ on $\mathfrak{t}^{*}$ (which induces an identification $\left.\mathfrak{t}^{*} \simeq \mathfrak{t}\right)$ and we consider for any $\xi \in \mathfrak{t}^{*}$ the function $\frac{-1}{2}\|\Phi-\xi\|^{2}: M \rightarrow \mathbb{R}$ and its Hamiltonian vector field $\mathcal{H}-\xi_{M}$.

Definition 1.3.7 For any $\xi \in \mathfrak{t}^{*}$ and any $T$-invariant open subset $\mathcal{U} \subset M$ we define the symbol $\operatorname{Thom}_{\xi}(\mathcal{U})$ by the relation

$\operatorname{Thom}_{\xi}(\mathcal{U})(m, v):=\operatorname{Thom}(M, J)\left(m, v-\left(\mathcal{H}-\xi_{M}\right)(m)\right) \quad(m, v) \in \mathbf{T} \mathcal{U}$

The characteristic set of $\operatorname{Thom}_{\xi}(\mathcal{U})$ corresponds to $\{(m, v) \in \mathbf{T} \mathcal{U}, v=$ $\left.\left(\mathcal{H}-\xi_{M}\right)(m)\right\}$, the graph of the vector field $\mathcal{H}-\xi_{M}$ over $\mathcal{U}$. Since $\mathcal{H}-\xi_{M}$ belongs to the set of tangent vectors to the $T$-orbits, we have

$$
\begin{aligned}
\operatorname{Char}\left(\operatorname{Thom}_{\xi}(\mathcal{U})\right) \cap \mathbf{T}_{T} \mathcal{U} & =\left\{(m, 0) \in \mathbf{T} \mathcal{U} \mid\left(\mathcal{H}-\xi_{M}\right)(m)=0\right\} \\
& \cong\left\{m \in \mathcal{U}, d\|\Phi-\xi\|_{m}^{2}=0\right\} .
\end{aligned}
$$

Therefore the $\operatorname{symbol} \operatorname{Thom}_{\xi}(\mathcal{U})$ is transversally elliptic if and only if

$$
\operatorname{Cr}\left(\|\Phi-\xi\|^{2}\right) \cup \partial \mathcal{U}=\emptyset .
$$


Definition 1.3.8 When (1.37) holds we say that the couple $(\mathcal{U}, \xi)$ is good.

Definition 1.3.9 Let $(\mathcal{U}, \xi)$ be a good couple. For any $T$-vector bundle $E \rightarrow$ $M$, the tensor product $\operatorname{Thom}_{\xi}(\mathcal{U}) \otimes p^{*} E$ belongs to $\mathbf{K}_{T}\left(\mathbf{T}_{T} \mathcal{U}\right)$ and we denote by

$$
R R_{\mathcal{U}}^{\xi}(M, E) \in R^{-\infty}(T)
$$

its index.

Proposition 1.3.10 Let $(\mathcal{U}, \xi)$ be a good couple.

- If $\mathcal{U}$ possess two $T$-invariant open subsets $\mathcal{U}^{1}, \mathcal{U}^{2}$ such that $\overline{\mathcal{U}^{1}} \cap \overline{\mathcal{U}^{2}} \cap$ $\operatorname{Cr}\left(\|\Phi-\xi\|^{2}\right)=\emptyset$ and $\left(\mathcal{U}^{1} \cup \mathcal{U}^{2}\right) \cap \operatorname{Cr}\left(\|\Phi-\xi\|^{2}\right)=\mathcal{U} \cap \operatorname{Cr}\left(\|\Phi-\xi\|^{2}\right)$, then the couples $\left(\mathcal{U}^{1}, \xi\right)$ and $\left(\mathcal{U}^{2}, \xi\right)$ are good and

$$
R R_{\mathcal{U}}^{\xi}(M,-)=R R_{\mathcal{U}^{1}}^{\xi}(M,-)+R R_{\mathcal{U}^{2}}^{\xi}(M,-) .
$$

In particular $R R_{\mathcal{U}}^{\xi}(M,-)=R R_{\mathcal{U}^{1}}^{\xi}(M,-)$ if $\mathcal{U}^{1}$ is an open subset of $\mathcal{U}$ such that $\mathcal{U}^{1} \cap \operatorname{Cr}\left(\|\Phi-\xi\|^{2}\right)=\mathcal{U} \cap \operatorname{Cr}\left(\|\Phi-\xi\|^{2}\right)$.

- If $\xi^{\prime} \in \mathfrak{t}^{*}$ is close enough to $\xi$, then $\left(\mathcal{U}, \xi^{\prime}\right)$ is good and

$$
R R_{\mathcal{U}}^{\xi}(M,-)=R R_{\mathcal{U}}^{\xi^{\prime}}(M,-) .
$$

Proof. The first point is a direct consequence of the excision property (see Proposition 4.1. in [32]).

Let us prove the second point. Consider now the scalar product

$$
\phi(s):=\left(\mathcal{H}-\xi_{M}^{s}, \mathcal{H}-\xi_{M}\right)_{M}
$$

where $\xi^{s}=s \xi^{\prime}+(1-s) \xi, s \in[0,1]: \phi(s)$ is a smooth function on $M$. We have $\phi(s)=\left\|\mathcal{H}-\xi_{M}\right\|^{2}+s\left(\left(\xi-\xi^{\prime}\right)_{M}, \mathcal{H}-\xi_{M}\right)$ and then the following inequality holds on $M$

$$
\phi(s) \geq\left\|\mathcal{H}-\xi_{M}\right\|^{2}\left(\left\|\mathcal{H}-\xi_{M}\right\|-s\left\|\xi_{M}-\xi_{M}^{\prime}\right\|\right) .
$$

Since $\partial \mathcal{U}$ is compact we have the following inequalities on it: $\left\|\mathcal{H}-\xi_{M}\right\| \geq c_{1}>$ 0 and $\left\|X_{M}\right\| \leq c_{2}\|X\|$ for any $a \in \mathfrak{t}$. So (1.38) implies the following inequality on $\partial \mathcal{U}$ :

$$
\phi(s) \geq c_{1}\left(c_{1}-s\left\|\xi-\xi^{\prime}\right\|\right) \quad \text { for } \quad s \in[0,1] .
$$

So if $\xi^{\prime}$ is close enough to $\xi$, we have $\left\|\mathcal{H}-\xi_{M}^{s}\right\| \geq c_{3}>0$ on $\partial \mathcal{U}$ for any $s \in[0,1]$. We have first prove that the couple $\left(\mathcal{U}, \xi^{s}\right)$ is good for any $s \in[0,1]$. We see then that the familly of transversally elliptic symbols $\operatorname{Thom}_{\xi^{s}}(\mathcal{U}), s \in[0,1]$ defines an homotopy between $\operatorname{Thom}_{\xi}(\mathcal{U})$ and $\operatorname{Thom}_{\xi^{\prime}}(\mathcal{U})$. Hence $\operatorname{Thom}_{\xi}(\mathcal{U})=$ $\operatorname{Thom}_{\xi^{\prime}}(\mathcal{U})$ in $\mathbf{K}_{T}\left(\mathbf{T}_{T} \mathcal{U}\right)$.

The first point of Proposition 1.3.10 shows that $R R_{\mathcal{U}}^{\xi}(M,-)$ depends closely of the intersection $\mathcal{U} \cap \operatorname{Cr}\left(\|\Phi-\xi\|^{2}\right)$. In particular $R R_{\mathcal{U}}^{\xi}(M,-)=0$ when $\mathcal{U} \cap \operatorname{Cr}\left(\|\Phi-\xi\|^{2}\right)=\emptyset$. Recall that 


$$
\operatorname{Cr}\left(\|\Phi-\xi\|^{2}\right)=\bigcup_{\gamma \in \mathcal{B}_{\xi}} M^{\gamma} \cap \Phi^{-1}(\gamma+\xi)
$$

where $\mathcal{B}_{\xi} \subset \mathfrak{t}^{*}$ is a finite set [24].

Definition 1.3.11 For any $\xi \in \mathfrak{t}^{*}$ and $\gamma \in \mathcal{B}_{\xi}$, we denote simply by

$$
R R_{\gamma}^{\xi}(M,-): \mathbf{K}_{T}(M) \rightarrow R^{-\infty}(T)
$$

the map $R R_{\mathcal{U}}^{\xi}(M,-)$, where $\mathcal{U}$ is a T-invariant open neighborhood of $M^{\gamma} \cap$ $\Phi^{-1}(\gamma+\xi)$ such that $\operatorname{Cr}\left(\|\Phi-\xi\|^{2}\right) \cap \overline{\mathcal{U}}=M^{\gamma} \cap \Phi^{-1}(\gamma+\xi)$.

Proposition 1.3.10 insures that the maps $R R_{\gamma}^{\xi}(M,-)$ are well defined, and for any good couple $(\mathcal{U}, \xi)$ we have

$$
R R_{\mathcal{U}}^{\xi}(M,-)=\sum_{\gamma \in \mathcal{B}_{\xi} \cap \Phi(\mathcal{U})} R R_{\gamma}^{\xi}(M,-) .
$$

If one takes $\mathcal{U}=M$, we have $R R_{\mathcal{U}}^{\xi}(M,-)=R R(M,-)=\sum_{\gamma \in \mathcal{B}_{\xi}} R R_{\gamma}^{\xi}(M,-)$ (see [32][Section 4]).

\subsubsection{Periodic polynomial behaviour of the multiplicities}

We suppose here that the Hamiltonian $T$-manifold $(M, \Omega, \Phi)$ is prequantized by a $T$-complex line bundle $L$ satisfying (1.25) for a suitable invariant connection. In this section we will characterize the periodic polynomial behaviour of the multiplicities $\mathrm{m}(\mu, k)$ with the help of the localized Riemann-Roch character $R R_{0}^{\xi}(M,-)$.

Let us introduce some vocabulary. We say that two generalized characters $\chi^{ \pm}=\sum_{\mu \in \Lambda^{*}} a_{\mu}^{ \pm} \mathbb{C}_{\mu}$ coincide on a region $D \subset \mathfrak{t}^{*}$, if $a_{\mu}^{+}=a_{\mu}^{-}$for every $\mu \in$ $D \cap \Lambda^{*}$. A generalized character $\chi=\sum_{\mu} a_{\mu} \mathbb{C}_{\mu}$ is supported on a region $D \subset \mathfrak{t}^{*}$ if $a_{\mu}=0$ for $\mu \notin D$. A weight $\mu \in \Lambda^{*}$ occurs in $\chi=\sum_{\mu} a_{\mu} \mathbb{C}_{\mu}$ if $a_{\mu} \neq 0$.

For $\xi \in \mathfrak{t}^{*}$, we define $r_{\xi}>0$ as the smallest non-zero critical value of the function $\|\Phi-\xi\|$, and we denote by $B\left(\xi, r_{\xi}\right)$ the open ball of center $\xi$ and radius $r_{\xi}$.

Theorem 1.3.12 ([32]) For any $\xi \in \mathfrak{t}^{*}$, the generalized character $R R_{0}^{\xi}\left(M, L^{\otimes k}\right)$ coincides with $R R\left(M, L^{\otimes k}\right)$ on the open ball $k \cdot B\left(\xi, r_{\xi}\right)$.

The arguments of [32] for the proof of this Theorem will be needed another time, so we recall them. Let $\xi \in \mathfrak{t}^{*}$. We start with the decomposition

$$
R R\left(M, L^{\otimes k}\right)=\sum_{\gamma \in \mathcal{B}_{\xi}} R R_{\gamma}^{\xi}\left(M, L^{\otimes k}\right) .
$$

We recall now, for a non-zero $\gamma \in \mathcal{B}_{\xi}$, the localization of the map $R R_{\gamma}^{\xi}$ on the fixed point set $M^{\gamma}[32]$. 
Let $N$ be the normal bundle of $M^{\gamma}$ in $M$. The almost complex structure on $M$ induces an almost complex struture on $M^{\gamma}$ and a complex structure on the bundles $N$ and $N_{\mathbb{C}}:=N \otimes \mathbb{C}$. Following (1.31) we define the $\gamma$-polarized complex vector bundles $N^{+, \gamma}$ and $\left(N_{\mathbb{C}}\right)^{+, \gamma}$.

The manifold $M^{\gamma}$ is a symplectic submanifold of $M$ equipped with an induced Hamiltonian action of $T$ : its moment map is the restriction of $\Phi$ on $M^{\gamma}$. Following Definition 1.3.11, we have on $M^{\gamma}$ a localized Riemann-Roch character $R R_{\gamma}^{\xi}\left(M^{\gamma},-\right)$. On $M^{\gamma}$, the Hamiltonian vector fields of the functions $\|\Phi-\xi\|^{2}$ and $\|\Phi-(\xi+\gamma)\|^{2}$ coincide, hence

$$
R R_{\gamma}^{\xi}\left(M^{\gamma},-\right)=R R_{0}^{\xi+\gamma}\left(M^{\gamma},-\right) .
$$

We prove in [32][Theorem 5.8.] that

$$
R R_{\gamma}^{\xi}(M, E)=\sum_{k \in \mathbb{N}}(-1)^{l} R R_{\gamma}^{\xi}\left(M^{\gamma},\left.E\right|_{M^{\gamma}} \otimes \operatorname{det}\left(N^{+, \gamma}\right) \otimes S^{k}\left(N_{\mathbb{C}}^{+, \gamma}\right)\right)
$$

for every $T$-vector bundle $E$. Here $l$ is the locally constant fonction on $M^{\gamma}$ equal to the complex rank of $N^{+, \gamma}$.

Proposition 1.3.13 ([32], Section 5) Let $\bar{N}$ be the T-vector bundle $N$ with the opposite complex structure on the fibers. The sum $(-1)^{l} \sum_{k \in \mathbb{N}} \operatorname{det}\left(N^{+, \gamma}\right) \otimes$ $S^{k}\left(N_{\mathbb{C}}^{+, \gamma}\right)$ is an inverse of $\wedge_{\mathbb{C}}^{\bullet} \bar{N}$ that we denote $\left[\wedge_{\mathbb{C}}^{\bullet} \bar{N}\right]_{\gamma}^{-1}$.

If we use the notations of Proposition 1.3.13 and (1.42), the localization (1.43) can be rewritten as

$$
R R_{\gamma}^{\xi}(M, E)=R R_{0}^{\xi+\gamma}\left(M^{\gamma},\left.E\right|_{M^{\gamma}} \otimes\left[\wedge_{\mathbb{C}} \bar{N}\right]_{\gamma}^{-1}\right) .
$$

Let $i: T_{\gamma} \hookrightarrow T$ be the inclusion of the subtorus generated by $\gamma$. Let $F$ be a $T$-vector bundle on $M^{\gamma}$.

Lemma 1.3.14 ([32], Lemma 9.4.) A weight $\mu \in \Lambda^{*}$ occurs in $R R_{\gamma}^{\xi}\left(M^{\gamma}, F\right)$ only if $i^{*}(\mu)$ occurs as a weight for the $T_{\gamma}$-action on the fibers of $F \otimes\left[\wedge{ }_{\mathbb{C}} \bar{N}\right]_{\gamma}^{-1}$.

Since the $T_{\gamma}$ weights on the bundles $N_{\mathbb{C}}^{+, \gamma}$ and $N^{+, \gamma}$ are polarized by $\gamma$, the localization (1.43) gives the following

Corollary 1.3.15 For a non-zero $\gamma \in \mathcal{B}_{\xi}$, the generalized character $R R_{\gamma}^{\xi}\left(M, L^{\otimes k}\right)$ is supported on the half space $\left\{a \in \mathfrak{t}^{*} \mid(\gamma, a-k(\xi+\gamma)) \geq 0\right\}$.

Since the condition $(\gamma, a-k(\xi+\gamma)) \geq 0$ implies that $\|a-k \xi\| \geq k\|\gamma\| \geq$ $k r_{\xi}$, the last proposition shows that every weights of the open ball $k \cdot B\left(\xi, r_{\xi}\right)$ does not occurs in $R R_{\gamma}^{\xi}\left(M, L^{\otimes k}\right)$. This last remark together with (1.41) prove Theorem 1.3.12.

For the localized Riemann-Roch character $R R_{0}^{\xi}(M,-)$ we have the following Lemma which is very similar to Lemma 1.2.3. 
Lemma 1.3.16 Let $\mathfrak{c} \subset \mathfrak{t}^{*}$ be a connected component of regular values of $\Phi$. For every $\xi, \xi^{\prime} \in \mathfrak{c}$, we have $R R_{0}^{\xi}(M,-)=R R_{0}^{\xi^{\prime}}(M,-)$.

Proof. We have to show that the map $\xi \mapsto R R_{0}^{\xi}(M,-)$ is locally constant on $\mathfrak{c}$. Let $\xi \in \mathfrak{c}$ and take an open neigborhood $\mathcal{U}$ of $\Phi^{-1}(\xi)$ small enough such that the stabilizer $T_{m}=\{t \in T \mid t \cdot m=m\}$ is finite for every $m \in \overline{\mathcal{U}}$. We see then that $\overline{\mathcal{U}} \cap \operatorname{Cr}\left(\left\|\Phi-\xi^{\prime}\right\|^{2}\right)=\Phi^{-1}\left(\xi^{\prime}\right)$ and $\partial \mathcal{U} \cap \operatorname{Cr}\left(\left\|\Phi-\xi^{\prime}\right\|^{2}\right)=\emptyset$ if $\xi^{\prime}$ is close enough to $\xi$ : hence $R R_{0}^{\xi^{\prime}}(M,-)=R R_{\mathcal{U}}^{\xi^{\prime}}(M,-)$ for $\xi^{\prime}$ close enough to $\xi$. The second point of Proposition 1.3.10 finishes the proof.

When $\xi$ is a regular value of $\Phi$, the localized Riemann-Roch character $R R_{0}^{\xi}(M,-)$ as been computed in [32] as follows. Let $R R\left(\mathcal{M}_{\xi},-\right)$ be the Riemann-Roch map defined on the orbifold $\mathcal{M}_{\xi}=\Phi^{-1}(\xi) / T$ by means of an almost complex structure compatible with the induced symplectic structure. For every $T$-vector bundle $E \rightarrow M$ we define the following familly of orbifold vector bundles over $\mathcal{M}_{\xi}$ :

$$
\mathcal{E}_{\xi}^{\mu}:=\left(\left.E\right|_{\Phi^{-1}(\xi)} \otimes \mathbb{C}_{-\mu}\right) / T, \quad \mu \in \Lambda^{*} .
$$

For every $T$-vector bundle $E$ on $M$, we proved in [32][Section 6.2.] the following equality in $R^{-\infty}(T)$

$$
R R_{0}^{\xi}(M, E)=\sum_{\mu \in \Lambda^{*}} R R\left(\mathcal{M}_{\xi}, \mathcal{E}_{\xi}^{\mu}\right) \mathbb{C}_{\mu} .
$$

This decomposition was first obtained by Vergne [37] when $T$ is the circle group and when $M$ is Spin. The number $R R\left(\mathcal{M}_{\xi}, \mathcal{E}_{\xi}^{\mu}\right) \in \mathbb{Z}$ is then equal to the $T$-invariant part of the index $R R_{0}^{\xi}(M, E) \otimes \mathbb{C}_{-\mu}$.

Remark 1.3.17 Let $t \rightarrow t^{\lambda}$ be a character of T. Suppose that a subgroup $H \subset T$ acts trivially on $M$ and with the character $t \in H \rightarrow t^{\lambda}$ on the the fibers of the T-vector bundle $E$. Then $H$ acts with the character $t \in H \rightarrow t^{\lambda-\mu}$ on $R R_{0}^{\xi}(M, E) \otimes \mathbb{C}_{-\mu}$, and then $R R\left(\mathcal{M}_{\xi}, \mathcal{E}_{\xi}^{\mu}\right) \neq 0$ only if $t^{\lambda-\mu}=1$ for every $t \in H$. So the sum in (1.46) can be restricted to $\lambda+\Lambda_{H}^{*}$, where $\Lambda_{H}^{*}$ is the sub-lattice of $\Lambda^{*}$ formed by the element $\alpha \in \Lambda^{*}$ satisfying $t^{\alpha}=1, \forall t \in H$.

This remark applies also on the usual character $R R(M, E)=\sum_{\mu \in \Lambda^{*}} m_{\mu} \mathbb{C}_{\mu}$. The multiplicity $m_{\mu} \in \mathbb{Z}$ is equal to the (virtual) dimension of the T-invariant part of $R R(M, E) \otimes \mathbb{C}_{-\mu}$. With the same hypothesis than above we see that $m_{\mu} \neq 0$ only if $\mu \in \lambda+\Lambda_{H}^{*}$.

Let $\Gamma_{M}$ be the generic stabilizer for the action of $T$ on $M$. Consider a weight $\alpha_{o}$ such that $\Gamma_{M}$ acts on the fibers of $L$ with the character $t \mapsto t^{\alpha_{o}}$. We define the sublattice $\Xi(M, L) \subset \Lambda^{*} \times \mathbb{Z}$ by

$$
\Xi(M, L):=\left\{(\mu, k) \in \Lambda^{*} \times \mathbb{Z} \mid k \alpha_{o}-\mu \in \Lambda_{\Gamma_{M}}^{*}\right\} .
$$

We know then that $\mathrm{m}(\mu, k)=0$ if $(\mu, k) \notin \Xi(M, L)$. 
Proposition 1.3.18 Let $\mathfrak{c}$ be a connected component of regular values of $\Phi$ and let Cone $(\mathfrak{c})$ be the corresponding cone in $\mathfrak{t}^{*} \times \mathbb{R}^{>0}$ (see (1.29)). Let $\xi \in \mathfrak{c}$. For any $(\mu, k) \in \operatorname{Cone}(\mathfrak{c}) \cap \Xi(M, L)$ we have

$$
\mathrm{m}(\mu, k)=R R\left(\mathcal{M}_{\xi}, \mathcal{L}_{\xi}^{\mu, k}\right)
$$

where

$$
\mathcal{L}_{\xi}^{\mu, k}=\left(\left.L^{\otimes k}\right|_{\Phi^{-1}(\xi)} \otimes \mathbb{C}_{-\mu}\right) / T .
$$

Proof. Let $(\mu, k) \in \operatorname{Cone}(\mathfrak{c})$ and let $\xi^{\prime}=\frac{\mu}{k} \in \mathfrak{c}$. We known from Theorem 1.3.12 that the generalized character $R R_{0}^{\xi^{\prime}}\left(M, L^{\otimes k}\right)$ coincides with $R R\left(M, L^{\otimes k}\right)$ on the open ball $k \cdot B\left(\xi^{\prime}, r_{\xi^{\prime}}\right)=B\left(\mu, k r_{\xi^{\prime}}\right)$. So $\mathrm{m}(\mu, k)$ is equal to the $\mu$-multiplicity in $R R_{0}^{\xi^{\prime}}\left(M, L^{\otimes k}\right)$. Take now any $\xi \in \mathfrak{c}$. We know after Lemma 1.3.16 that $R R_{0}^{\xi}(M,-)=R R_{0}^{\xi^{\prime}}(M,-)$ and (1.46) shows that the $\mu$-multiplicity in $R R_{0}^{\xi}\left(M, L^{\otimes k}\right)$ is equal to $R R\left(\mathcal{M}_{\xi}, \mathcal{L}_{\xi}^{\mu, k}\right)$.

Definition 1.3.19 Take $\xi \in \mathfrak{c}$. The map $\mathrm{m}_{\mathfrak{c}}: \Lambda^{*} \times \mathbb{Z} \rightarrow \mathbb{Z}$ is defined by the equation

$$
\mathrm{m}_{\mathfrak{c}}(\mu, k)=R R\left(\mathcal{M}_{\xi}, \mathcal{L}_{\xi}^{\mu, k}\right),
$$

where $\mathcal{L}_{\xi}^{\mu, k}$ is the orbifold line bundle defined by (1.49).

In other words, the map $\mathrm{m}_{\mathfrak{c}}$ is defined by the following equality in $R^{-\infty}(T)$

$$
\sum_{\mu \in \Lambda^{*}} \mathrm{~m}_{\mathfrak{c}}(\mu, k) \mathbb{C}_{\mu}=R R_{0}^{\xi}\left(M, L^{\otimes k}\right) .
$$

for all $k \in \mathbb{Z}$. After remark 1.3.17, we know that $\mathrm{m}_{\mathfrak{c}}$ is supported on the sub-lattice $\Xi(M, L)$ defined in (1.47).

We will now exploit the Riemann-Roch for orbifold due to Kawasaki [23] to show that the map $\mathrm{m}_{\mathfrak{c}}$ is a periodic polynomial.

\subsubsection{Riemann-Roch-Kawasaki theorem}

First we recall how is defined the Riemann-Roch character $R R\left(\mathcal{M}_{\xi}, \mathcal{E}_{\xi}\right)$ when $\xi$ is a regular value of $\Phi$, and $\mathcal{E}_{\xi}=E_{\mid \Phi^{-1}(\xi)} / T$ is the reduction of a complex $T$-vector bundle $E$ over $M$. The number $R R\left(\mathcal{M}_{\xi}, \mathcal{E}_{\xi}\right) \in \mathbb{Z}$ is defined has the $T$-invariant part of the index of a transversally elliptic operator $D_{E}$ on $\Phi^{-1}(\xi)$. Since the index of $D_{E}$ depend only of the class of its symbol $\sigma\left(D_{E}\right)$ in $K_{T}\left(\mathbf{T}_{T} \Phi^{-1}(\xi)\right)$, it is enough to define the transversally elliptic symbol $\sigma\left(D_{E}\right)$. Since the action of $T$ on $\Phi^{-1}(\xi)$ is locally free, $V:=\mathbf{T}_{T} \Phi^{-1}(\xi)$ is a vector bundle. It carries a canonical symplectic structure on the fibers and we choose any compatible complex structure making $V$ into a Hermitian vector bundle. At $(m, v) \in \mathbf{T} \Phi^{-1}(\xi)$, the map $\sigma\left(D_{E}\right)(m, v)$ is the Clifford action

$$
\mathrm{Cl}_{m}\left(v_{1}\right) \otimes I d_{E_{m}}:\left(\wedge_{\mathbb{C}}^{\text {even }} V_{m}\right) \otimes E_{m} \longrightarrow\left(\wedge_{\mathbb{C}}^{\text {odd }} V_{m}\right) \otimes E_{m} .
$$


where $v_{1} \in V_{m}$ is the $V$-component of the vector $v \in \mathbf{T}_{m} \Phi^{-1}(\xi)$. We explain now the formula of Kawasaki for $R R\left(\mathcal{M}_{\xi}, \mathcal{E}_{\xi}\right)$ when $\xi \in \Phi(M)$ is a regular value of $\Phi[23]$.

Let $\mathbb{F}$ be the collection of the finite subgroup of $T$ which are stabilizer of points in $M$. Consider the orbit type stratification of $\Phi^{-1}(\xi)$ and denote by $\mathbb{S}_{\xi}$ the set of its orbit type strata. Each statum $S$ is a connected component of the smooth submanifold

$$
\Phi^{-1}(\xi)_{H_{S}}:=\left\{m \in \Phi^{-1}(\xi) \mid \operatorname{Stab}_{T}(m)=H_{S}\right\} .
$$

for a unique $H_{S} \in \mathbb{F}$. The orbifold $\mathcal{M}_{\xi}$ decomposes as a disjoint union $\cup_{S \in \mathbb{S}_{\xi}} S / T$ of smooth components, and each quotient $\bar{S} / T$ is a suborbifold of $\mathcal{M}_{\xi}$. The generic stabilizer $\Gamma_{M}$ of $T$ on $M$ is also the generic stabilizer of $T$ on the fiber ${ }^{3} \Phi^{-1}(\xi)$, and is associated to an open and dense stratum $S_{\max }$.

Suppose that $E \rightarrow M$ is an Hermitian $T$-vector bundle. On each suborbifold $\bar{S} / T$, we get the orbifold complex vector bundle

$$
\mathcal{E}_{S}:=E_{\mid \bar{S}} / T
$$

We define twisted characteristic classes $\mathrm{Ch}^{-}\left(\mathcal{E}_{S}\right)$ and $D^{-}\left(\mathcal{E}_{S}\right)$ by

$$
\operatorname{Ch}^{\gamma}\left(\mathcal{E}_{S}\right):=\operatorname{Tr}\left(\gamma^{\mathcal{E}_{S}} \cdot e^{\frac{i}{2 \pi} R\left(\mathcal{E}_{S}\right)}\right), \quad \gamma \in H_{S}
$$

and

$$
D^{\gamma}\left(\mathcal{E}_{S}\right):=\operatorname{det}\left(1-\left(\gamma^{\mathcal{E}_{S}}\right)^{-1} \cdot e^{-\frac{i}{2 \pi} R\left(\mathcal{E}_{S}\right)}\right), \quad \gamma \in H_{S} .
$$

Here $R\left(\mathcal{E}_{S}\right) \in \mathcal{A}^{2}\left(\bar{S} / T, \operatorname{End}\left(\mathcal{E}_{S}\right)\right)$ is the curvature of an horizontal Hermitian connection on $E_{\mid \bar{S}}$, and $\gamma \mapsto \gamma^{\mathcal{E}_{S}}$ is the linear action of $H_{S}$ on the fibers of $E_{\mid \bar{S}}$.

Let $N_{S}$ be the normal bundle of $\bar{S}$ in $\Phi^{-1}(\xi)$. The symplectic struture on $M$ induces a symplectic form $\Omega_{S}$ on each suborbifold $\bar{S} / T$, and a symplectic structure on the fibers of the bundle $N_{S}$. Choose a compatible almost complex structure on $\bar{S} / T$, and a compatible complex structure on the fibers of $N_{S}$ making the tangent bundle of $\bar{S} / T$ and $\mathcal{N}_{S}:=N_{S} / T$ into Hermitian vector bundle. Consider a Hermitian connexion on $\mathbf{T}(\bar{S} / T)$, with curvature $R(\bar{S} / T)$, and let

$$
\operatorname{Todd}(\bar{S} / T)=\operatorname{det}\left(\frac{(i / 2 \pi) R(\bar{S} / T)}{1-e^{-(i / 2 \pi) R(\bar{S} / T)}}\right)
$$

be the corresponding Todd forms. Like in (1.54), we associate to the complex orbifold vector bundle $\mathcal{N}_{S}$, the twisted form $D^{-}\left(\mathcal{N}_{S}\right)$ which is a map form $H_{S}$ to $\mathcal{A}^{\text {even }}(\bar{S} / T)$. The 0 -degree part of $D^{\gamma}\left(\mathcal{N}_{S}\right)$ is equal to $\operatorname{det}\left(1-\left(\gamma^{\mathcal{N}_{S}}\right)^{-1}\right)$, hence $D^{\gamma}\left(\mathcal{N}_{S}\right)$ is invertible in $\mathcal{A}^{\text {even }}(\bar{S} / T)$ when $\gamma$ belongs to

\footnotetext{
${ }^{3}$ Since a neighborhood of $\Phi^{-1}(\xi)$ in $M$ is $T$-equivariantly diffeomorphic to $\Phi^{-1}(\xi) \times$ $t^{*}$.
} 


$$
H_{S}^{o}=\left\{\gamma \in H_{S} \mid \operatorname{det}\left(1-\left(\gamma^{\mathcal{N}_{S}}\right)^{-1}\right) \neq 0\right\} .
$$

Note that $H_{S}^{o}$ corresponds to the set of $\gamma \in H_{S}$ for which $\bar{S}$ is a connected component of $\left(\Phi^{-1}(\xi)\right)^{\gamma}$.

Theorem 1.3.20 (Kawasaki) The number $R R\left(\mathcal{M}_{\xi}, \mathcal{E}_{\xi}\right) \in \mathbb{Z}$ is given by the formula

$$
R R\left(\mathcal{M}_{\xi}, \mathcal{E}_{\xi}\right)=\sum_{S \in \mathbb{S}_{\xi}} \frac{1}{\left|H_{S}\right|} \sum_{\gamma \in H_{S}^{o}} \int_{\bar{S} / T} \frac{\operatorname{Todd}(\bar{S} / T) \mathrm{Ch}^{\gamma}\left(\mathcal{E}_{S}\right)}{D^{\gamma}\left(\mathcal{N}_{S}\right)} .
$$

We exploit now Theorem 1.3.20 to show that the map $\mathrm{m}_{\mathfrak{c}}: \Lambda^{*} \times \mathbb{Z} \rightarrow$ $\mathbb{Z}$ which is defined by (1.50) is periodic polynomial. We need the classical computation of the first Chern class of the line bundle

$$
\mathcal{L}_{S}^{\mu, k}=\left(L^{\otimes k} \otimes \mathbb{C}_{-\mu}\right)_{\mid \bar{S}} / T .
$$

The curvature form $\omega_{\xi} \in H^{2}\left(\mathcal{M}_{\xi}\right) \otimes \mathfrak{t}$ of the principal $T$-bundle $\Phi^{-1}(\xi) \rightarrow \mathcal{M}_{\xi}$ restricts to a curvature form $\omega_{S} \in H^{2}(\bar{S} / T) \otimes \mathfrak{t}$ on each strata.

Lemma 1.3.21 The first Chern class of the line bundle $\mathcal{L}_{S}^{\mu, k}$ is given by

$$
c_{1}\left(\mathcal{L}_{S}^{\mu, k}\right)=\frac{1}{2 \pi}\left(k \Omega_{S}-\left\langle k \xi-\mu, \omega_{S}\right\rangle\right) .
$$

For a strata $S$, we consider $\alpha_{S} \in \Lambda^{*}$ such that $\gamma \in H_{S} \mapsto \gamma^{\alpha_{S}}$ corresponds to the action of $H_{S}$ on the fibers of $L_{\mid \bar{S}}$. Finally we have the decomposition

$$
\mathrm{m}_{\mathfrak{c}}(\mu, k)=\sum_{S \in \mathbb{S}_{\xi}} P_{S}(\mu, k),
$$

where

$$
P_{S}(\mu, k)=\frac{1}{\left|H_{S}\right|} \sum_{\gamma \in H_{S}^{o}} \gamma^{k \alpha_{S}-\mu} \int_{\bar{S} / T} \frac{\operatorname{Todd}(\bar{S} / T)}{D^{\gamma}\left(\mathcal{N}_{S}\right)} e^{\frac{1}{2 \pi}\left(k \Omega_{S}-\left\langle k \xi-\mu, \omega_{S}\right\rangle\right)} .
$$

When $S$ is the principal open dense stratum $S_{\max }$ the map $P_{S}$ is

$$
P_{\max }(\mu, k)=\frac{\sum_{\gamma \in \Gamma_{M}} \gamma^{k \alpha_{o}-\mu}}{\left|\Gamma_{M}\right|} \int_{\mathcal{M}_{\xi}} \operatorname{Todd}\left(\mathcal{M}_{\xi}\right) e^{\frac{1}{2 \pi}\left(k \Omega_{\xi}-\left\langle k \xi-\mu, \omega_{\xi}\right\rangle\right)} .
$$

The term $\frac{\sum_{\gamma \in \Gamma_{M}} \gamma^{k \alpha_{o}-\mu}}{\left|\Gamma_{M}\right|}$ is equal to 1 when $(\mu, k)$ belongs to the lattice $\Xi(M, L)$ (see $(1.47)$ ), and is equal to 0 in the other cases. From (1.60) we see that $P_{S}$ is a periodic polynomial of degree less than $\frac{\operatorname{dim}(\bar{S} / T)}{2}$, and for $S=S_{\max }$ we have on $\Xi(M, L)$ 


$$
P_{\max }(\mu, k)=\frac{1}{(2 \pi)^{d}} \int_{\mathcal{M}_{\xi}} \frac{\left(k \Omega_{\xi}-\left\langle k \xi-\mu, \omega_{\xi}\right\rangle\right)^{d}}{d !}+O(d-1)
$$

where $d=\frac{\operatorname{dim} \mathcal{M}_{\xi}}{2}$ and $O(d-1)$ denotes a polynomial of degree less than $d-1$. If we use the polynomial $\mathrm{DH}_{\mathfrak{c}}$ defined in Section 1.2 we can conclude our computations with the following

Proposition 1.3.22 The map $\mathrm{m}_{\mathfrak{c}}$ is a periodic polynomial of degree $d=$ $\frac{\operatorname{dim} \mathcal{M}_{\xi}}{2}$ supported on $\Xi(M, L)$. For $(\mu, k) \in \Xi(M, L)$ we have

$$
\mathrm{m}_{\mathfrak{c}}(\mu, k)=\left|\Gamma_{M}\right| \frac{k^{d}}{(2 \pi)^{d}} \mathrm{DH}_{\mathfrak{c}}\left(\frac{\mu}{k}\right)+O(d-1),
$$

where $O(d-1)$ means a periodic polynomial of degree less than $d-1$.

\subsubsection{Wall-crossing formulas for the $\mathrm{m}_{\mathfrak{c}}$}

Let $\mathfrak{c}_{+}$and $\mathfrak{c}_{-}$be two adjacent connected component of regular values of $\Phi$ separated by an hyperplane $\Delta$. The aim of this section is to compute the periodic polynomial $\mathrm{m}_{\mathfrak{c}_{+}}-\mathrm{m}_{\mathfrak{c}_{-}}$.

We consider two points $\xi_{ \pm} \in \mathfrak{c}_{ \pm}$such that $\xi=\frac{1}{2}\left(\xi_{+}+\xi_{-}\right)$belongs to the relative interior of $\overline{\mathfrak{c}_{+}} \cap \overline{\mathfrak{c}_{-}}$in $\Delta$. We suppose furthermore that $\xi^{+}-\xi^{-}$is orthogonal to $\Delta$. Using the identification $\mathfrak{t}^{*} \simeq \mathfrak{t}$ given by the scalar product the vector $\gamma=\frac{1}{2}\left(\xi_{+}-\xi_{-}\right)$, seen as a vector of $\mathfrak{t}_{\Delta}$, belongs ${ }^{4}$ to $\mathbb{R}^{>0} \beta$. We noticed in Section 1.2.4 that for all $m \in \Phi^{-1}(\xi)$ the stabilizer $\mathfrak{t}_{m}$ is either equal to $\mathfrak{t}_{\Delta}$ or to $\{0\}$. Then there exists an open $T$-invariant neighborhood $\mathcal{U}$ of $\Phi^{-1}(\xi)$ in $M$ such that for all $m \in \overline{\mathcal{U}}$ either $\mathfrak{t}_{m}:=\{0\}$, or $\mathfrak{t}_{m}=\mathfrak{t}_{\Delta}$ and $\Phi(m) \in \Delta$.

One see easily that the couple $(\mathcal{U}, \xi)$ is good and the second point of Proposition 1.3.10 tells us that

$$
R R_{\mathcal{U}}^{\xi}(M,-)=R R_{\mathcal{U}}^{\xi_{-}}(M,-)=R R_{\mathcal{U}}^{\xi_{+}}(M,-)
$$

when $\xi_{ \pm}$are close enough to $\xi$. Since $\mathcal{U} \cap \operatorname{Cr}\left(\|\Phi-\xi\|^{2}\right)=\Phi^{-1}(\xi)$ we have $R R_{\mathcal{U}}^{\xi}(M,-)=R R_{0}^{\xi}(M,-)$. If $\xi_{ \pm}$are close enough to $\xi$ we have

$$
\mathcal{U} \cap \operatorname{Cr}\left(\left\|\Phi-\xi_{ \pm}\right\|^{2}\right)=\Phi^{-1}\left(\xi_{ \pm}\right) \bigcup M^{\gamma} \cap \Phi^{-1}(\xi) .
$$

The former decomposition is due to (1.39) and to the fact that the stabiliser of $\mathfrak{t}$ on $\mathcal{U}$ are either equal to $\mathfrak{t}_{\Delta}$ or to $\{0\}$. Notice that $\xi_{-}+\gamma=\xi_{+}+\gamma=\xi$. The decomposition (1.64) gives

$$
R R_{\mathcal{U}}^{\xi_{ \pm}}(M,-)=R R_{0}^{\xi_{ \pm}}(M,-)+R R_{\mp \gamma}^{\xi_{ \pm}}(M,-)
$$

where $R R_{\gamma}^{\xi_{-}}(M,-)$ (resp. $\left.R R_{-\gamma}^{\xi_{+}}(M,-)\right)$ is the Riemann-Roch character localized on $M^{\gamma} \cap \Phi^{-1}(\xi)$ by the vector field $\mathcal{H}-\left(\xi_{-}\right)_{M}\left(\operatorname{resp} . \mathcal{H}-\left(\xi_{+}\right)_{M}\right)$. Now (1.63) and (1.65) prove the following

\footnotetext{
${ }^{4} \beta$ is the primitive vector of $\mathfrak{t}_{\Delta} \cap \Lambda$ pointing out of $\mathfrak{c}_{-}$
} 
Proposition 1.3.23 If $\xi_{ \pm}$are close enough to $\Delta$, we have

$$
R R_{0}^{\xi_{+}}(M,-)-R R_{0}^{\xi_{-}}(M,-)=R R_{\gamma}^{\xi_{-}}(M,-)-R R_{-\gamma}^{\xi_{+}}(M,-) .
$$

We know from Proposition 1.3.18 that $\mathrm{m}_{\mathfrak{c}_{ \pm}}(\mu, k)$ is equal to the $\mu$ multiplicity of $T$ in $R R_{0}^{\xi_{ \pm}}\left(M, L^{\otimes k}\right)$. Hence $\mathrm{m}_{\mathfrak{c}_{+}}(\mu, k)-\mathrm{m}_{\mathfrak{c}_{-}}(\mu, k)$ is equal to the $\mu$-multiplicity of $T$ in $R R_{\gamma}^{\xi_{-}}\left(M, L^{\otimes k}\right)-R R_{-\gamma}^{\xi_{+}}\left(M, L^{\otimes k}\right)$.

Let $N_{\Delta}$ be the normal bundle of $M^{T_{\Delta}}$ in $M$, and let $\left[\wedge_{\mathbb{C}}^{\bullet} \overline{N_{\Delta}}\right]_{ \pm \beta}^{-1}$ be the polarized inverses of $\wedge_{\mathbb{C}}^{\bullet} \overline{N_{\Delta}}$ (see Proposition 1.3.13). Since $\xi=\xi_{+}-\gamma=\xi_{-}+\gamma$ and $\gamma \in \mathbb{R}^{>0} \beta$, the localization (1.44) gives

$$
\begin{aligned}
& R R_{\gamma}^{\xi_{-}}\left(M, L^{\otimes k}\right)=\sum_{Z \in \mathcal{F}} R R_{0}^{\xi}\left(Z,\left.L^{\otimes k}\right|_{Z} \otimes\left[\wedge_{\mathbb{C}}^{\bullet} N_{Z}\right]_{\beta}^{-1}\right) \\
& R R_{-\gamma}^{\xi_{+}}\left(M, L^{\otimes k}\right)=\sum_{Z \in \mathcal{F}} R R_{0}^{\xi}\left(Z,\left.L^{\otimes k}\right|_{Z} \otimes\left[\wedge_{\mathbb{C}}^{\bullet} \bar{N}_{Z}\right]_{-\beta}^{-1}\right)
\end{aligned}
$$

Finally $\mathrm{m}_{\mathfrak{c}_{+}}(\mu, k)-\mathrm{m}_{\mathfrak{c}_{-}}(\mu, k)=\sum_{Z \in \mathcal{F}} \mathcal{A}_{Z}(\mu, k)$ where $\mathcal{A}_{Z}(\mu, k)$ is equal to the $\mu$-multiplicity of $T$ in

$$
R R_{0}^{\xi}\left(Z,\left.L^{\otimes k}\right|_{Z} \otimes\left[\wedge_{\mathbb{C}}^{\bullet} \overline{N_{Z}}\right]_{-\beta}^{-1}\right)-R R_{0}^{\xi}\left(Z,\left.L^{\otimes k}\right|_{Z} \otimes\left[\wedge_{\mathbb{C}} \overline{N_{Z}}\right]_{\beta}^{-1}\right)
$$

Let $\beta^{\prime} \in \mathfrak{t}_{\Delta}^{*} \cap \Lambda^{*}$ which is defined by the relation $\left\langle\beta^{\prime}, \beta\right\rangle=2 \pi$, so that $\Lambda_{\mathfrak{t}_{\Delta}}^{*}=\mathbb{Z} \beta^{\prime}$. Concerning the $T_{\Delta}$-weights we have

1. The $T_{\Delta}$-weight on $\left.L^{\otimes k}\right|_{Z}$ is equal to $k r_{\Delta} \beta^{\prime}$.

2. The $T_{\Delta}$-weight on $\operatorname{det}\left(N_{Z}^{+, \pm \beta}\right)$ is $\pm s_{Z}^{ \pm} \beta^{\prime}$ where $s_{Z}^{ \pm} \in \mathbb{N}$ is the absolute value of the trace of $\frac{1}{2 \pi} \mathcal{L}_{\beta}$ on $N_{Z}^{+, \pm \gamma}$.

3. The $T_{\Delta}$-weights on $S^{>0}\left(N_{Z}^{+, \beta}\right)$ (resp. $S^{>0}\left(N_{Z}^{+,-\beta}\right)$ ) are of the form $p \beta^{\prime}$ with $p>0$ (resp. $p<0)$.

Now Lemma 1.3.14 shows that if a weight $\mu$ occurs in $R R_{0}^{\xi}\left(Z,\left.L^{\otimes k}\right|_{Z} \otimes\left[\wedge_{\mathbb{C}} \overline{N_{Z}}\right]_{\beta}^{-1}\right)$ we have $i^{*}(\mu)=\left(k r_{\Delta}+s_{Z}^{+}+p\right) \beta^{\prime}$ with $p \geq 0$, and then

$$
\frac{\langle\mu, \beta\rangle}{2 \pi}-k r_{\Delta} \geq s_{Z}^{+} .
$$

Similarly, if a weight $\mu$ occurs in $R R_{0}^{\xi}\left(Z,\left.L^{\otimes k}\right|_{Z} \otimes\left[\wedge_{\mathbb{C}} \bar{N}_{Z}\right]_{-\beta}^{-1}\right)$ we have

$$
\frac{\langle\mu, \beta\rangle}{2 \pi}-k r_{\Delta} \leq-s_{Z}^{-} .
$$

Finally, $\mathcal{A}_{Z}(\mu, k)=0$ when $-s_{Z}^{-}<\frac{\langle\mu, \beta\rangle}{2 \pi}-k r_{\Delta}<s_{Z}^{+}$. We have proved the following 
Theorem 1.3.24 Let $s^{ \pm}=\inf _{Z} s_{Z}^{ \pm}$where the infimum is taken over the connected components $Z$ of $M^{T_{\Delta}}$ for which $\overline{\mathfrak{c}_{+}} \cap \overline{\mathfrak{c}_{-}} \subset \Phi(Z)$. For every $(\mu, k) \in \Lambda^{*} \times \mathbb{Z}$, we have $\mathrm{m}_{\mathfrak{c}_{+}}(\mu, k)=\mathrm{m}_{\mathfrak{c}_{-}}(\mu, k)$ if

$$
-s^{-}<\frac{\langle\mu, \beta\rangle}{2 \pi}-k r_{\Delta}<s^{+} .
$$

Sometimes the inequalities (1.67) are optimal.

Proposition 1.3.25 • Consider the connected components $Z \in \mathcal{F}$ for which $s_{Z}^{+}$is minimal. Among them consider the subset $\mathcal{F}_{+}$where $\operatorname{dim}(Z)$ is maximal. If the integers $\operatorname{rk}_{\mathbb{C}}\left(N_{Z}^{+, \beta}\right), Z \in \mathcal{F}_{+}$have the same parity, then the condition “ $\frac{\langle\mu, \beta\rangle}{2 \pi}-k r_{\Delta}<s^{+}$" is optimal in (1.67).

- In the same way, consider the connected components $Z \in \mathcal{F}$ for which $s_{Z}^{-}$ is minimal. Among them consider the subset $\mathcal{F}_{-}$where $\operatorname{dim}(Z)$ is maximal. If the integers $\operatorname{rk}_{\mathbb{C}}\left(N_{Z}^{+, \beta}\right), Z \in \mathcal{F}_{+}$have the same parity, then the condition " $-s^{-}<\frac{\langle\mu, \beta\rangle}{2 \pi}-k r_{\Delta}$ " is optimal in (1.67).

Remark 1.3.26 The last Proposition applies when there is a unique connected component $Z$ of $M^{T_{\Delta}}$ for which $\overline{\mathfrak{c}_{+}} \cap \overline{\mathfrak{c}_{-}} \subset \Phi(Z)$.

Proof. We consider only the first point since the other point works similarly. We restrict our attention to the couples $(\mu, k)$ such that $\frac{\langle\mu, \beta\rangle}{2 \pi}-k r_{\Delta}=s^{+}$. They are of the form

$$
\mu=\left(k r_{\Delta}+s^{+}\right) \beta^{\prime}+\mu_{2}
$$

with $\mu_{2} \in \Lambda_{\mathfrak{t} / \mathfrak{t}_{\Delta}}^{*}$. Let us denote $D\left(\mu_{2}, k\right)$ the restriction of $\mathrm{m}_{\mathfrak{c}_{+}}(\mu, k)-$ $\mathrm{m}_{\mathfrak{c}_{-}}(\mu, k)$ to the set of couples $(\mu, k)$ parametrized by (1.68). We want to prove that $D\left(\mu_{2}, k\right)$ is not identically equal to zero.

From the previous discussion one knows that

$$
D\left(\mu_{2}, k\right)=\sum_{Z, s_{Z}^{+}=s^{+}}(-1)^{\mathrm{rk} c\left(N_{Z}^{+, \beta}\right)} D_{Z}\left(\mu_{2}, k\right),
$$

where $D_{Z}\left(\mu_{2}, k\right)$ is the $\mu$-multiplicity of $T$ in

$$
R R_{0}^{\xi}\left(Z,\left.L^{\otimes k}\right|_{Z} \otimes \operatorname{det}\left(N_{Z}^{+, \beta}\right)\right) .
$$

Let us make few remarks concerning the maps $R R_{0}^{\xi}(Z,-): \mathbf{K}_{T}(Z) \rightarrow$ $R^{-\infty}(T)$. Since $T_{\Delta}$ acts trivially on $Z$, the decomposition $T=T / T_{\Delta} \times T_{\Delta}$ induces a canonical isomorphism $\mathbf{K}_{T}(Z) \simeq \mathbf{K}_{T / T_{\Delta}}(Z) \otimes R\left(T_{\Delta}\right)$ : i.e. every $T$-equivariant vector bundle $E \rightarrow Z$ decomposes as

$$
E=\sum_{\mu_{1} \in \mathbb{Z} \beta^{\prime}} E^{\mu_{1}} \otimes \mathbb{C}_{\mu_{1}} .
$$

Here each $E^{\mu_{1}}$ is a $T / T_{\Delta}$-equivariant vector bundle on $Z$ and $\mathbb{C}_{\mu_{1}}$ denotes the one dimensional $T_{\Delta}$-representation associated to $\mu_{1} \in \Lambda_{\mathfrak{t}_{\Delta}}^{*}$. 
For every $T$-equivariant vector bundle $E \rightarrow Z$, the character $R R_{0}^{\xi}(Z, E)$ is equal to the $T$-equivariant index of the $T$-transversally elliptic symbol $\operatorname{Thom}_{\xi}(\mathcal{V}) \otimes p^{*}(E)$, where $\mathcal{V}$ is a small neighborhood of $\Phi^{-1}(\xi) \cap Z$ in $Z$ (see Definition 1.3.9). Since the $T_{\Delta}$ action is trivial on $Z$ the symbol $\operatorname{Thom}_{\xi}(\mathcal{V})$ is also $T / T_{\Delta}$ transversally elliptic and the action of $T_{\Delta}$ is trivial on it. We have then

$$
R R_{0}^{\xi}(Z, E)=\sum_{\mu_{1} \in \mathbb{Z} \beta^{\prime}} R R_{0}^{\xi}\left(Z, E^{\mu_{1}}\right) \otimes \mathbb{C}_{\mu_{1}}
$$

where the character $R R_{0}^{\xi}\left(Z, E^{\mu_{1}}\right) \in R^{-\infty}\left(T / T_{\Delta}\right)$ is computed by Theorem 1.46 applied to the Hamiltonian $T / T_{\Delta}$-manifold $Z$. For every $T$-vector bundle $E \rightarrow Z$ we define the familly $\mathcal{E}_{\xi}^{\mu_{1}, \mu_{2}}, \mu_{1} \in \mathbb{Z} \beta^{\prime}, \mu_{2} \in \Lambda_{\mathfrak{t} / \mathfrak{t}_{\Delta}}^{*}$ of orbifold vector bundles over the reduced space $\mathcal{Z}_{\xi}=Z \cap \Phi^{-1}(\xi) /\left(T / T_{\Delta}\right)$ by

$$
\mathcal{E}_{\xi}^{\mu_{1}, \mu_{2}}:=\left.\left(E^{\mu_{1}} \otimes \mathbb{C}_{-\mu_{2}}\right)\right|_{\Phi^{-1}(\xi) \cap Z} /\left(T / T_{\Delta}\right) .
$$

Finally (1.46) and (1.71) give the following

$$
\begin{aligned}
R R_{0}^{\xi}(Z, E) & =\sum_{\mu_{1} \in \mathbb{Z} \beta^{\prime}} \sum_{\mu_{2} \in \Lambda_{\mathfrak{t} / t_{\Delta}}^{*}} R R\left(\mathcal{Z}_{\xi}, \mathcal{E}_{\xi}^{\mu_{1}, \mu_{2}}\right) \otimes \underbrace{\mathbb{C}_{\mu_{1}}}_{\in R\left(T_{\Delta}\right)} \otimes \underbrace{\mathbb{C}_{\mu_{2}}}_{\in R\left(T / T_{\Delta}\right)} \\
& =\sum_{\mu \in \Lambda^{*}} R R\left(\mathcal{Z}_{\xi}, \mathcal{E}_{\xi}^{\mu_{1}, \mu_{2}}\right) \mathbb{C}_{\mu} .
\end{aligned}
$$

In (1.73) we write $\mu \in \Lambda^{*}$ as a sum of $\mu_{1} \in \mathbb{Z} \beta^{\prime}$ with $\mu_{2} \in \Lambda_{\mathfrak{t} / \mathfrak{t}_{\Delta}}^{*}$ so that $\mathbb{C}_{\mu} \in R(T)$ is equal to the tensor product $\mathbb{C}_{\mu_{1}} \otimes \mathbb{C}_{\mu_{1}}$.

When the vector bundle $E \rightarrow Z$ is the line bundle $\mathbb{L}:=\left.L^{\otimes k}\right|_{Z} \otimes \operatorname{det}\left(N_{Z}^{+, \beta}\right)$ we have $\mathbb{L}=\mathbb{L}^{j \beta^{\prime}} \otimes \mathbb{C}_{j \beta^{\prime}}$ for $j=k r_{\Delta}+s^{+}$. Finally, we have

$$
D_{Z}\left(\mu_{2}, k\right)=R R\left(\mathcal{Z}_{\xi}, \mathbb{L}_{\xi}^{\left(k r_{\Delta}+s^{+}\right) \beta^{\prime}, \mu_{2}}\right) .
$$

Now we use the results of Section 1.3.4 to study the map

$$
D_{Z}: \Lambda_{\mathfrak{t} / \mathfrak{t}_{\Delta}}^{*} \times \mathbb{Z} \longrightarrow \mathbb{Z}
$$

Let $\Gamma_{Z} \subset T / T_{\Delta}$ be the generic stabiliser of $T / T_{\Delta}$ on a component $Z$. Let $\alpha_{Z}, \delta_{Z} \in \Lambda_{\mathfrak{t} / \mathfrak{t}_{\Delta}}^{*}$ such that the action of $\Gamma_{Z}$ on the fibers of $L_{\mid Z}$ and $\operatorname{det}\left(N_{Z}^{+, \beta}\right)$ are respectively $t \rightarrow t^{\alpha_{Z}}$ and $t \rightarrow t^{\delta_{Z}}$. After Remark 1.3.17 we know that the map (1.74) is supported on the subset

$$
\Xi_{Z}:=\left\{\left(\mu_{2}, k\right) \in \Lambda_{\mathfrak{t} / \mathfrak{t}_{\Delta}}^{*} \times \mathbb{Z} \mid t^{k \alpha_{Z}+\delta_{Z}+\mu_{2}}=1, \forall t \in \Gamma_{Z}\right\} .
$$

The only difference with the computations done in Section 1.3.4 is the line bundle $\operatorname{det}\left(N_{Z}^{+, \beta}\right)$. But this do not change the global behaviour of the map (1.74) on $\Xi_{Z}$ : it is a periodic polynomial map of degree $d_{Z}=\operatorname{dim}\left(\mathcal{Z}_{\xi}\right) / 2$ and we have 


$$
D_{Z}\left(\mu_{2}, k\right)=\frac{1}{(2 \pi)^{d_{Z}}} \int_{\mathcal{Z}_{\xi}} \frac{\left(k \Omega_{\mathcal{Z}_{\xi}}-\left\langle k \xi-\mu_{2}, \omega_{\mathcal{Z}_{\xi}}\right\rangle\right)^{d_{Z}}}{d_{Z} !}+O\left(d_{Z}-1\right)
$$

for all $\left(\mu_{2}, k\right) \in \Xi_{Z}$.

Suppose now that all the signs $(-1)^{\mathrm{rk}_{\mathbb{C}}\left(N_{Z}^{+, \beta}\right)}$ coincide when $Z \in \mathcal{F}_{+}$. From (1.69), we get that $D\left(\mu_{2}, k\right)$ does not vanish for large values of $\left(\mu_{2}, k\right)$.

\subsection{Multiplicities of group representations}

Let $K$ be a semi-simple compact Lie group with Lie algebra $\mathfrak{k}$, and let $T$ be a maximal torus in $K$ with Lie algebra $\mathfrak{t}$. In this section we denote $(-,-)$ the scalar product on $\mathfrak{k}$ induced by the Killing form, and we keep the same notation for the induced scalar products on $\mathfrak{t}^{*}$ and on $\mathfrak{t}$.

Let $\Lambda^{*} \subset \mathfrak{t}^{*}$ be the weight lattice, and let $\mathfrak{R} \subset \Lambda^{*}$ be the set of roots for the action of $T$ on $\mathfrak{k} \otimes \mathbb{C}$ : we denote $\Lambda_{\mathfrak{R}}^{*}$ the sub-lattice of $\Lambda^{*}$ generated by $\mathfrak{R}$. We choose a system of positive roots $\mathfrak{R}^{+} \subset \mathfrak{R}$, and we denote $\mathfrak{t}_{+}^{*}$ the corresponding Weyl chamber.

The irreducible representations of $K$ are parametrized by the set $\Lambda_{+}^{*}=$ $\Lambda^{*} \cap \mathfrak{t}_{+}^{*}$. For $\lambda \in \Lambda_{+}^{*}$ we denote by $V_{\lambda}$ the irreducible representation of $K$ with heighest weight $\lambda$. Here we are interested in the $T$-multiplicities in $\left.V_{\lambda}\right|_{T}$. Let $\mathrm{m}: \Lambda^{*} \times \Lambda_{+}^{*} \rightarrow \mathbb{N}$ be the map defined by

$$
\left.V_{\lambda}\right|_{T}=\sum_{\mu \in \Lambda^{*}} \mathrm{~m}(\mu, \lambda) \mathbb{C}_{\mu}
$$

for every $\lambda \in \Lambda_{+}^{*}$.

Definition 1.4.1 For every $\lambda \in \Lambda_{+}^{*}$, we denote $\mathrm{m}^{\lambda}: \Lambda^{*} \times \mathbb{Z}^{>0} \rightarrow \mathbb{N}$ the map defined by $\mathrm{m}^{\lambda}(\mu, k)=\mathrm{m}(\mu, k \lambda)$. So $\mathrm{m}^{\lambda}(\mu, k)$ is equal to the multiplicity of $\mathbb{C}_{\mu}$ in $\left.V_{k \lambda}\right|_{T}$.

\subsubsection{Borel-Weil Theorem}

First we recall the realization of the $K$-representation $V_{\lambda}$ given by the BorelWeil Theorem. The coadjoint orbit $K \cdot \lambda$ is equipped with the Kirillov-KostantSouriau symplectic form $\Omega$ which is defined by:

$$
\Omega\left(X_{M}, Y_{M}\right)_{m}=\langle m,[X, Y]\rangle, \quad \text { for } \quad m \in K \cdot \lambda \quad \text { and } \quad X, Y \in \mathfrak{k} .
$$

The action of $K$ on $K \cdot \lambda$ is Hamiltonian with moment map $K \cdot \lambda \hookrightarrow \mathfrak{k}^{*}$ equal to the inclusion. The action of $T$ on $K \cdot \lambda$ is also Hamiltonian with moment map $\Phi: K \cdot \lambda \rightarrow \mathfrak{t}^{*}$ equal to the composition of the inclusion $K \cdot \lambda \hookrightarrow \mathfrak{k}^{*}$ with the projection map $\mathfrak{k}^{*} \rightarrow \mathfrak{t}^{*}$.

There exists a unique $K$-invariant complex structure on $K \cdot \lambda$ compatible with the symplectic form. In this situation the Kostant-Souriau prequantum line bundle over $K \cdot \lambda$ is 


$$
\mathbb{C}_{[\lambda]}=K \times_{K_{\lambda}} \mathbb{C}_{\lambda} .
$$

Here we use the canonical identification $K / K_{\lambda} \simeq K \cdot \lambda,[k] \mapsto k \cdot \lambda$, where $K_{\lambda}$ is the stabilizer of $\lambda$ in $K$. The line bundle $\mathbb{C}_{[\lambda]}$ over the complex manifold $K \cdot \lambda$ carries a canonical holomorphic structure. If one work with the symplectic form $k \Omega$, for an integer $k \geq 1$, the corresponding Kostant-Souriau prequantum line bundle is $\mathbb{C}_{[\lambda]}^{\otimes k}=K \times_{K_{\lambda}} \mathbb{C}_{k \lambda}=\mathbb{C}_{[k \lambda]}$.

Let $\mathcal{H}^{q}\left(K \cdot \lambda, \mathbb{C}_{[\lambda]}^{\otimes k}\right)$ be $q$ th cohomology group of the sheaf of holomorphic section of $\mathbb{C}_{[\lambda]}^{\otimes k}$ over $K \cdot \lambda$. The Borel-Weil Theorem tells us that

$$
\mathcal{H}^{0}\left(K \cdot \lambda, \mathbb{C}_{[\lambda]}^{\otimes k}\right)=V_{k \lambda}
$$

and

$$
\mathcal{H}^{q}\left(K \cdot \lambda, \mathbb{C}_{[\lambda]}^{\otimes k}\right)=0 \quad \text { for } \quad q \geq 1 .
$$

If $R R^{K}(K \cdot \lambda,-): \mathbf{K}_{K}(K \cdot \lambda) \rightarrow R(K)$ is the $K$-equivariant Riemann-Roch character defined by the compatible complex structure, (1.79) and (1.80) give

$$
R R^{K}\left(K \cdot \lambda, \mathbb{C}_{[\lambda]}^{\otimes k}\right)=V_{k \lambda} \quad \text { in } \quad R(K) .
$$

Now if we denote by $R R(K \cdot \lambda,-): \mathbf{K}_{T}(K \cdot \lambda) \rightarrow R(T)$ the $T$-equivariant Riemann-Roch character, we have $\left.V_{k \lambda}\right|_{T}=R R\left(K \cdot \lambda, \mathbb{C}_{[\lambda]}^{\otimes k}\right)$. The multiplicity fonction $\mathrm{m}^{\lambda}: \Lambda_{+}^{*} \times \mathbb{N}^{*} \rightarrow \mathbb{N}$ is characterized by the relation

$$
R R\left(K \cdot \lambda, \mathbb{C}_{[\lambda]}^{\otimes k}\right)=\sum_{\mu \in \Lambda^{*}} \mathrm{~m}^{\lambda}(\mu, k) \mathbb{C}_{\mu}, \quad \text { in } R(T),
$$

for $k \geq 1$.

The sub-lattice $\Lambda_{\mathfrak{R}}^{*}$ of $\Lambda^{*}$ generated by the roots is characterized by the (finite) center $Z(K)$ of $K$ as follows. For $\alpha \in \Lambda^{*}$ we have

$$
\lambda \in \Lambda_{\mathfrak{R}}^{*} \Longleftrightarrow t^{\lambda}=1, \forall t \in Z(K),
$$

and for $t \in T$ we have $t \in Z(K) \Longleftrightarrow t^{\lambda}=1, \forall \lambda \in \Lambda_{\mathfrak{R}}^{*}$. The finite abelian group $\Lambda^{*} / \Lambda_{\mathfrak{R}}^{*}$ is then naturally identified with the dual of $Z(K)$. We have the following well-known fact.

Lemma 1.4.2 The mutiplicity map $\mathrm{m}^{\lambda}$ is supported on the sub-lattice $\Xi_{\lambda}=$ $\left\{(\mu, k) \in \Lambda^{*} \times \mathbb{Z} \mid \mu-k \lambda \in \Lambda_{\mathfrak{R}}^{*}\right\}$.

Proof. The center $Z(K)$ of $K$ acts trivially on $K \cdot \lambda$ and with the character $t \in Z(K) \mapsto t^{k \lambda}$ on the fibers of the line bundle $\mathbb{C}_{[\lambda]}^{\otimes k}$. Since $\mathrm{m}^{\lambda}(\mu, k)$ is equal to the dimension of the $T$-invariant subspace of $R R\left(K \cdot \lambda, \mathbb{C}_{[\lambda]}^{\otimes k}\right) \otimes \mathbb{C}_{-\mu}$, we have following Lemma 1.3.17 that $\mathrm{m}^{\lambda}(\mu, k) \neq 0$ only if $t^{\mu-k \lambda}=1, \forall t \in Z(K)$. We conclude then with (1.83).

In this section we study the periodic polynomials 


$$
\mathrm{m}_{\mathfrak{c}}^{\lambda}: \Lambda^{*} \times \mathbb{Z} \longrightarrow \mathbb{Z}
$$

defined for every connected component $\mathfrak{c} \subset \mathfrak{t}^{*}$ of regular values of the moment map $\Phi: K \cdot \lambda \rightarrow \mathfrak{t}^{*}$. We know that $\mathrm{m}_{\mathfrak{c}}^{\lambda}$ is also supported on the sub-lattice $\Xi_{\lambda}$ (see Section 1.3.3).

In order to apply Theorem 1.3.24 to the periodic polynomials $\mathrm{m}_{\mathfrak{c}}^{\lambda}$, we have to compute the critical values of the moment map $\Phi: K \cdot \lambda \rightarrow \mathfrak{t}^{*}$.

\subsubsection{Critical points of $\Phi: K \cdot \lambda \rightarrow \mathfrak{t}^{*}$}

Let $\left\{\alpha_{1}, \cdots, \alpha_{\operatorname{dim} T}\right\}$ be the simple roots of the set $\mathfrak{R}_{+}$of positive weights. The fundamental weights $\varpi_{k}, 1 \leq k \leq \operatorname{dim} T$ are defined by the conditions

$$
2 \frac{\left(\varpi_{i}, \alpha_{j}\right)}{\left|\alpha_{j}\right|^{2}}=\delta_{i, j} \quad \text { for all } \quad 1 \leq i, j \leq \operatorname{dim} T .
$$

Recall that the fundamental weights generate the lattice $\Lambda_{\text {alg }}^{*}$ of algebraic integral element of $\mathfrak{t}^{*}$. We have $\Lambda^{*} \subset \Lambda_{\text {alg }}^{*}$ and equality holds only if $K$ is simply-connected.

Let $W$ be the Weyl group of $(K, T)$. We will look at

$$
\mathcal{G}=\left\{\sigma \cdot \varpi_{i} \mid \sigma \in W, 1 \leq i \leq \operatorname{dim} T\right\} .
$$

as a subset of $\mathfrak{t}$ modulo the identification $\mathfrak{t} \simeq \mathfrak{t}^{*}$ given by the scalar product. The singular points of $\Phi$ have the following nice description. This result first appeared in Heckman's Thesis [22].

Proposition 1.4.3 ([22, 17]) The critical points of $\Phi: K \cdot \lambda \rightarrow \mathfrak{t}^{*}$ is the union of the fixed points set $(K \cdot \lambda)^{\beta}, \beta \in \mathcal{G}$. For each $\beta \in \mathcal{G}$ we have

$$
(K \cdot \lambda)^{\beta}=\bigcup_{\sigma \in W} K^{\beta} \cdot \sigma \lambda,
$$

where $K^{\beta}$ is the stabilizer subgroup of $\beta$ in $K$.

The fixed points of the action of $T$ on $K \cdot \lambda$ characterize the image of $\Phi$ completely: $\Phi(K \cdot \lambda)$ is the convex polytope

$$
\operatorname{conv}(W \cdot \lambda):=\text { convex hull of } W \cdot \lambda .
$$

This result was first proved by Kostant [25]. This is particular case of the convexity theorem of Atiyah, Guillemin and Sternberg [2, 18]. From Proposition 1.4.3, we know that the singular values of $\Phi: K \cdot \lambda \rightarrow \mathfrak{t}^{*}$ are the convex polytopes

$$
\operatorname{conv}\left(W^{\beta} \cdot \sigma \lambda\right), \quad \beta \in \mathcal{F}, \sigma \in W / W^{\beta},
$$

where $W^{\beta}$ is the stabilizer ${ }^{5}$ of $\beta$ in $W$, i.e. $W^{\beta}$ is the Weyl group of $\left(K^{\beta}, T\right)$. Each convex polytope $\operatorname{conv}\left(W^{\beta} \cdot \sigma \lambda\right)$ lies in the hyperplane

${ }^{5}$ When $\beta=\varpi_{i}$, we denote $W^{i}$ the stabilizer of $\varpi_{i}$ in $W$. 


$$
\Delta_{\beta, \sigma}=\left\{\xi \in \mathfrak{t}^{*} \mid(\xi-\sigma \lambda, \beta)=0\right\} .
$$

We will use the following

Lemma 1.4.4 $\bullet K^{\beta} \cdot \sigma \lambda=K^{\beta} \cdot \sigma^{\prime} \lambda$ if and only if $\sigma \lambda \in W^{\beta} \sigma^{\prime} \lambda$,

- $\operatorname{conv}\left(W^{\beta} \cdot \sigma \lambda\right) \cap \operatorname{conv}\left(W^{\beta} \cdot \sigma^{\prime} \lambda\right) \neq \emptyset$ if and only if $\Delta_{\beta, \sigma}=\Delta_{\beta, \sigma^{\prime}}$.

Proof. The first point follows from the fact that the intersection of a coadjoint orbit $K^{\beta} \cdot \mu, \mu \in \mathfrak{t}^{*}$ with $\mathfrak{t}^{*}$ is equal to $W^{\beta} \cdot \mu$.

It is sufficient to prove the second point for $\beta=\varpi_{i}$. The half-line $\mathbb{R}^{>0} \varpi_{i}$ is an edge of the Weyl chamber. It is well known that the following vector subspaces coincides:

- the line $\mathbb{R} \varpi_{i}$,

- the vector sub-space of $K^{\varpi_{i}}$-invariant element of $\mathfrak{k}^{*}$,

- the vector sub-space of $W^{i}$-invariant element of $\mathfrak{t}^{*}$.

Each convex polytope $\operatorname{conv}\left(W^{i} \cdot \sigma \lambda\right)$ contains the $W^{i}$-invariant element

$$
\frac{1}{\left|W^{i}\right|} \sum_{\tau \in W^{i}} \tau \cdot \sigma \lambda
$$

which is equal to the intersection of the hyperplane $\Delta_{\beta, \sigma}$ with the line $\mathbb{R} \varpi_{i}$. Hence, if $\Delta_{\beta, \sigma}=\Delta_{\beta, \sigma^{\prime}}$, the intersection $\operatorname{conv}\left(W^{\beta} \cdot \sigma \lambda\right) \cap \operatorname{conv}\left(W^{\beta} \cdot \sigma^{\prime} \lambda\right)$ contains a $W^{i}$-invariant element, and then is not empty.

Definition 1.4.5 An element $\lambda \in \Lambda_{+}^{*}$ is generic if for every fundamental root $\varpi_{i}$ and any $\sigma, \sigma^{\prime} \in W$, we have

$$
\Delta_{\beta, \sigma} \neq \Delta_{\beta, \sigma}
$$

each times the submanifolds $K^{\beta} \cdot \sigma \lambda$ and $K^{\beta} \cdot \sigma^{\prime} \lambda$ are not equal.

This condition of genericity imposes that $\left(\sigma \lambda, \varpi_{i}\right) \neq\left(\sigma^{\prime} \lambda, \varpi_{i}\right)$ when $\sigma \lambda \notin$ $W^{i} \sigma^{\prime} \lambda$.

Example 1.4.6 Consider the case of SU(4). Take the coadjoint orbit trough $\lambda=(2,1,-1,-2)$, and $\sigma, \sigma^{\prime}$ such that $\sigma \lambda=(2,-2,1,-1)$ and $\sigma^{\prime} \lambda=$ $(1,-1,2,-2)$. Take the fundamental weight $\varpi_{2}=\frac{1}{2}(1,1,-1,-1)$. In this case $\lambda$ is not "generic" since $\sigma \lambda \notin W^{i} \sigma^{\prime} \lambda$ but $\left(\sigma \lambda, \varpi_{2}\right)=0=\left(\sigma^{\prime} \lambda, \varpi_{2}\right)$.

\subsubsection{Main theorems}

Let $\mathfrak{c}_{+}$and $\mathfrak{c}_{-}$be two adjacent connected components of regular values of $\Phi$ : $K \cdot \lambda \rightarrow \mathfrak{t}^{*}$. The intersection $\overline{\mathfrak{c}_{+}} \cap \overline{\mathfrak{c}_{+}}$is contained in an hyperplane orthogonal to $\beta \in \mathcal{F}$. 
Definition 1.4.7 Let $\mathcal{A}\left(\mathfrak{c}_{+}, \mathfrak{c}_{-}\right)$be the set of all $[\sigma] \in W / W^{\beta}$ such that the convex polytope $\operatorname{conv}\left(W^{\beta} \cdot \sigma \lambda\right)$ contains $\overline{\mathfrak{c}_{+}} \cap \overline{\mathfrak{c}_{+}}$.

The following

$$
\bigcup_{[\sigma] \in \mathcal{A}\left(\mathfrak{c}_{+}, \mathfrak{c}_{-}\right)} K^{\beta} \cdot \sigma \lambda
$$

is the union of the connected components of $(K \cdot \lambda)^{\beta}$ that intersect $\Phi^{-1}(\xi)$ when $\xi \in \overline{\mathfrak{c}_{+}} \cap \overline{\mathfrak{c}_{+}}$.

Remark 1.4.8 When $\lambda$ is a regular element of $\mathfrak{t}^{*}$, all polytopes $\operatorname{conv}\left(W^{\beta} \cdot \sigma \lambda\right)$ are of codimension 1. When $\lambda$ is "generic" (see Def. 1.4.5), the set $\mathcal{A}\left(\mathfrak{c}_{+}, \mathfrak{c}_{-}\right)$ is reduced to one element.

The multiplicity function $\mathrm{m}^{\lambda}: \Lambda^{*} \times \mathbb{Z}^{>0} \rightarrow \mathbb{N}$ is invariant under the action of the Weyl group: $\mathrm{m}^{\lambda}(\sigma \mu, k)=\mathrm{m}^{\lambda}(\mu, k)$ for every $\sigma \in W$. The set of connected component of regular values of $\Phi$ is also invariant under the action of $W$.

So, for the rest of this section we restrict our attention to case where $\mathfrak{c}_{+}$ and $\mathfrak{c}_{-}$are separated by an hyperplane orthogonal to a fundamental weight $\beta=\varpi_{i}$ : the vector $\varpi_{i}$ is pointing out of $\mathfrak{c}_{-}$. We denote $K^{i}$ the stabilizer of $\varpi_{i}$ in $K$.

Consider $[\sigma] \in \mathcal{A}\left(\mathfrak{c}_{+}, \mathfrak{c}_{-}\right)$and let $K^{i} \cdot \sigma \lambda$ be the corresponding connected component of $(K \cdot \lambda)^{\beta}$. The tangent space of $K \cdot \lambda$ at $\sigma \lambda$ is the following $K^{\sigma \lambda}$-module

$$
\mathbf{T}_{\sigma \lambda}(K \cdot \lambda)=\sum_{(\alpha, \sigma \lambda)>0} \mathfrak{k}_{\alpha}
$$

where $\mathfrak{k}_{\alpha} \subset \mathfrak{k} \otimes \mathbb{C}$ is the one-dimensional complex subspace associated to the weight $\alpha \in \mathfrak{R}$. In the same way, the tangent space of $K^{i} \cdot \sigma \lambda$ at $\sigma \lambda$ is the $K^{i} \cap K^{\sigma \lambda}$-module defined by

$$
\mathbf{T}_{\sigma \lambda}\left(K^{i} \cdot \sigma \lambda\right)=\sum_{\substack{(\alpha, \sigma \lambda)>0 \\\left(\alpha, \varpi_{i}\right)=0}} \mathfrak{k}_{\alpha}
$$

Finally the normal bundle of $K^{i} \cdot \sigma \lambda \simeq K^{i} /\left(K^{i} \cap K^{\sigma \lambda}\right)$ in $K \cdot \lambda$ is $\mathcal{N}_{\sigma, i}=$ $K^{i} \times_{K^{i} \cap K^{\sigma \lambda}} N_{\sigma, i}$ where

$$
N_{\sigma, i}=\sum_{\substack{(\alpha, \sigma \lambda)>0 \\\left(\alpha, \varpi_{i}\right) \neq 0}} \mathfrak{k}_{\alpha}
$$

For an element $\mu \in \mathfrak{t}^{*}$, we have $\mu=\sum_{i=1}^{\operatorname{dim} T}[\mu]_{k} \alpha_{k}$ where

$$
[\mu]_{k}=2 \frac{\left(\varpi_{k}, \mu\right)}{\left|\alpha_{k}\right|^{2}} \in \mathbb{R} .
$$

Note that $[\mu]_{k} \in \mathbb{Z}$ when $\mu$ belongs to the lattice $\Lambda_{\mathfrak{R}}^{*}$. 
Definition 1.4.9 For $[\sigma] \in \mathcal{A}\left(\mathfrak{c}_{+}, \mathfrak{c}_{-}\right)$we define the positive integers

$$
s_{\sigma, i}^{ \pm}= \pm \sum_{\substack{(\alpha, \sigma \lambda)>0 \\ \pm\left(\alpha, \varpi_{i}\right)>0}}[\alpha]_{i} .
$$

Note that $s_{\sigma, i}^{+}+s_{\sigma, i}^{-}$is larger than half of the codimension of $K^{i} \cdot \sigma \lambda$ in $K \cdot \lambda$.

Theorem 1.4.10 Let $\mathfrak{c}_{+}$and $\mathfrak{c}_{-}$be two adjacent connected component of regular values of $\Phi: K \cdot \lambda \rightarrow \mathfrak{t}^{*}$ separated by an hyperplane orthogonal to a fundamental weight $\varpi_{i}$ : we denote $r_{i}$ the commum value $[\xi]_{i}$ for all $\xi$ in this hyperplane. Let $\mathrm{m}_{\mathfrak{c}_{ \pm}}^{\lambda}: \Lambda^{*} \times \mathbb{Z} \longrightarrow \mathbb{Z}$ be the corresponding periodic polynomials which are supported on the sub-lattice $\Xi_{\lambda}:=\left\{(\mu, k) \mid \mu \in k \lambda+\Lambda_{\mathfrak{R}}^{*}\right\}$.

For all $(\mu, k) \in \Xi_{\lambda}$, we have $\mathrm{m}_{\mathfrak{c}_{+}}^{\lambda}(\mu, k)=\mathrm{m}_{\mathfrak{c}_{-}}^{\lambda}(\mu, k)$ when the integer $[\mu]_{i}-k r_{i}$ satisfies

$$
-s_{i}^{-}<[\mu]_{i}-k r_{i}<s_{i}^{+} .
$$

Here the positive integer $s_{i}^{ \pm}$are defined by

$$
s_{i}^{ \pm}=\inf _{[\sigma] \in \mathcal{A}\left(\mathfrak{c}_{+}, \mathfrak{c}_{-}\right)} s_{\sigma, i}^{ \pm} .
$$

When $\mathcal{A}\left(\mathfrak{c}_{+}, \mathfrak{c}_{-}\right)$is reduced to one element $\sigma$, for example if $\lambda$ is "generic", the integer $s_{i}^{+}+s_{i}^{-}$is larger than half of the codimension of $K^{i} \cdot \sigma \lambda$ in $K \cdot \lambda$.

Another way to express the result of Theorem 1.4.10 is to introduce like in [36] the convex polytope

$$
\square\left(\mathfrak{c}_{+}, \mathfrak{c}_{-}\right)=\bigcap_{\sigma \in \mathcal{A}\left(\mathfrak{c}_{+}, \mathfrak{c}_{-}\right)}\left(\sum_{(\alpha, \sigma \lambda)>0}[0,1[\alpha) .\right.
$$

Let $\Delta$ be the hyperplane which separates $\mathfrak{c}_{+}$and $\mathfrak{c}_{-}$. Equation (1.94) is equivalent to saying that

$$
\mathrm{m}_{\mathfrak{c}_{+}}^{\lambda}(\mu, k)=\mathrm{m}_{\mathfrak{c}_{-}}^{\lambda}(\mu, k) \quad \text { if } \quad \mu \in k \Delta+\square\left(\mathfrak{c}_{+}, \mathfrak{c}_{-}\right) .
$$

Corollary 1.4.11 Let $\mathfrak{c}$ be a connected component of regular values of $\Phi$ which is bording a facet of the polytope $\Phi(K \cdot \lambda)$ orthogonal to the fundamental weight $\varpi_{i}$ : the facet is $\operatorname{conv}\left(W^{i} \cdot \sigma \lambda\right)$ for a unique $\sigma \in W / W^{i}$. We suppose that $\varpi_{i}$ is pointing out of $\mathfrak{c}$. We denote $r_{i}$ the commum value $[\xi]_{i}$ for all $\xi$ in the facet. For all $(\mu, k) \in \Xi_{\lambda}$, we have $\mathrm{m}_{\mathfrak{c}}^{\lambda}(\mu, k)=0$ when the integer $[\mu]_{i}-k r_{i}$ satisfies

$$
-s_{\sigma, i}^{-}<[\mu]_{i}-k r_{i}<s_{\sigma, i}^{+} .
$$


Proof. Theorem 1.4.10 is a direct consequence of Theorem 1.3.24. The main difference between them is the decomposition of the lattice supporting the periodic polynomials. In the former we use the decomposition $\Lambda^{*}=$ $\Lambda_{\mathfrak{t}_{\Delta}}^{*} \oplus \Lambda_{\mathfrak{t} / \mathfrak{t}_{\Delta}}^{*}$ associated to the choice of a subtorus $T / T_{\Delta}$. Here we use the decomposition $\Lambda_{\mathfrak{R}}^{*}=\mathbb{Z} \alpha_{i} \oplus \sum_{k \neq i} \mathbb{Z} \alpha_{k}$. Note first that for $(\mu, k) \in \Xi_{\lambda}$, we have $\mu-\sigma \lambda \in \Lambda_{\mathfrak{R}}^{*}$ and then $[\mu-\sigma \lambda]_{i}=[\mu]_{i}-k r_{i}$ is an integer.

We start like after Proposition 1.3.23: $\mathrm{m}_{\mathfrak{c}_{+}}^{\lambda}(\mu, k)-\mathrm{m}_{\mathfrak{c}_{-}}^{\lambda}(\mu, k)$ is equal to the $\mu$-mutiplicity in $\sum_{\sigma \in \mathcal{A}\left(\mathfrak{c}_{+}, \mathfrak{c}_{-}\right)} A_{\sigma}^{-}-A_{\sigma}^{+}$where

$$
A_{\sigma}^{ \pm}=R R_{0}^{\xi}\left(K^{i} \cdot \sigma \lambda, \mathbb{C}_{[\lambda]}^{\otimes k} \otimes\left[\wedge_{\mathbb{C}}^{\bullet} \overline{\mathcal{N}}_{\sigma, i}\right]_{\mp \varpi_{i}}^{-1}\right)
$$

Here $\xi$ belongs to the relative interior of $\overline{\mathfrak{c}_{+}} \cap \overline{\mathfrak{c}_{+}}$, the line bundle $\mathbb{C}_{[\lambda]}^{\otimes k}$ is equal to $K^{i} \times_{K^{i} \cap K^{\sigma \lambda}} \mathbb{C}_{k \sigma \lambda}$ and $\left[\wedge_{\mathbb{C}}^{\bullet} \overline{\mathcal{N}}_{\sigma, i}\right]_{ \pm \varpi_{i}}^{-1}$ corresponds to $(-1)^{\mathrm{rk}_{\mathbb{C}}\left(N_{\sigma, i}^{ \pm}\right)}$ times

$$
K^{i} \times_{K^{i} \cap K^{\sigma \lambda}}\left(\operatorname{det}\left(N_{\sigma, i}^{ \pm}\right) \otimes S^{\bullet}\left(\left(N_{\sigma, i} \otimes \mathbb{C}\right)^{ \pm}\right)\right),
$$

with

$$
N_{\sigma, i}^{ \pm}=\sum_{\substack{(\alpha, \sigma \lambda)>0 \\ \pm\left(\alpha, \varpi_{i}\right)>0}} \mathfrak{k}_{\alpha}
$$

and

$$
\left(N_{\sigma, i} \otimes \mathbb{C}\right)^{ \pm}=\sum_{\substack{(\alpha, \sigma \lambda) \neq 0 \\ \pm\left(\alpha, \varpi_{i}\right)>0}} \mathfrak{k}_{\alpha}
$$

Now we can apply Remark 1.3 .17 with the subgroup $H \subset T$ equal to the center $Z\left(K^{i}\right)$ of $K^{i}$ : an element $\gamma \in \Lambda^{*}$ belong to $\sum_{k \neq i} \mathbb{Z} \alpha_{k}$ if and only if $t^{\gamma}=1$ for all $t \in Z\left(K^{i}\right)$.

The group $Z\left(K^{i}\right)$ acts trivially on the manifolds $K^{i} \cdot \sigma \lambda$, and with the characters associated to the weights

$$
k \sigma \lambda+\sum_{\substack{(\alpha, \sigma \lambda)>0 \\\left(\alpha, \varpi_{i}\right)>0}} \alpha+\delta \quad \text { with } \quad\left(\delta, \varpi_{i}\right) \geq 0
$$

on the bundle $\mathbb{C}_{[\lambda]}^{\otimes k} \otimes\left[\wedge{ }_{\mathbb{C}} \overline{\mathcal{N}}_{\sigma, i}\right]_{\varpi_{i}}^{-1}$, and with the characters associated to the weights

$$
k \sigma \lambda+\sum_{\substack{(\alpha, \sigma \lambda)>0 \\\left(\alpha, \varpi_{i}\right)<0}} \alpha+\delta \quad \text { with } \quad\left(\delta, \varpi_{i}\right) \leq 0
$$

on the bundle $\mathbb{C}_{[\lambda]}^{\otimes k} \otimes\left[\wedge_{\mathbb{C}}^{\bullet} \overline{\mathcal{N}}_{\sigma, i}\right]_{-\varpi_{i}}^{-1}$. Now the $\mu$-multiplicity in $A_{\sigma}^{ \pm}$is not equal to 0 only if

$$
k \sigma \lambda+\sum_{\substack{(\alpha, \sigma \lambda)>0 \\ \pm\left(\alpha, \varpi_{i}\right)>0}} \alpha+\delta-\mu \in \sum_{k \neq i} \mathbb{Z} \alpha_{k} \quad \text { with } \quad \pm\left(\delta, \varpi_{i}\right) \geq 0 .
$$


Condition (1.100) implies that $[\mu]_{i} \geq k[\sigma \lambda]_{i}+s_{\sigma, i}^{+}$or $[\mu]_{i} \leq k[\sigma \lambda]_{i}-s_{\sigma, i}^{-}$. Finally we have prove that $\mathrm{m}_{\mathfrak{c}_{+}}^{\lambda}(\mu, k)=\mathrm{m}_{\mathfrak{c}_{-}}^{\lambda}(\mu, k)$ if

$$
-s_{\sigma, i}^{-}<[\mu]_{i}-k[\sigma \lambda]_{i}<s_{\sigma, i}^{+}
$$

for all $\sigma \in \mathcal{A}\left(\mathfrak{c}_{+}, \mathfrak{c}_{-}\right)$.

\subsubsection{The case of $\mathrm{SU}(n)$}

Let $T$ be the maximal torus of $\mathrm{SU}(n)$ consisting of the diagonal matrices. The dual $\mathfrak{t}^{*}$ can be identified with the subspace $x_{1}+\cdots+x_{n}=0$ of $\mathbb{R}^{n}$. The roots are $\mathfrak{R}=\left\{e_{i}-e_{j} \mid 1 \leq i \neq j \leq n\right\}$ and we will choose the positives ones to be $\mathfrak{R}^{+}=\left\{e_{i}-e_{j} \mid 1 \leq i<j \leq n\right\}$. The simple roots are then $\alpha_{i}=e_{i}-e_{i+1}$, for $1 \leq i \leq n-1$, and for these simple roots, the fundamental weights are

$$
\varpi_{k}=\frac{1}{n}(\underbrace{n-k, n-k, \cdots, n-k}_{k \text { times }}, \underbrace{-k,-k, \cdots,-k}_{n-k \text { times }}), \quad 1 \leq k \leq n-1 .
$$

Consider now the coadjoint orbit $O_{\lambda}$ for $\lambda \in \mathfrak{t}^{*}$. Let $\Phi: O_{\lambda} \rightarrow \mathfrak{t}^{*}$ the moment map associated to the Hamiltonian action of $T$ on $O_{\lambda}$. The center of $\mathrm{SU}(n)$, that we denote $\mathbb{Z}_{n}$ corresponds to the set of matrices $z I$ with $z^{n}=1$. Recall the following well-known fact.

Lemma 1.4.12 Let $\xi$ be a regular value of $\Phi: O_{\lambda} \rightarrow \mathfrak{t}^{*}$. Then for every $m \in \Phi^{-1}(\xi)$ the stabilizer subgroup $T_{m}:=\{t \in T \mid t \cdot m=m\}$ is equal to $\mathbb{Z}_{n}$.

Proof. Since $\xi$ is a regular value, we know that $T_{m}$ is finite for every $m \in \Phi^{-1}(\xi)$. The dual of the Lie algebra $\mathfrak{s u}(n)$ decomposes as $\mathfrak{s u}(n)^{*}=\mathfrak{t}^{*} \oplus$ $\sum_{\alpha \in \mathfrak{R}^{+}} \mathfrak{s u}(n)_{\alpha}^{*}$ where $\mathfrak{s u}(n)_{\alpha}^{*} \simeq \mathbb{C}_{-\alpha}$ as $T$-module. For $m \in \Phi^{-1}(\xi)$, we have $m=m_{0}+\sum_{\alpha \in \mathfrak{R}^{+}} m_{\alpha}$ with $m_{\alpha} \in \mathfrak{s u}(n)_{\alpha}^{*}$, and then $T_{m}=\cap_{m_{\alpha} \neq 0} \operatorname{ker}\left(t \mapsto t^{\alpha}\right)$. So the lattice $\Lambda_{m}^{*}$ generated by the set $\left\{\alpha \in \mathfrak{R}^{+} \mid m_{\alpha} \neq 0\right\}$ is a subgroup of $\Lambda_{\mathfrak{R}}^{*}$ with $\Lambda_{\mathfrak{R}}^{*} / \Lambda_{m}^{*}$ finite. We have to show that $\Lambda_{m}^{*}=\Lambda_{\mathfrak{R}}^{*}$. For this purpose we introduce the following equivalence relation on $\{1, \ldots, n\}$ :

$$
i \sim j \Longleftrightarrow e_{i}-e_{j} \in \Lambda_{m}^{*} .
$$

Suppose that $\{1, \ldots, n\} / \sim$ is not reduced to a point: let $C_{1}$ and $C_{2}$ be two distinct equivalent classes and let $\beta=\left(\beta_{1}, \ldots, \beta_{n}\right)$ be the element of $\mathfrak{t}^{*}$ defined by: $\beta_{i}=\frac{1}{\left|C_{1}\right|}$ if $i \in C_{1}, \beta_{i}=\frac{-1}{\left|C_{2}\right|}$ if $i \in C_{2}$, and $\beta_{i}=0$ in the other cases. We see then that $(\beta, \alpha)=0$ for all $\alpha \in \Lambda_{m}^{*}$ : it is in contradiction with the fact that $\Lambda_{\mathfrak{R}}^{*} / \Lambda_{m}^{*}$ is finite. We have proved that $e_{i}-e_{j} \in \Lambda_{m}^{*}$ for all $i, j \in\{1, \ldots, n\}$.

We are in the particularly nice situation where the symplectic reduction $\left(O_{\lambda}\right)_{\xi}=\Phi^{-1}(\xi) / T$ is a smooth manifold for any regular value $\xi$.

Suppose now that $\lambda$ is a positive weight, and let $\mathfrak{c}$ a connected component of regular values of $\Phi: O_{\lambda} \rightarrow \mathfrak{t}^{*}$. We know that $\mathrm{m}_{\mathfrak{c}}^{\lambda}: \Lambda^{*} \times \mathbb{Z} \longrightarrow \mathbb{Z}$ is supported on the sub-lattice $\Xi_{\lambda}:=\left\{(\mu, k) \mid \mu \in k \lambda+\Lambda_{\mathfrak{R}}^{*}\right\}$. 
Corollary 1.4.13 The map $\mathrm{m}_{\mathfrak{c}}^{\lambda}: \Xi_{\lambda} \longrightarrow \mathbb{Z}$ is a polynomial of degree $\frac{(n-1)(n-2)}{2}-d_{\lambda}$, where $d_{\lambda}$ is the number of positive roots orthogonal to $\lambda$. .

Proof. Take $\xi \in \mathfrak{c}$. Following Proposition 1.3.18, the periodic-polynomial $\mathrm{m}_{\mathfrak{c}}^{\lambda}$ is defined by $\mathrm{m}_{\mathfrak{c}}^{\lambda}(\mu, k)=R R\left(\left(O_{\lambda}\right)_{\xi}, \mathcal{L}_{\xi, \mu}^{k}\right)$ for all $(\mu, k) \in \Xi_{\lambda}$. Here $\left(O_{\lambda}\right)_{\xi}=$ $\Phi^{-1}(\xi) / T$ is a smooth manifold, and the line bundle $\mathcal{L}_{\xi, \mu}^{k}=\left(\left.L^{\otimes k}\right|_{\Phi^{-1}(\xi)} \otimes\right.$ $\left.\mathbb{C}_{-\mu}\right) / T$ is also smooth since the center $\mathbb{Z}_{n}$ acts trivially on $\left.L^{\otimes k}\right|_{\Phi^{-1}(\xi)} \otimes$ $\mathbb{C}_{-\mu}$. Now the Atiyah-Singer integral formula for the Riemann-Roch number $R R\left(\left(O_{\lambda}\right)_{\xi}, \mathcal{L}_{\xi, \mu}^{k}\right)$ shows that $\mathrm{m}_{\mathfrak{c}}^{\lambda}$ is a polynomial of degree $\frac{\operatorname{dim}\left(O_{\lambda}\right)_{\xi}}{2}=\frac{\operatorname{dim} O_{\lambda}}{2}-$ $(n-1)=\frac{(n-1)(n-2)}{2}-d_{\lambda}$.

Now we rewrite Theorem 1.4.10 for the group $\operatorname{SU}(n)$. Let $\lambda=\left(\lambda_{1} \geq\right.$ $\cdots \geq \lambda_{n}$ ) be a positive weight and let $\mathfrak{c}_{+}$and $\mathfrak{c}_{-}$be two adjacent connected components of regular values of $\Phi: O_{\lambda} \rightarrow \mathfrak{t}^{*}$ separated by an hyperplane orthogonal to a fundamental weight $\varpi_{i}$ : the vector $\varpi_{i}$ is pointing out of $\mathfrak{c}_{-}$, and let $\left(\varpi_{i}, \xi\right)-r_{i}=0$ be the equation of this hyperplane. We consider the linear map

$$
Q(\xi, t):=\left(\varpi_{i}, \xi\right)-t r_{i} .
$$

The hyperplane $\{Q=0\} \subset \mathfrak{t}^{*} \times \mathbb{R}$ separates Cone $\left(\mathfrak{c}_{+}\right)$and Cone $\left(\mathfrak{c}_{-}\right)$.

The conditions $\left(e_{k}-e_{l}, \sigma \lambda\right)>0$ and $\left(e_{k}-e_{l}, \varpi_{i}\right)>0$ are respectively equivalent to $\lambda_{\sigma(k)}>\lambda_{\sigma(l)}$ and $k \leq i<l$. For $\mathrm{SU}(n)$, the number $[\alpha]_{i}$ is equal to 0,1 or -1 for any roots $\alpha$ and any $i=1, \cdots, n-1$. Hence for every $\sigma \in \mathcal{A}\left(\mathfrak{c}_{+}, \mathfrak{c}_{-}\right)$, the integers $s_{\sigma, i}^{-}, s_{\sigma, i}^{+} \geq 0$ introduced in Definition (1.4.9) are equal to

$$
\begin{array}{lll}
s_{\sigma, i}^{+}=\operatorname{rk}_{\mathbb{C}}\left(N_{\sigma, i}^{+}\right)=\sharp\{k \leq i<l \quad \text { such that } & \left.\lambda_{\sigma(k)}>\lambda_{\sigma(l)}\right\}, \\
s_{\sigma, i}^{-}=\operatorname{rk}_{\mathbb{C}}\left(N_{\sigma, i}^{-}\right)=\sharp\{k \leq i<l \quad \text { such that } & \left.\lambda_{\sigma(k)}<\lambda_{\sigma(l)}\right\},
\end{array}
$$

and the sum $s_{\sigma, i}^{+}+s_{\sigma, i}^{-}$is equal to half of the codimension of $K^{i} \cdot \sigma \lambda$ in $K \cdot \lambda$, that is $s_{\sigma, i}^{+}+s_{\sigma, i}^{-}=i(n-i)-\operatorname{dim}\left(K^{\sigma \lambda} / K^{i} \cap K^{\sigma \lambda}\right) / 2$.

Now we precise the results of [10].

Theorem 1.4.14 $\bullet$ The polynomial $\mathrm{m}_{\mathfrak{c}_{-}}^{\lambda}-\mathrm{m}_{\mathfrak{c}_{+}}^{\lambda}: \Xi_{\lambda} \rightarrow \mathbb{Z}$ is divible by the linear factors

$$
\left(Q-s_{i}^{-}+1\right),\left(Q-s_{i}^{-}+2\right), \ldots, Q, \ldots,\left(Q+s_{i}^{+}-2\right),\left(Q+s_{i}^{+}-1\right),
$$

where $s_{i}^{ \pm}=\inf _{[\sigma] \in \mathcal{A}\left(\mathfrak{c}_{+}, \mathfrak{c}_{-}\right)} s_{\sigma, i}^{ \pm}$.

- The linear factors $\left(Q-s_{i}^{-}\right)$and $\left(Q-s_{i}^{+}\right)$do not divide $\mathrm{m}_{\mathbf{c}_{-}}^{\lambda}-\mathrm{m}_{\mathbf{c}_{+}}^{\lambda}$.

Proof. The first part is the translation of Theorem 1.4.10. We have just to prove that the linear factors $\left(Q-s_{i}^{-}\right)$and $\left(Q-s_{i}^{+}\right)$do not divide $\mathrm{m}_{\mathfrak{c}_{-}}^{\lambda}-\mathrm{m}_{\mathfrak{c}_{+}}^{\lambda}$. This point is a direct application of Proposition 1.3.25. The only fact we use here is that $\operatorname{rk}_{\mathbb{C}}\left(N_{\sigma, i}^{ \pm}\right)=s_{\sigma, i}^{ \pm}$. So the number $\operatorname{rk}_{\mathbb{C}}\left(N_{\sigma, i}^{ \pm}\right)$is constant for all $\sigma \in \mathcal{A}\left(\mathfrak{c}_{+}, \mathfrak{c}_{-}\right)$for which $s_{\sigma, i}^{ \pm}=s_{i}^{ \pm}$. 
We rewrite now Theorem 1.4.14 in the particular case where $\mathcal{A}\left(\mathfrak{c}_{+}, \mathfrak{c}_{-}\right)$ contains just one element: it happens when $\lambda$ is a "generic" positive weight (see Definition 1.4.5), or when $\mathfrak{c}_{+}$does not intersect $\Phi\left(O_{\lambda}\right)$. Here a positive weight $\lambda=\left(\lambda_{1} \geq \cdots \geq \lambda_{n}\right)$ is "generic" if for every couple of permutations $\sigma, \sigma^{\prime}$ and any $k=1, \cdots, n-1$, we have

$$
\sum_{i=1}^{k} \lambda_{\sigma(i)} \neq \sum_{i=1}^{k} \lambda_{\sigma^{\prime}(i)}
$$

when $\left(\lambda_{\sigma(1)}, \cdots, \lambda_{\sigma(n)}\right) \notin \mathfrak{S}_{k} \times \mathfrak{S}_{n-k}\left(\lambda_{\sigma^{\prime}(1)}, \cdots, \lambda_{\sigma^{\prime}(n)}\right)$.

Corollary 1.4.15 Let $\lambda$ be a regular weight. Let $\mathfrak{c}_{+}$and $\mathfrak{c}_{-}$be two adjacent connected components of regular values of $\Phi: O_{\lambda} \rightarrow \mathfrak{t}^{*}$ and suppose that $\mathcal{A}\left(\mathfrak{c}_{+}, \mathfrak{c}_{-}\right)$contains just one element $\sigma$. Then the polynomial $\mathrm{m}_{\mathfrak{c}_{-}}^{\lambda}-\mathrm{m}_{\mathfrak{c}_{+}}^{\lambda}$ : $\Xi_{\lambda} \rightarrow \mathbb{Z}$ is divible by the $i(n-i)$ linear factors

$$
\left(Q-s_{i}^{-}+1\right),\left(Q-s_{i}^{-}+2\right), \ldots, q, \ldots,\left(Q+s_{i}^{+}-2\right),\left(Q+s_{i}^{+}-1\right),
$$

where $s_{i}^{ \pm}=s_{\sigma, i}^{ \pm}$are defined by (1.102) and (1.103). Moreover the linear factors $\left(Q-s_{i}^{-}\right)$and $\left(Q-s_{i}^{+}\right)$do not divide $\mathrm{m}_{\mathfrak{c}_{-}}^{\lambda}-\mathrm{m}_{\mathfrak{c}_{+}}^{\lambda}$.

\subsection{Vector partition functions}

Let $T$ be a torus with Lie algebra $\mathfrak{t}$ and let $\Lambda^{*} \subset \mathfrak{t}^{*}$ be the weight lattice. Let $R=\left\{\alpha_{1}, \ldots, \alpha_{d}\right\}$ be a subset of not necessarily distinct elements of $\Lambda^{*}$ which are in an open halfspace of $t^{*}$. We associate with the collection $R$ a function

$$
N_{R}: \Lambda^{*} \longrightarrow \mathbb{N}
$$

called the vector partition function associated to $R$. By definition, for a weight $\mu$, the value $N_{R}(\mu)$ is the number of solutions of the equation

$$
\sum_{j=1}^{d} k_{j} \alpha_{j}=\mu, \quad k_{j} \in \mathbb{Z}^{\geq 0}, \quad j=1, \ldots, d .
$$

Let $C(R) \subset \mathfrak{t}^{*}$ be the closed convex cone generated by the elements of $R$, and denote by $\Lambda_{R}^{*} \subset \Lambda^{*}$ the sublattice generated by $R$. Obviously, $N_{R}(\mu)$ vanishes if $\mu$ does not belong to $C(R) \cap \Lambda_{R}^{*}$.

Suppose now that $R$ generates the vector space $\mathfrak{t}^{*}$. Following [36], we will call a vector singular with respect to $R$ if it is in a cone $C(\nu)$ generated by a subset $\nu \subset R$ of cardinality strictly less than $\operatorname{dim} T$. The connected components of $\mathrm{t}^{*} \backslash\{$ singular vectors $\}$ are called conic chambers. The periodic polynomial behavior of $N_{R}$ on closures of conic chambers of the cone $C(R)$ is proved in [35]. We have the following refinement due to Szenes and Vergne [36]. Let us introduce the convex polytope 


$$
\square(R)=\sum_{j=1}^{d}[0,1] \alpha_{j}
$$

We remark that $\mathfrak{c}-\square(R)$ is a neighborhood of $\overline{\mathfrak{c}}$ for any conic chamber $\mathfrak{c}$ of the cone $C(R)$. We have the following qualitative result.

Theorem 1.5.1 ([36]) Let $\mathfrak{c}$ be a conic chamber of the cone $C(R)$. There exists a periodic polynomial $P_{\mathfrak{c}}$ on $\Lambda^{*}$ such that for each $\mu \in \mathfrak{c}-\square(R)$, we have

$$
N_{R}(\mu)=P_{\mathfrak{c}}(\mu) \text {. }
$$

In Section 1.5.4 we will give another proof of Theorem 1.5.1.

Let $\mathfrak{c}_{ \pm} \subset \mathfrak{t}^{*}$ be two adjacent conic chambers separated by the hyperplane $\Delta=\left\{\xi \in \mathfrak{t}^{*} \mid\langle\xi, \beta\rangle=0\right\}$. Here $\beta \in \mathfrak{t}$ is chosen so that $\mathfrak{c}_{ \pm} \subset\left\{\xi \in \mathfrak{t}^{*} \mid \pm\langle\xi, \beta\rangle>\right.$ $0\}$. The aim of this Section is to give a wall-crossing formula for the periodic polynomial $P_{\mathfrak{c}_{+}}-P_{\mathfrak{c}_{-}}$.

Note that the vector space $\Delta$ is generated by $R \cap \Delta$. We polarize the elements of $R$ that are outside $\Delta$. We define

$$
\begin{gathered}
R^{\prime}=\left\{\epsilon_{j} \alpha_{j} \mid\left\langle\alpha_{j}, \beta\right\rangle \neq 0 \text { and } \epsilon_{j}=\operatorname{sign}\left\langle\alpha_{j}, \beta\right\rangle\right\}, \\
\delta^{ \pm}=\sum_{ \pm\left\langle\alpha_{j}, \beta\right\rangle>0} \alpha_{j},
\end{gathered}
$$

and

$$
r^{ \pm}=\sharp\left\{j \mid \pm\left\langle\alpha_{j}, \beta\right\rangle>0\right\} .
$$

We now look at the vector space $\Delta$ equipped with the subset $R \cap \Delta \subset$ $\Lambda^{*} \cap \Delta$ which lie enterely in an open halfspace: let $N_{R \cap \Delta}: \Lambda^{*} \cap \Delta \rightarrow \mathbb{N}$ be the corresponding vector partition function. It is easy to see that $\overline{\mathfrak{c}_{+}} \cap \overline{\bar{c}_{-}}$is contained in the closure of a conic chamber $\mathfrak{c}^{\prime} \subset \Delta$ relative to $R \cap \Delta$. Following Proposition 1.5.1 there exists a periodic polynomial $P_{\mathfrak{c}^{\prime}}$ on $\Lambda^{*} \cap \Delta$ such that for each $\mu \in \overline{\mathfrak{c}^{\prime}} \cap \Lambda^{*}$, we have

$$
N_{R \cap \Delta}(\gamma)=P_{\mathfrak{c}^{\prime}}(\gamma)
$$

Let $N_{R^{\prime}}: \Lambda^{*} \rightarrow \mathbb{N}$ be the vector partition function associated to the polarized set of weight $R^{\prime}$ (see (1.106)). The main result of this Section is the following

Theorem 1.5.2 The periodic polynomial $P_{\mathfrak{c}_{+}}-P_{\mathfrak{c}_{-}}: \Lambda^{*} \rightarrow \mathbb{Z}$ satisfies

$$
P_{\mathfrak{c}_{+}}(\mu)-P_{\mathfrak{c}_{-}}(\mu)=\sum_{\gamma \in \Lambda^{*} \cap \Delta} D(\mu-\gamma) P_{\mathfrak{c}^{\prime}}(\gamma), \quad \mu \in \Lambda^{*},
$$

where $D: \Lambda^{*} \rightarrow \mathbb{Z}$ is defined by

$$
D(\mu)=(-1)^{r^{-}} N_{R^{\prime}}\left(\mu+\delta^{-}\right)-(-1)^{r^{+}} N_{R^{\prime}}\left(-\mu-\delta^{+}\right) .
$$


The proof of Theorem 1.5.2 will be given in Section 1.5.5.

Corollary 1.5.3 $P_{\mathfrak{c}_{+}}(\mu)=P_{\mathfrak{c}_{-}}(\mu)$ for all the weights $\mu \in \Lambda^{*}$ satisfying the condition

$$
-\left\langle\delta^{+}, \beta\right\rangle<\langle\mu, \beta\rangle<-\left\langle\delta^{-}, \beta\right\rangle .
$$

The former ineqalities are optimal since

$$
\left(P_{\mathfrak{c}_{+}}-P_{\mathfrak{c}_{-}}\right)\left(-\delta^{-}+\gamma\right)=(-1)^{r^{-}} P_{\mathfrak{c}^{\prime}}(\gamma)
$$

and

$$
\left(P_{\mathfrak{c}_{+}}-P_{\mathfrak{c}_{-}}\right)\left(-\delta^{+}+\gamma\right)=(-1)^{1+r^{+}} P_{\mathfrak{c}^{\prime}}(\gamma)
$$

for all $\gamma \in \Lambda^{*} \cap \Delta$.

Proof. In (1.109), the term $D(\mu-\gamma) P_{\mathfrak{c}^{\prime}}(\gamma)$ does not vanish only if $\mu-\gamma \in$ $-\delta^{-}+C\left(R^{\prime}\right)$ or $-(\mu-\gamma) \in \delta^{+}+C\left(R^{\prime}\right)$ for some $\gamma \in C(R \cap \Delta)$. These two conditions impose respectively that $\langle\mu, \beta\rangle \geq-\left\langle\delta^{-}, \beta\right\rangle$ and $\langle\mu, \beta\rangle \leq-\left\langle\delta^{+}, \beta\right\rangle$. If one take $\mu=-\delta^{-}+\gamma$ with $\gamma \in \Lambda^{*} \cap \Delta,(1.109)$ becomes $\left(P_{\mathfrak{c}_{+}}-P_{\mathfrak{c}_{-}}\right)\left(-\delta^{-}+\right.$ $\gamma)=\sum_{\gamma^{\prime} \in \Lambda^{*} \cap \Delta} D\left(-\delta^{-}+\gamma-\gamma^{\prime}\right) P_{\mathfrak{c}^{\prime}}\left(\gamma^{\prime}\right)$ with

$$
D\left(-\delta^{-}+\gamma-\gamma^{\prime}\right)=(-1)^{r^{-}} N_{R^{\prime}}\left(\gamma-\gamma^{\prime}\right)-(-1)^{r^{+}} N_{R^{\prime}}\left(\delta^{-}-\delta^{+}-\gamma+\gamma^{\prime}\right)
$$

Since the cone $C\left(R^{\prime}\right)$ intersects $\Delta$ only at $\{0\}, N_{R^{\prime}}\left(\gamma-\gamma^{\prime}\right)=0$ if $\gamma \neq \gamma^{\prime}$. Since $\left\langle\delta^{-}-\delta^{+}, \beta\right\rangle<0$ we always have $N_{R^{\prime}}\left(\delta^{-}-\delta^{+}-\gamma+\gamma^{\prime}\right)=0$. We get finally that $\left(P_{\mathfrak{c}_{+}}-P_{\mathfrak{c}_{-}}\right)\left(-\delta^{-}+\gamma\right)=(-1)^{r^{-}} P_{\mathfrak{c}^{\prime}}(\gamma)$. One can show in the same way that $\left(P_{\mathfrak{c}_{+}}-P_{\mathfrak{c}_{-}}\right)\left(-\delta^{+}+\gamma\right)=-(-1)^{r^{+}} P_{\mathfrak{c}^{\prime}}(\gamma)$.

\subsubsection{Quantization of $\mathbb{C}^{d}$}

We consider the complex vector space $\mathbb{C}^{d}$ equipped with the canonical symplectic form $\Omega=\frac{i}{2} \sum_{i=1}^{d} d z_{j} \wedge d \bar{z}_{j}$. The standard complex struture $J$ on $\mathbb{C}^{d}$ is compatible with $\Omega$. Let $T$ be a torus, let $\alpha_{j} \in \mathfrak{t}^{*}, j=1, \ldots, d$ be weights of $T$, and let $T$ acts on $\mathbb{C}^{d}$ as

$$
t \cdot\left(z_{1}, \ldots, z_{d}\right)=\left(t^{-\alpha_{1}} z_{1}, \ldots, t^{-\alpha_{d}} z_{d}\right)
$$

The action of $T$ preserve the symplectic form $\Omega$ and the moment map associated with this action is

$$
\Phi(z)=\frac{1}{2} \sum_{i=1}^{d}\left|z_{j}\right|^{2} \alpha_{j} .
$$

The pre-quantization data $(L,\langle\rangle,, \nabla)$ on the Hamiltonian $T$-manifold $\left(\mathbb{C}^{d}, \Omega, \Phi\right)$ is a trivial line bundle $L$ with a trivial action of $T$ equipped with the Hermitian structure $\left\langle s, s^{\prime}\right\rangle_{z}=e^{\frac{-|z|^{2}}{2}} s \overline{s^{\prime}}$ and the Hermitian connexion $\nabla=d-\theta$ where $\theta=\frac{1}{2} \sum_{i=1}^{d} \bar{z}_{j} d z_{j}$. 
The quantization of the Hamiltonian $T$-manifold $\left(\mathbb{C}^{d}, \Omega\right)$, that we denote $\mathcal{Q}^{T}\left(\mathbb{C}^{d}\right)$, is the Bargman space of entire holomorphic functions on $\mathbb{C}^{d}$ which are $\mathcal{L}^{2}$ integrable with respect to the Gaussian measure $e^{\frac{-|z|^{2}}{2}} \Omega^{d}$.

We suppose now that the set of weights $R=\left\{\alpha_{1}, \ldots, \alpha_{d}\right\}$ is polarized by $\eta \in \mathfrak{t}$, which means that $\left\langle\alpha_{j}, \eta\right\rangle>0$ for all $j$. The $T$-representation $\mathcal{Q}^{T}\left(\mathbb{C}^{d}\right)$ is then admissible and we have the following equality in $R^{-\infty}(T)$ :

$$
\mathcal{Q}^{T}\left(\mathbb{C}^{d}\right)=\sum_{\mu \in \Lambda^{*}} N_{R}(\mu) \mathbb{C}_{\mu}
$$

where $N_{R}: \Lambda^{*} \rightarrow \mathbb{N}$ is the vector partition function associated to $R$. In other words, the generalized character of $\mathcal{Q}^{T}\left(\mathbb{C}^{d}\right)$ coincides with the generalized character of the symmetric algebra $S^{\bullet}\left(\overline{\mathbb{C}^{d}}\right)$, where $\overline{\mathbb{C}^{d}}$ means $\mathbb{C}^{d}$ with the opposite complex structure.

For the remaining part of Section 1.5, we assume that the set of weights $R=\left\{\alpha_{1}, \ldots, \alpha_{d}\right\}$ is polarized, and generates the vector space $\mathbf{t}^{*}$. The first assumption is equivalent to the fact that the moment map $\Phi: \mathbb{C}^{d} \rightarrow \mathfrak{t}^{*}$ is proper, and the second assumption is equivalent to the fact that the generic stabiliser of $T$ on $\mathbb{C}^{d}$ is finite. Notice that the vectors of $\mathfrak{t}^{*}$ which are singular with respect to $R$ correspond to the singular values of $\Phi$.

In the next section we will show that $\mathcal{Q}^{T}\left(\mathbb{C}^{d}\right)$, viewed as an element of $R^{-\infty}(T)$, can be realized as the index of transversally elliptic symbols on $\mathbb{C}^{d}$. After we will apply the techniques developped in Section 1.3. The main difference here is that we work with the non-compact manifold $\mathbb{C}^{d}$.

\subsubsection{Transversally elliptic symbols on $\mathbb{C}^{d}$}

Let $p: \mathbf{T} \mathbb{C}^{d} \rightarrow \mathbb{C}^{d}$ be the canonical projection. We consider the Thom symbol

$$
\operatorname{Thom}\left(\mathbb{C}^{d}\right) \in \Gamma\left(\mathbf{T} \mathbb{C}^{d}, \operatorname{hom}\left(p^{*}\left(\wedge_{\mathbb{C}}^{\text {even }} \mathbf{T} \mathbb{C}^{d}\right), p^{*}\left(\wedge_{\mathbb{C}}^{\text {odd }} \mathbf{T} \mathbb{C}^{d}\right)\right)\right)
$$

associated to the standard Hermitian structure on $\mathbb{C}^{d}$. Obviously the symbol Thom $\left(\mathbb{C}^{d}\right)$ is not elliptic since its characteristic set is equal to the zero section in $\mathbf{T} \mathbb{C}^{d}$ (hence is not compact).

Now we deform the symbol $\operatorname{Thom}\left(\mathbb{C}^{d}\right)$ in order to obtain transversally elliptic symbols. Since $\mathbb{C}^{d}$ can be realized as an open subset of a compact $T$-manifold we have a well defined index map

$$
\operatorname{Index}_{\mathbb{C}^{d}}^{T}: \mathbf{K}_{T}\left(\mathbf{T}_{T} \mathbb{C}^{d}\right) \longrightarrow R^{-\infty}(T) .
$$

Definition 1.5.4 For any $\eta \in \mathfrak{t}$, we define the symbol $\mathrm{Thom}^{\eta}\left(\mathbb{C}^{d}\right)$ by

$$
\operatorname{Thom}^{\eta}\left(\mathbb{C}^{d}\right)(z, v)=\operatorname{Thom}\left(\mathbb{C}^{d}\right)\left(z, v-\eta_{\mathbb{C}^{d}}(z)\right), \quad(z, v) \in \mathbf{T} \mathbb{C}^{d},
$$

where $\eta_{\mathbb{C}^{d}}$ is the vector field on $\mathbb{C}^{d}$ generated by $\eta$. 
The symbols $\operatorname{Thom}^{\eta}\left(\mathbb{C}^{d}\right)$ were studied in [32]. It is easy to see that $\operatorname{Thom}^{\eta}\left(\mathbb{C}^{d}\right)$ is tranversally elliptic if and only if the vector subspace $\left(\mathbb{C}^{d}\right)^{\eta}$ is reduced to $\{0\}$, i.e. if $\left\langle\alpha_{j}, \eta\right\rangle \neq 0$ for all $j=1, \ldots, d$. We prove in Proposition 5.4. of [32] that

$$
\operatorname{Index}_{\mathbb{C}^{d}}^{T}\left(\operatorname{Thom}^{\eta}\left(\mathbb{C}^{d}\right)\right)=S^{\bullet}\left(\overline{\mathbb{C}^{d}}\right) \quad \text { in } \quad R^{-\infty}(T),
$$

when $\left\langle\alpha_{j}, \eta\right\rangle>0$ for all $j=1, \ldots, d$.

In order to compute the multiplicities $N_{R}(\mu)$ of $\mathcal{Q}^{T}\left(\mathbb{C}^{d}\right)$ we introduce the following tranversally elliptic symbols. Take a scalar product $b(\cdot, \cdot)$ on $\mathfrak{t}^{*}$, and denote by $\xi \mapsto \xi^{b}, \mathfrak{t}^{*} \simeq \mathfrak{t}$ the induced isomorphism. For each $\xi \in \mathfrak{t}^{*}$, the Hamiltonian vector field of the function $\frac{-1}{2}\|\Phi-\xi\|_{b}^{2}$ is the vector field

$$
z \mapsto\left((\Phi(z)-\xi)^{b}\right)_{\mathbb{C}^{d}}(z) .
$$

that we denote $\mathcal{H}^{b}-\xi_{\mathbb{C}^{d}}^{b}$.

Definition 1.5.5 For any $\xi \in \mathfrak{t}^{*}$, and any scalar product $b(\cdot, \cdot)$ on $\mathfrak{t}^{*}$, we define the symbol $\mathrm{Thom}_{\xi, b}\left(\mathbb{C}^{d}\right)$ by

$\operatorname{Thom}_{\xi, b}\left(\mathbb{C}^{d}\right)(z, v)=\operatorname{Thom}\left(\mathbb{C}^{d}\right)\left(z, v-\left(\mathcal{H}^{b}-\xi_{\mathbb{C}^{d}}^{b}\right)(z)\right), \quad(z, v) \in \mathbf{T} \mathbb{C}^{d}$.

Let $\operatorname{Char}\left(\operatorname{Thom}_{\xi, b}\left(\mathbb{C}^{d}\right)\right) \subset \mathbf{T} \mathbb{C}^{d}$ be the characterictic set of $\operatorname{Thom}_{\xi, b}\left(\mathbb{C}^{d}\right)$. We know that Char $\left(\operatorname{Thom}_{\xi, b}\left(\mathbb{C}^{d}\right)\right) \cap \mathbf{T}_{T} \mathbb{C}^{d}$ is equal to the critical set $\operatorname{Cr}(\| \Phi-$ $\xi \|_{b}^{2}$ ) of the function $\|\Phi-\xi\|_{b}^{2}: \mathbb{C}^{d} \rightarrow \mathbb{R}$ (see Section 1.3.2). A straightforward computation gives that $z \in \operatorname{Cr}\left(\|\Phi-\xi\|_{b}^{2}\right)$ if and only if

$$
b\left(\Phi(z)-\xi, \alpha_{j}\right) z_{j}=0 \text { for all } j=1, \ldots, d .
$$

The former relations implies in particular that $b(\Phi(z)-\xi, \Phi(z))=\frac{1}{2} \sum_{j} b(\Phi(z)-$ $\left.\xi, \alpha_{j}\right)\left|z_{j}\right|^{2}=0$. Hence $\|\Phi(z)\|_{b}^{2}=b(\Phi(z), \xi)$ which implies

$$
\|\Phi(z)\|_{b} \leq\|\xi\|_{b} .
$$

Take now $\eta \in \mathfrak{t}$ such that $\left\langle\alpha_{j}, \eta\right\rangle>0$ for all $j$, and let $\eta_{b} \in \mathfrak{t}^{*}$ such that $\left(\eta_{b}\right)^{b}=\eta$. We have then

$$
C_{\eta}\|z\|^{2} \leq\langle\Phi(z), \eta\rangle=b\left(\Phi(z), \eta_{b}\right) \leq\|\Phi(z)\|_{b}\left\|\eta_{b}\right\|_{b}
$$

where $C_{\eta}=\frac{1}{2} \inf _{j}\left\langle\alpha_{j}, \eta\right\rangle$, and $z \mapsto\|z\|^{2}$ is the usual hermitian form on $\mathbb{C}^{d}$. With (1.114) and (1.116) we get the following

Lemma 1.5.6 The critical set $\operatorname{Cr}\left(\|\Phi-\xi\|_{b}^{2}\right) \subset \mathbb{C}^{d}$ is contained in the ball of radius

$$
\frac{\|\xi\|_{b}\left\|\eta_{b}\right\|_{b}}{C_{\eta}}
$$

where $\eta \in \mathfrak{t}$ is such that $C_{\eta}=\frac{1}{2} \inf _{j}\left\langle\alpha_{j}, \eta\right\rangle>0$. 
We have then proved that the symbols $\operatorname{Thom}_{\xi, b}\left(\mathbb{C}^{d}\right)$ are transversally elliptic.

Proposition 1.5.7 The class of the transversally elliptic symbol $\mathrm{Thom}_{\xi, b}\left(\mathbb{C}^{d}\right)$ in $\mathbf{K}_{T}\left(\mathbf{T}_{T} \mathbb{C}^{d}\right)$ does not depend of the data $\xi, b$, and is equal to the class defined by $\operatorname{Thom}^{\eta}\left(\mathbb{C}^{d}\right)$ where $\eta \in \mathfrak{t}$ is chosen so that $\left\langle\alpha_{j}, \eta\right\rangle>0$ for all $j$.

Proof. After Lemma 1.5.6, we know that for any scalar product $b(\cdot, \cdot)$ on $\mathfrak{t}^{*}$, the characteristic set of $\operatorname{Thom}_{0, b}\left(\mathbb{C}^{d}\right)$ intersects $\mathbf{T}_{T} \mathbb{C}^{d}$ at $\{0\}$. If $b_{0}$ and $b_{1}$ are two scalar products on $\mathfrak{t}^{*}$ we consider the family $b_{t}=t b_{1}+(1-$ $t) b_{0}, 0 \leq t \leq 1$, of scalar products on $\mathfrak{t}^{*}$. Hence $\operatorname{Thom}_{0, b_{t}}\left(\mathbb{C}^{d}\right), t \in[0,1]$, defines an homotopy of transversally elliptic symbols. We have proved that $\operatorname{Thom}_{0, b_{0}}\left(\mathbb{C}^{d}\right)=\operatorname{Thom}_{0, b_{1}}\left(\mathbb{C}^{d}\right)$ in $\mathbf{K}_{T}\left(\mathbf{T}_{T} \mathbb{C}^{d}\right)$ for any $\xi \in \mathfrak{t}^{*}$.

Fix now the scalar product $b$ and an element $\xi \in \mathfrak{t}^{*}$. For any $t \in[0,1]$ the characteristic set of $\operatorname{Thom}_{t \xi, b}\left(\mathbb{C}^{d}\right)$ intersects $\mathbf{T}_{T} \mathbb{C}^{d}$ in the ball of radius

$$
\frac{\|\xi\|_{b}\left\|\eta_{b}\right\|_{b}}{C_{\eta}}
$$

Hence $\operatorname{Thom}_{t \xi, b}\left(\mathbb{C}^{d}\right), t \in[0,1]$, defines an homotopy of transversally elliptic symbols: $\operatorname{Thom}_{\xi, b}\left(\mathbb{C}^{d}\right)=\operatorname{Thom}_{0, b}\left(\mathbb{C}^{d}\right)$ in $\mathbf{K}_{T}\left(\mathbf{T}_{T} \mathbb{C}^{d}\right)$. We have proved that the class of the transversally elliptic symbol $\operatorname{Thom}_{\xi, b}\left(\mathbb{C}^{d}\right)$ in $\mathbf{K}_{T}\left(\mathbf{T}_{T} \mathbb{C}^{d}\right)$ does not depend of the data $\xi, b$.

Since the weights $\alpha_{j}$ lie enterely in an open halfspace of $\mathfrak{t}^{*}$, there exists a scalar product $b_{+}(\cdot, \cdot)$ on $\mathfrak{t}^{*}$ for which we have

$$
b_{+}\left(\alpha_{i}, \alpha_{j}\right)>0
$$

for all $i, j=1, \ldots, d$. Let $\mathcal{H}^{b_{+}}$be the Hamiltonian vector field of the function $\frac{-1}{2}\|\Phi\|_{b_{+}}^{2}$, and let $\eta_{\mathbb{C}^{d}}$ be the vector field on $\mathbb{C}^{d}$ generated by $\eta \in \mathfrak{t}$ such that $\left\langle\alpha_{j}, \eta\right\rangle>0$ for all $j$. A straightforward computation gives that

$$
\left(\mathcal{H}^{b+}(z), \eta_{\mathbb{C}^{d}}(z)\right)>0
$$

for all non zero $z \in \mathbb{C}^{d}$. Consider now the following familly of symbols on $\mathbb{C}^{d}$

$$
\sigma_{t}(z, v)=\operatorname{Thom}\left(\mathbb{C}^{d}\right)\left(z, v-\left(t \mathcal{H}^{b_{+}}+(1-t) \eta_{\mathbb{C}^{d}}\right)(z)\right), \quad(z, v) \in \mathbf{T} \mathbb{C}^{d} .
$$

so that $\sigma_{0}=\operatorname{Thom}^{\eta}\left(\mathbb{C}^{d}\right)$ and $\sigma_{1}=\operatorname{Thom}_{0, b_{+}}\left(\mathbb{C}^{d}\right)$. The inequality (1.117) shows that $\operatorname{Char}\left(\sigma_{t}\right) \cap \mathbf{T}_{T} \mathbb{C}^{d}=\{0\}$ for all $t \in[0,1]$. Hence $\sigma_{t}, t \in[0,1]$, defines an homotopy of transversally elliptic symbols: $\operatorname{Thom}^{\eta}\left(\mathbb{C}^{d}\right)=$ Thom $_{0, b_{+}}\left(\mathbb{C}^{d}\right)$ in $\mathbf{K}_{T}\left(\mathbf{T}_{T} \mathbb{C}^{d}\right)$.

For the remaining part of this paper we fix a scalar product on $\mathfrak{t}^{*}$, and we consider the family of transversally elliptic symbols $\operatorname{Thom}_{\xi}\left(\mathbb{C}^{d}\right), \xi \in \mathfrak{t}^{*}$ (to simplify, we do not mention the scalar product in the notation). Proposition 1.5.7 and (1.113) imply the following

Proposition 1.5.8 For every $\xi \in \mathfrak{t}^{*}, \mathcal{Q}^{T}\left(\mathbb{C}^{d}\right)$ is equal to the generalized character $\operatorname{Index}_{\mathbb{C}^{d}}^{T}\left(\operatorname{Thom}_{\xi}\left(\mathbb{C}^{d}\right)\right)$. 
Now we apply the techniques developped in Section 1.3 in order to compute the multiplicities of $\operatorname{Index}_{\mathbb{C}^{d}}^{T}\left(\operatorname{Thom}_{\xi}\left(\mathbb{C}^{d}\right)\right)$.

\subsubsection{Localization in a non-compact setting}

Like in Section 1.3.2 we start with the

Definition 1.5.9 For any $\xi \in \mathfrak{t}^{*}$ and any $T$-invariant relatively compact open subset $\mathcal{U} \subset \mathbb{C}^{d}$ we define the symbol $\operatorname{Thom}_{\xi}(\mathcal{U})$ by the relation

$$
\operatorname{Thom}_{\xi}(\mathcal{U})(z, v):=\operatorname{Thom}\left(\mathbb{C}^{d}\right)\left(z, v-\left(\mathcal{H}-\xi_{\mathbb{C}^{d}}\right)(z)\right) \quad(z, v) \in \mathbf{T} \mathcal{U} .
$$

The symbol $\operatorname{Thom}_{\xi}(\mathcal{U})$ is transversally elliptic when $\operatorname{Cr}\left(\|\Phi-\xi\|^{2}\right) \cap \partial \mathcal{U}=\emptyset$ (the couple $(\mathcal{U}, \xi)$ is called good) and we denote by

$$
R R_{\mathcal{U}}^{\xi}\left(\mathbb{C}^{d}\right) \in R^{-\infty}(T)
$$

its index. Proposition 1.3.10 is still valid here. In particular, for a good couple $(\mathcal{U}, \xi)$, we have $R R_{\mathcal{U}}^{\xi^{\prime}}\left(\mathbb{C}^{d}\right)=R R_{\mathcal{U}}^{\xi}\left(\mathbb{C}^{d}\right)$ if $\xi^{\prime}$ is close enough to $\xi$. Consider now the decomposition

$$
\operatorname{Cr}\left(\|\Phi-\xi\|^{2}\right)=\bigcup_{\gamma \in \mathcal{B}_{\xi}}\left(\mathbb{C}^{d}\right)^{\gamma} \cap \Phi^{-1}(\gamma+\xi) .
$$

Here $\mathcal{B}_{\xi} \subset \mathfrak{t}^{*}$ is finite set since $\mathbb{C}^{d}$ has a finite number of stabilizer. Since $0 \in\left(\mathbb{C}^{d}\right)^{\gamma}$ and $z \mapsto\langle\Phi(z), \gamma\rangle$ is constant on $\left(\mathbb{C}^{d}\right)^{\gamma}$, we have

$$
(\gamma+\xi, \gamma)=0
$$

for all $\gamma \in \mathcal{B}_{\xi}$.

Definition 1.5.10 For any $\xi \in \mathfrak{t}^{*}$ and $\gamma \in \mathcal{B}_{\xi}$, we denote simply by

$$
R R_{\gamma}^{\xi}\left(\mathbb{C}^{d}\right) \in R^{-\infty}(T)
$$

the generalized character $R R_{\mathcal{U}}^{\xi}\left(\mathbb{C}^{d}\right)$, where $\mathcal{U}$ is a $T$-invariant relatively compact open neighborhood of $\left(\mathbb{C}^{d}\right)^{\gamma} \cap \Phi^{-1}(\gamma+\xi)$ such that $\operatorname{Cr}\left(\|\Phi-\xi\|^{2}\right) \cap \overline{\mathcal{U}}=$ $\left(\mathbb{C}^{d}\right)^{\gamma} \cap \Phi^{-1}(\gamma+\xi)$.

Since $R R_{\mathbb{C}^{d}}^{\xi}\left(\mathbb{C}^{d}\right)$ is equal to $\mathcal{Q}^{T}\left(\mathbb{C}^{d}\right)$ (see Proposition 1.5.8), part $a$ ) of Proposition 1.3.10 insures that we have the decomposition

$$
\mathcal{Q}^{T}\left(\mathbb{C}^{d}\right)=\sum_{\gamma \in \mathcal{B}_{\xi}} R R_{\gamma}^{\xi}\left(\mathbb{C}^{d}\right)
$$

Let $\mathfrak{c} \subset \mathfrak{t}^{*}$ be a conic chamber of the cone $C(R)$, and take $\xi$ in $\mathfrak{c}$. Then $\xi$ is a regular value of the moment map $\Phi: \mathbb{C}^{d} \rightarrow \mathfrak{t}^{*}$ defined in (1.111). Let $\Omega_{\xi}$ be the symplectic structure on the orbifold $\left(\mathbb{C}^{d}\right)_{\xi}=\Phi^{-1}(\xi) / T$ that is induced 
from $\Omega$. The orbifold $\left(\mathbb{C}^{d}\right)_{\xi}$ is also equipped with a complex structure $J_{\xi}$ that is induced from the standard complex structure on $\mathbb{C}^{d}$, in such a way that the orbifold $\left.\left(\left(\mathbb{C}^{d}\right)_{\xi}, \Omega_{\xi}, J_{\xi}\right)\right)$ is a Kähler orbifold. If $\xi$ belongs to the lattice $\Lambda^{*}$, the reduced space $\left(\mathbb{C}^{d}\right)_{\xi}$ is the Kähler toric variety corresponding to the polytope $\left\{s \in\left(\mathbb{R}^{\geq 0}\right)^{d} \mid \sum s_{j} \alpha_{j}=\xi\right\}$ of $\mathbb{R}^{d}$. For every $\mu \in \Lambda$ we consider the holomorphic orbifold line bundle

$$
\mathcal{L}_{\xi, \mu}=\left(\Phi^{-1}(\xi) \times \mathbb{C}_{-\mu}\right) / T
$$

on $\left(\mathbb{C}^{d}\right)_{\xi}$.

Definition 1.5.11 The periodic polynomial $P_{\mathfrak{c}}: \Lambda^{*} \rightarrow \mathbb{Z}$ associated to the conic chamber $\mathfrak{c}$ is given by

$$
P_{\mathfrak{c}}(\mu)=R R\left(\left(\mathbb{C}^{d}\right)_{\xi}, \mathcal{L}_{\xi, \mu}\right),
$$

where the right hand side is the Riemann-Roch number associated to the holomorphic orbifold line bundle $\mathcal{L}_{\xi, \mu}$.

Another way to define the periodic polynomial $P_{\mathfrak{c}}$ is to consider the generalized character $R R_{0}^{\xi}\left(\mathbb{C}^{d}\right)$ for $\xi \in \mathfrak{c}$ : here $\gamma=0$ parametrizes the component $\Phi^{-1}(\xi)$ of $\operatorname{Cr}\left(\|\Phi-\xi\|^{2}\right)$. Following (1.46) we have

$$
R R_{0}^{\xi}\left(\mathbb{C}^{d}\right)=\sum_{\mu \in \Lambda^{*}} P_{\mathfrak{c}}(\mu) \mathbb{C}_{\mu} \quad \text { in } \quad R^{-\infty}(T) .
$$

After Lemma 1.3.16, we know that $R R_{0}^{\xi^{\prime}}\left(\mathbb{C}^{d}\right)=R R_{0}^{\xi}\left(\mathbb{C}^{d}\right)$ when $\xi, \xi^{\prime}$ are two elements of $\mathfrak{c}$ : hence the polynomial $P_{\mathfrak{c}}$ does not depend of the choice of $\xi$ in c.

\subsubsection{Proof of Theorem 1.5.1}

Consider a weight $\mu \in(\mathfrak{c}-\square(R)) \cap \Lambda^{*}$ of the form $\mu=\xi^{\prime}-\sum_{j} t_{j} \alpha_{j}$ with $\xi^{\prime} \in \mathfrak{c}$ and $t_{j} \in[0,1]$. We start with the decomposition

$$
\mathcal{Q}^{T}\left(\mathbb{C}^{d}\right)=\sum_{\gamma \in \mathcal{B}_{\xi^{\prime}}} R R_{\gamma}^{\xi^{\prime}}\left(\mathbb{C}^{d}\right)
$$

Since $N_{R}(\mu)$ and $P_{\mathfrak{c}}(\mu)$ are respectively the multiplicity of $\mathbb{C}_{\mu}$ in $\mathcal{Q}^{T}\left(\mathbb{C}^{d}\right)$ and in $R R_{0}^{\xi^{\prime}}\left(\mathbb{C}^{d}\right)$, the proof will be complete if we show that the multiplicity of $\mathbb{C}_{\mu}$ in $R R_{\gamma}^{\xi^{\prime}}\left(\mathbb{C}^{d}\right)$ is equal to zero when $\gamma \neq 0$.

Consider a non-zero element $\gamma$ in $\mathcal{B}_{\xi^{\prime}}$. For the character $R R_{\gamma}^{\xi^{\prime}}\left(\mathbb{C}^{d}\right)$ the localization (1.44) gives

$$
R R_{\gamma}^{\xi^{\prime}}\left(\mathbb{C}^{d}\right)=R R_{0}^{\xi^{\prime}+\gamma}\left(\left(\mathbb{C}^{d}\right)^{\gamma}\right) \otimes\left[\wedge_{\mathbb{C}}^{\bullet} \bar{N}\right]_{\gamma}^{-1},
$$


where $N=\sum_{\left(\alpha_{j}, \gamma\right) \neq 0} \mathbb{C}_{-\alpha_{j}}$ corresponds to the normal bundle of $\left(\mathbb{C}^{d}\right)^{\gamma}$ in $\mathbb{C}^{d}$. The inverse $\left[\wedge_{\mathbb{C}}^{\bullet} \bar{N}\right]_{\gamma}^{-1}$ is equal to $(-1)^{l} \mathbb{C}_{\delta(\gamma)} \otimes S^{\bullet}\left(N_{\mathbb{C}}^{+, \gamma}\right)$ where

$$
\delta(\gamma)=-\sum_{\left(\alpha_{j}, \gamma\right)<0} \alpha_{j} .
$$

Since $\gamma$ acts trivially on $\left(\mathbb{C}^{d}\right)^{\gamma}$ all the weights $\mu^{\prime} \in \Lambda^{*}$ that appear in $R R_{0}^{\xi^{\prime}+\gamma}\left(\left(\mathbb{C}^{d}\right)^{\gamma}\right)$ satisfy $\left(\mu^{\prime}, \gamma\right)=0$. Since the weights of $N_{\mathbb{C}}^{+, \gamma}$ are polarized by $\gamma$, we see from (1.121) that all the weights $\mu^{\prime} \in \Lambda^{*}$ that appear in $R R_{\gamma}^{\xi^{\prime}}\left(\mathbb{C}^{d}\right)$ must satisfy

$$
\left(\mu^{\prime}, \gamma\right) \geq(\delta(\gamma), \gamma)
$$

Consider now the weight $\mu=\xi^{\prime}-\sum_{j} t_{j} \alpha_{j}$. Since $\xi^{\prime} \in \mathfrak{c}$, the equality (1.118) implies $\left(\xi^{\prime}, \gamma\right)<0$ and then

$$
(\mu, \gamma)=\underbrace{\left(\xi^{\prime}, \gamma\right)}_{<0}+\underbrace{\sum_{\left(\alpha_{j}, \gamma\right)>0}-t_{j}\left(\alpha_{j}, \gamma\right)}_{\leq 0}-\sum_{\left(\alpha_{j}, \gamma\right)<0} t_{j}\left(\alpha_{j}, \gamma\right)<-\sum_{\left(\alpha_{j}, \gamma\right)<0}\left(\alpha_{j}, \gamma\right) .
$$

So we have proved that $(\mu, \gamma)<(\delta(\gamma), \gamma)$, hence the multiplicity of $\mathbb{C}_{\mu}$ in $R R_{\gamma}^{\xi^{\prime}}\left(\mathbb{C}^{d}\right)$ is equal to zero.

\subsubsection{Proof of Theorem 1.5.2}

Let $\mathfrak{c}_{ \pm}$be two adjacent conic chambers separated by the hyperplane $\Delta=\{\xi \in$ $\left.\mathfrak{t}^{*} \mid\langle\xi, \beta\rangle=0\right\}$. Here $\beta$ is pointing out $\mathfrak{c}_{-}$.

We consider two points $\xi_{ \pm} \in \mathfrak{c}_{ \pm}$such that $\xi=\frac{1}{2}\left(\xi^{+}+\xi^{-}\right) \in \Delta$ belongs to the conic chanber $\mathfrak{c}^{\prime}$. We suppose also that the orthogonal projection of $\xi_{ \pm}$on $\Delta$ are equal to $\xi$. We know that $P_{\mathfrak{c}_{+}}(\mu)-P_{\mathfrak{c}_{-}}(\mu)$ is equal to the $\mu$-mutiplicity of $R R_{0}^{\xi_{+}}\left(\mathbb{C}^{d}\right)-R R_{0}^{\xi_{-}}\left(\mathbb{C}^{d}\right)$. Proposition 1.3.23 tells us that

$$
R R_{0}^{\xi_{+}}\left(\mathbb{C}^{d}\right)-R R_{0}^{\xi_{-}}\left(\mathbb{C}^{d}\right)=R R_{\gamma}^{\xi_{-}}\left(\mathbb{C}^{d}\right)-R R_{-\gamma}^{\xi_{+}}\left(\mathbb{C}^{d}\right),
$$

where $\gamma \in \mathbb{R}^{>0} \beta$ is such that $\xi_{-}+\gamma=\xi_{+}-\gamma=\xi$. The localization (1.44) gives then

$$
R R_{\gamma}^{\xi_{-}}\left(\mathbb{C}^{d}\right)-R R_{-\gamma}^{\xi_{+}}\left(\mathbb{C}^{d}\right)=R R_{0}^{\xi}\left(\left(\mathbb{C}^{d}\right)^{\beta}\right) \otimes\left(\left[\wedge_{\mathbb{C}}^{\bullet}\right]_{\beta}^{-1}-\left[\wedge_{\mathbb{C}}^{\bullet}\right]_{-\beta}^{-1}\right) .
$$

Let $P_{\mathfrak{c}^{\prime}}: \Lambda^{*} \cap \Delta \rightarrow \mathbb{Z}$ be the periodic polynomial map which coincides with the vector partition function $N_{R \cap \Delta}$ on ${\overline{c^{\prime}}}^{\prime} \cap \Lambda^{*}$. If we work with the vector space $\left(\mathbb{C}^{d}\right)^{\beta}$ equipped with the hamiltonian action of $T / T_{\Delta},(1.46)$ gives the following equality in $R^{-\infty}\left(T / T_{\Delta}\right)$

$$
R R_{0}^{\xi}\left(\left(\mathbb{C}^{d}\right)^{\beta}\right)=\sum_{\gamma \in \Lambda^{*} \cap \Delta} P_{\mathfrak{c}^{\prime}}(\gamma) \mathbb{C}_{\gamma}
$$


A straightforward computation gives

$$
\left[\wedge_{\mathbb{C}}^{\bullet} \bar{N}\right]_{\beta}^{-1}=(-1)^{r^{-}} \sum_{\mu \in \Lambda^{*}} N_{R^{\prime}}\left(\mu+\delta^{-}\right) \mathbb{C}_{\mu}
$$

and

$$
\left[\wedge_{\mathbb{C}}^{\bullet} \bar{N}\right]_{-\beta}^{-1}=(-1)^{r^{+}} \sum_{\mu \in \Lambda^{*}} N_{-R^{\prime}}\left(\mu+\delta^{+}\right) \mathbb{C}_{\mu},
$$

where $r^{ \pm}, \delta^{ \pm}, R^{\prime}$ are defined in (1.106), (1.107) and (1.108). Since $N_{-R^{\prime}}(\mu)=$ $N_{R^{\prime}}(-\mu)$, the equations (1.124), (1.125) and (1.126) show that the right hand side of $(1.123)$ is equal to

$$
\sum_{\mu \in \Lambda^{*}} \sum_{\gamma \in \Lambda^{*} \cap \Delta} D(\mu) P_{\mathfrak{c}^{\prime}}(\gamma) \mathbb{C}_{\mu+\gamma}
$$

with $D(\mu)=(-1)^{r^{-}} N_{R^{\prime}}\left(\mu+\delta^{-}\right)-(-1)^{r^{+}} N_{R^{\prime}}\left(-\mu-\delta^{+}\right)$. Finally we have proved that $P_{\mathfrak{c}_{+}}(\mu)-P_{\mathfrak{c}_{-}}(\mu)=\sum_{\gamma \in \Lambda^{*} \cap \Delta} D(\mu-\gamma) P_{\mathfrak{c}^{\prime}}(\gamma)$.

\section{References}

1. M.F. АтгуAн, Elliptic operators and compact groups, Springer, 1974. Lecture notes in Mathematics, 401.

2. M.F. Атіуан, Convexity and commuting hamiltonians, Bull. London Math. Soc. 14, 1982, p. 1-15.

3. M.F. Atiyah, G.B. Segal, The index of elliptic operators II, Ann. Math. 87, 1968 , p. $531-545$

4. M.F. Atiyah, I.M. Singer, The index of elliptic operators I, Ann. Math. 87, 1968, p. 484-530.

5. M.F. Atiyah, I.M. Singer, The index of elliptic operators III, Ann. Math. 87, 1968, p. 546-604.

6. M.F. Atiyah, I.M. Singer, The index of elliptic operators IV, Ann. Math. 93, 1971, p. 139-141.

7. N. Berline, E. Getzler and M. Vergne, Heat kernels and Dirac operators, Grundlehren, vol. 298, Springer, Berlin, 1991.

8. N. Berline and M. Vergne, The Chern character of a transversally elliptic symbol and the equivariant index, Invent. Math. 124, 1996, p. 11-49.

9. N. Berline and M. VERGNe, L'indice équivariant des opérateurs transversalement elliptiques, Invent. Math. 124, 1996, p. 51-101.

10. S. Billey, V. Guillemin and E. Rassart, A vector partition function for the multiplicities of $\operatorname{sl}_{k}(\mathbb{C})$, J. Algebra, 278, 2004, p. 251-293.

11. A. Boysal and M. Vergne, Paradan's wall crossing formula for partition functions and Khovanski-Pukhlikov differential operator, arXiv:math, $\mathrm{CO} / 0803.2810$.

12. M. Brion and C. Procesi, Action d'un tore dans une variété projective, Operator algebras, unitary representations, enveloping algebras, and invariant theory (Paris, 1989), p. 509-539, Progr. Math., 92, Birkhuser Boston, Boston, MA, 1990. 
13. W. Dahmen and C. A.Michelli, The number of solutions to linear diophantine equations and multivariate splines, Trans. Amer. Math. Soc., 308, 1988, p. 509532.

14. C. De Concini, C. Procesi and M. Vergne, Partition function and generalized Dahmen-Micchelli spaces, arXiv:math, CO/0805.2907.

15. J. J. Duistermant and G. J. Heckman, On the variation in the cohomology in the symplectic form of the reduced phase space, Invent. Math., 69, 1982, p. 259-268; addendum, ibid., 72, 1983, p. 153-158.

16. J. J. Duistermant, The heat lefschetz fixed point formula for the Spin ${ }^{c}$-Dirac operator, Progress in Nonlinear Differential Equation and Their Applications, vol. 18, Birkhauser, Boston, 1996.

17. V. Guillemin, E. Lerman and S. Sternberg, Symplectic fibrations and multiplicity diagrams. Cambridge University Press 1996.

18. V. Guillemin and S. Sternberg, Convexity properties of the moment mapping, Invent. Math. 67, 1982, p. 491-513.

19. V. Guillemin and S. Sternberg, Geometric quantization and multiplicities of group representations, Invent. Math. 67, 1982, p. 515-538.

20. V. Guillemin and S. Sternberg, Birational euivalence in the symplectic category, Invent. Math. 97, 1989, p. 485-522.

21. V. Guillemin and S. Sternberg, Supersymmetry and equivariant de Rham theory. With an appendix containing two reprints by Henri Cartan. Mathematics Past and Present. Springer-Verlag, Berlin, 1999.

22. G. J. HeCKman, Projections of orbits and asymptotic behavior of multiplicities for compact Lie groups, $\mathrm{PhD}$ Thesis, University of Leiden, 1980.

23. T. KaWASAKI, The Riemann Roch theorem for complex V-manifolds, Osaka J. Math., 16, 1979, p. 151-157.

24. F. KIRWAN, Cohomology of quotients in symplectic and algebraic geometry, Princeton Univ. Press, Princeton, 1984.

25. B. Kostant, On convexity, the Weyl group and the Iwasawa decomposition, Annales Scientifiques de l'E. N. S., 6, 1973, p. 413-455.

26. S. Kumar and M. Vergne, Equivariant cohomology with generalized coefficients, Astérisque, 215, 1993, p. 109-204.

27. E. Meinrenken, On Riemaan-Roch formulas for multiplicities, J. Amer. Math. Soc., 9, 1996, p. 373-389.

28. E. Meinrenken, Symplectic surgery and the Spin ${ }^{\mathrm{c}}$-Dirac operator, Adv. in Math. 134, 1998, p. 240-277.

29. E. Meinrenken, S. SjamaAr, Singular reduction and quantization, Topology 38, 1999, p. 699-762.

30. P-E. PARAdan, Formules de localisation en cohomologie équivariante, Compositio Mathematica 117, 1999, p. 243-293.

31. P-E. PARADAN, The moment map and equivariant cohomology with generalized coefficient, Topology, 39, 2000, p. 401-444.

32. P-E. Paradan, Localization of the Riemann-Roch character, J. Funct. Anal. 187, 2001, p. 442-509..

33. P-E. PARADAN, Note sur les formules de saut de Guillemin-Kalkman, à paraître aux C.R.A.S.

34. R. SjamaAr, Symplectic reduction and Riemann-Roch formulas for multiplicities, Bull. Amer. Math. Soc. 33, 1996, p. 327-338.

35. B. Sturmfels, On vector partition functions, J. Combinatorial Theory $\mathbf{7 1}$, 1995, p. 302-309 
36. A. Szenes and M. Vergne, Residue formulae for vector partitions and EulerMacLaurin sums, Arxiv:math, CO/0202253.

37. M. Vergne, Multiplicity formula for geometric quantization, Part I, Part II, and Part III, Duke Math. Journal 82, 1996, p. 143-179, p 181-194, p 637-652.

38. M. Vergne, Quantification géométrique et réduction symplectique, Séminaire Bourbaki 888, 2001. 